\title{
Fractal structures in nonlinear dynamics
}

\author{
Jacobo Aguirre* \\ Departamento de Física, Universidad Rey Juan Carlos, Tulipán s/n, 28933 Móstoles, \\ Madrid, Spain and Centro de Astrobiología, CSIC-INTA, Ctra. de Ajalvir km. 4, \\ 28850 Torrejón de Ardoz, Madrid, Spain \\ Ricardo L. Viana ${ }^{\dagger}$ \\ Departamento de Física, Universidade Federal do Paraná, C. P. 19081, 81531-990, \\ Curitiba, Paraná, Brazil
}

\author{
Miguel A. F. Sanjuán ${ }^{\ddagger}$ \\ Departamento de Física, Universidad Rey Juan Carlos, Tulipán s/n, 28933 Móstoles, \\ Madrid, Spain
}

(Published 17 March 2009)

\begin{abstract}
In addition to the striking beauty inherent in their complex nature, fractals have become a fundamental ingredient of nonlinear dynamics and chaos theory since they were defined in the 1970s. Moreover, fractals have been detected in nature and in most fields of science, with even a certain influence in the arts. Fractal structures appear naturally in dynamical systems, in particular associated with the phase space. The analysis of these structures is especially useful for obtaining information about the future behavior of complex systems, since they provide fundamental knowledge about the relation between these systems and uncertainty and indeterminism. Dynamical systems are divided into two main groups: Hamiltonian and dissipative systems. The concepts of the attractor and basin of attraction are related to dissipative systems. In the case of open Hamiltonian systems, there are no attractors, but the analogous concepts of the exit and exit basin exist. Therefore basins formed by initial conditions can be computed in both Hamiltonian and dissipative systems, some of them being smooth and some fractal. This fact has fundamental consequences for predicting the future of the system. The existence of this deterministic unpredictability, usually known as final state sensitivity, is typical of chaotic systems, and makes deterministic systems become, in practice, random processes where only a probabilistic approach is possible. The main types of fractal basin, their nature, and the numerical and experimental techniques used to obtain them from both mathematical models and real phenomena are described here, with special attention to their ubiquity in different fields of physics.
\end{abstract}

DOI: 10.1103/RevModPhys.81.333

PACS number(s): 05.45.Df, 05.45.Ac, 05.45.Pq

\section{CONTENTS}

I. Introduction

A. Historical background: The history of determinism

B. Historical background: The birth of fractality and chaos theory

II. Basic Concepts

A. Basins of attraction and exit basins

B. Poincaré maps and stroboscopic maps

C. Graphical methods to plot basins

III. Fractals

A. The Cantor set

B. The Koch curve

C. The Sierpinski gasket

IV. Fractal Basin Boundaries

A. Original classification of fractal basin boundaries

1. Locally connected fractal basins

2. Locally disconnected fractal basins *aguirreaj@inta.es

†viana@fisica.ufpr.br

†miguel.sanjuan@urjc.es
B. Special kinds of fractal basin boundary 341

1. Intertwined basins 341

2. Wada basins 341

3. Riddled basins 342

4. Sporadically fractal basins $\quad 342$

C. Final state sensitivity and fractal dimension 343

1. The uncertainty method 343

2. The output function evaluation method 344

D. Nonattracting chaotic motion and invariant sets $\quad 344$

1. The chaotic saddle and its invariant manifolds 344

2. Graphical computation of the invariant sets $\quad 345$

3. Fractal dimension of the invariant sets 346

V. Fractal Basins in Physical Systems 346

A. Dissipative systems 347

1. Maps 347

a. One-dimensional maps $\quad 347$

b. Two-dimensional maps $\quad 347$

c. Higher-dimensional maps $\quad 347$

2. Flows 347

B. Conservative systems 349

1. Chaotic scattering 349

a. Artificial creation of exits in closed systems 351

b. Generic routes to chaotic scattering 352 
2. Open hydrodynamical flows

VI. Wada Basins

A. Topology of Wada basins

B. Accessible periodic orbits and basin cells

1. Accessible orbits

2. Basin cells

C. Computational conditions to verify the Wada property

VII. Wada Basins in Physical Systems

A. Dissipative systems

1. Maps

a. One-dimensional maps

b. Two-dimensional maps

2. Flows

B. Conservative examples

1. Chaotic scattering

2. Open hydrodynamic flows

VIII. Riddled Basins

A. Introduction and historical aspects

B. Basic definitions

C. A simple example

D. Scaling relations for riddling

E. A physical example

F. Riddling and synchronization of chaos

1. Synchronization of chaos

2. Local and global riddling

G. Riddledlike basins

1. Catastrophe of riddling

2. Pseudoriddling

IX. Experimental Verification of Fractal Basins

A. Riddled basins in electrical circuits

B. Fractal basins in chaotic scattering

1. Hydrodynamical flows

2. Optical systems

$\mathrm{X}$. Conclusions

Acknowledgments

References
352

355

355

357

357

357

358

359

359

359

359

360

361

361

362

363

365

365

366

367

370

370

373

373

374

375

375

377

377

377

378

378

379

381

381

381

\section{INTRODUCTION}

Fractal structures appear naturally in nonlinear dynamics, in such a way that the two concepts are deeply related. The impact of the ideas of chaos and fractals in physics and other scientific disciplines in recent years has been enormous. In particular, the analysis of fractality is especially useful to obtain information about the evolution of many dynamical systems modeling physical phenomena, since it provides fundamental information about the intrinsic uncertainty in predicting their future behavior.

Since the relation between fractality and nonlinear dynamics was established, it has been observed that fractality is ubiquitous in nature. All fields of science present complex systems in which prediction is a challenge because of the exponential separation of infinitely close initial conditions. In the context of dissipative systems, examples of fractal behavior are numerous. We note the appearance of fractal basins in a wide variety of nonlinear oscillators such as the Duffing oscillator (Aguirre and Sanjuán, 2002) or the forced damped pendulum
(Kennedy and Yorke, 1991), multispecies competition (Huisman and Weissing, 2001) and predator-prey models (Vandermeer et al., 2001), the modeling of electrical systems (Marcos et al., 2003) or brain dynamics (Gong and $\mathrm{Xu}, 2001)$.

Fractal basins are equally frequent among open Hamiltonian systems (Poon et al., 1996; Aguirre et al., 2001). One basic characteristic of these Hamiltonian systems is that orbits can escape toward infinity from a bounded region through one of several exits. Some applications are the analysis of the escape of stars from galaxies (Contopoulos et al., 1993), the evolution of hydrodynamical flows around obstacles (Sanjuán, Kennedy, Grebogi, et al., 1997; Sanjuán, Kennedy, Ott, et al., 1997; Kennedy et al., 1999), the dynamics of ions in electromagnetic traps (Horvath et al., 1998), planar Coulomb classical scattering (Varvoglis et al., 2001), the interaction between the Earth's magnetotail and the solar wind (Chen et al., 1990), plasma physics (da Silva et al., 2002), and the study of geodesics in gravitational waves (Veselý and Podolský, 2000), to cite just a few.

The plot of the fractal basins associated with a dynamical system provides a qualitative idea of the complications in the prediction of its future evolution. For this reason, we make a critical analysis of the existing graphical techniques, not only for computing fractal basins but also for calculation of the fractal invariant sets in Hamiltonian systems. Furthermore, in order to measure the fractality of a system quantitatively, we analyze the fundamental concept of the fractal dimension and the main algorithms used to calculate it. The main body of this review, though, is focused on the coherent presentation of the different types of fractal basin, such as Wada or riddled basins. The goal is to pay special attention to the nature, properties, and examples of the wide variety of fractal basins in the different fields of physics.

\section{A. Historical background: The history of determinism}

The notions of chaos and fractals have played a very important role in the idea of uncertainty in physics. Benoit Mandelbrot coined the term fractal in 1975 (Mandelbrot, 1975), taken from the Latin fractus meaning "broken" or "fractured," and the subsequent study of all manifestations of this beautiful mathematical structure of noninteger dimension has been fruitful in obtaining information about the behavior of many dynamical systems. Nonlinear dynamics and chaos theory, by introducing the challenging concept of final state sensitivity (Grebogi, McDonald, et al., 1983), revolutionized the classical concept of determinism, which affirms that every event or action is the inevitable result of preceding events and actions.

The ideas of determinism and its consequences are obviously much older than the concept of fractality, and are deeply related to the history of physics and philosophy. Ilya Prigogine (1917-2003), in his book The End of Certainties (Prigogine, 1996), affirms that 2500 years ago the pre-Socratic philosophers already dedicated long discussions to this subject. In particular, Epicurus (341 
B.C.-270 B.C.), who followed Democritus's ideas about nature and was convinced that the whole Universe was made out of small atoms, was especially puzzled by the meaning of human freedom in the deterministic world.

Most Western thinkers, such as Immanuel Kant (1724-1804), Alfred North Whitehead (1861-1947), or Martin Heidegger (1889-1976), defended the existence of freedom against an objective description of the world. But none of them found a convincing answer to what was named the dilemma of determinism by William James (1842-1910).

The development of science during the 16th and 17th centuries provided fundamental new information to this old debate. In particular, we must remark on Newton's second law relating force and acceleration, which is deterministic and reversible in time. If we know the initial conditions of a system and we know all the forces that are acting on it, we can obtain its future and past behavior without any ambiguity. Fascinated by this result, Pierre Simon de Laplace (1749-1827) wrote the following almost 200 years ago (de Laplace, 1814): "We may regard the present state of the universe as the effect of its past and the cause of its future. An intellect which at any given moment knew all of the forces that animate nature and the mutual positions of the beings that compose it, if this intellect were vast enough to submit the data to analysis, could condense into a single formula the movement of the greatest bodies of the universe and that of the lightest atom; for such an intellect nothing could be uncertain and the future just like the past would be present before its eyes."

During the 19th century, it was clear that conceptually the application of the laws of physics to predict the future was no longer considered a problem. The remaining challenge was based on two different limitations. First, deep knowledge of the initial conditions was necessary, and, second, the system could become extremely difficult to solve when many particles were involved. The latter difficulty pushed scientists to introduce concepts related to probability in the applicability of the physical laws. This gave rise to the new field of statistical mechanics. Among the founding fathers of this discipline are Ludwig Boltzmann (1844-1906), Josiah Willard Gibbs (1839-1903), and James Clerk Maxwell (18311879). It is known that the last was already aware of the problems associated with the uncertainty of fixing the initial conditions for a given dynamical system (Hunt and Yorke, 1993). Finally, during the 20th century and in recent years, much work has been done in order to provide a dynamical foundation to statistical mechanics, where chaos theory has played an important role (Dorfman, 1999; Gaspard, 2005; Pine et al., 2005; Gollub and Pine, 2006).

From a different perspective, the appearance of quantum mechanics had important consequences for determinism in physics. The Heisenberg uncertainty principle shows the impossibility of applying totally deterministic equations to the dynamics of the microscopic world, as it is not possible to know at the same time two conjugate variables of a particle (such as the position and velocity, for example). In this way, the deterministic laws become statistical laws. The interpretation of the wave function as a solution of the Schrödinger equation is incompatible with the deterministic concept of predicting the future position and velocity of the particle. As a consequence, the idea of indeterminism has been directly related to quantum mechanics, while most scientists have always analyzed classical mechanics as a totally deterministic subject. However, this is not so. In fact, the idea of the sensitive dependence on initial conditions was analyzed by Max Born (1882-1970) in a paper called "Is classical mechanics in fact deterministic?" (Born, 1955). He presented a study of the two-dimensional Lorentz gas, first proposed by Hendrik A. Lorentz (18531928) as a model for the electrical conductivity in metals (Lorentz, 1905a, 1905b, 1905c). In this model, a particle moves in a plane full of hard spheres, hitting them in such a way that a small change in the initial conditions will considerably alter the trajectory of the particle. Born concluded that the determinism traditionally related to classical mechanics is not real, as it is impossible to know with infinite precision the initial conditions of a physical experiment. Similar reflections were made by Erwin Schrödinger (1887-1961), and Richard Feynman (1918-1988) explained in his well-known book The Feynman Lectures on Physics (Feynman et al., 1963) that indeterminism does not belong exclusively to quantum mechanics, but is a basic property of many classical systems.

The debate has reached our times. Prigogine (1996) proposed solving the dilemma of determinism via the new fields of physics that confront indeterminism because of their basic nature: quantum theory at the microscopic level and chaos theory in the macroscopic world.

\section{B. Historical background: The birth of fractality and chaos theory}

There were several scientists in the 19th century who were aware of the idea of a sensitive dependence on initial conditions; however, the French mathematician Henri Poincaré (1854-1912) is universally recognized as the founding father of the idea of chaos in nonlinear systems. In a quite well-known citation (Poincaré, 1908), he wrote the following: "It may happen that small differences in the initial conditions produce very large differences in the final phenomena. A small error in the former produces an enormous error in the latter. Prediction becomes impossible, and we are faced with a phenomenon of chance."

The contributions of Poincaré to the theory of dynamical systems are immense. He coined the term bifurcation (Poincaré, 1885), and introduced fundamental concepts such as asymptotic solution, limit cycle, saddle node, focus, homoclinic point, and Poincaré map, apart from several important theorems such as the PoincaréBendixon theorem. There was a long lapse of time between the work of Poincaré and the development of chaos theory as we know it today. The general opinion 
of many scientists is that the appearance of the Planck hypothesis in 1900 and relativity theory in 1905 meant that many physicists dedicated their research efforts to these new and promising disciplines. Uncertainty appears naturally in quantum mechanics, and so there was no need to introduce chance in classical mechanics via sensitivity to initial conditions. On the other hand, it is believed that some of the ideas of Poincaré arrived too early, when there were almost no tools to exploit them in detail. We had to wait until the appearance of computers and numerical simulations, and in fact the impact of the ideas of Poincaré in physics was not fully understood until the meteorologist Edward Lorenz (1917-2008) published his famous results in the paper "Deterministic Nonperiodic Flow" (Lorenz, 1963). This paper soon became a milestone in the study of chaotic behavior in deterministic nonlinear dynamical systems.

The term chaos was introduced to the scientific community in the 1970s, when Tien-Yien Li and James A. Yorke published the famous paper "Period Three Implies Chaos" (Li and Yorke, 1975). The term had immediate success among the general public also, and many newspaper articles and books were written about the subject. At the same time, Benoit Mandelbrot coined the term fractal (Mandelbrot, 1975). Mathematical structures such as chaotic attractors or fractal basin boundaries have fractal dimensions, but fractals have turned out to be ubiquitous in nature. His books Fractals, Form, Chance, and Dimension (Mandelbrot, 1977) and The Fractal Geometry of Nature (Mandelbrot, 1982) soon became popular, stimulating much research. Since then, much work has been developed analyzing the relation between chaos and fractality, and this review points in this direction.

\section{BASIC CONCEPTS}

The basins of attraction in dissipative systems and the exit basins in Hamiltonian systems are two basic tools used to analyze the uncertainty associated with dynamical phenomena. The review will deal with these two concepts and the fractal structures associated with them. For this reason, this section, dedicated to clarifying several basic concepts, will define what basins of attraction and exit basins are, how they can be analyzed by making use of the concept of the Poincare map, and the main graphical methods currently available to plot basins computationally.

\section{A. Basins of attraction and exit basins}

Unlike Hamiltonian systems that preserve volume, dissipative systems have one or more attractors. For an exhaustive analysis of the term, we refer the reader to Ott (1981), Guckenheimer and Holmes (1983), Eckmann and Ruelle (1985), and Milnor (1985). In the present review we use diverse definitions of an attractor, depending on the case, but as an introduction to the concept, and following in part Strogatz (1994), we say an attractor is a set $A$ with the following properties
(1) A is a closed and bounded set that is invariant; that is, any trajectory that starts in A remains in A for all time, both positive and negative.

(2) A attracts an open neighborhood of initial conditions: there is an open set $\mathrm{U}$ containing $\mathrm{A}$ such that if $x(0) \in \mathrm{U}$, then the distance from $x(t)$ to $\mathrm{A}$ tends to zero as $t \rightarrow \infty$. The largest set $U$ that satisfies this condition is called the basin of attraction of the attractor $\mathrm{A}$.

(3) $\mathrm{A}$ is minimal; that is, there is no proper subset of $\mathrm{A}$ that satisfies conditions 1 and 2 .

(4) A is (Lyapunov) stable; that is, A has a nested family of closed neighborhoods $U_{i}$ that are positively invariant and their intersection is $A$.

The basin of attraction associated with attractor $A$ is the closure of the set of points that, taken as initial conditions, converge to A when time is increased.

For a given Hamiltonian system, an open Hamiltonian, or a Hamiltonian with escapes, represents the case for which a particle can escape toward infinity from a certain bounded region. Since there are no attractors for Hamiltonian systems, we cannot define basins of attraction. However, we can define an exit basin in an analogous way to the basins of attraction in a dissipative system. The exit basin associated with the exit $i$ of an open Hamiltonian system is the set of initial conditions that escape from the bounded region through exit $i$.

\section{B. Poincaré maps and stroboscopic maps}

To visualize basins of attraction, they must be plotted in one or two dimensions. But only two-dimensional maps and one-dimensional autonomous flows (where the variables are $x$ and $\dot{x}$ and do not depend explicitly on time $t$ ) have two-dimensional phase spaces. Since most dynamical systems are defined in a phase space with dimension higher than 2, it is not possible to plot the totality of their basins. While studying the three-body problem, Poincaré discovered that it is convenient to diminish the complexity of the analysis of a flow by studying the intersections (in a certain direction) of the trajectories with a fixed surface (Poincaré, 1897), and these surfaces are known as Poincaré surfaces of section. With this technique, a continuous $k$-dimensional flow becomes a $(k-1)$-dimensional map, commonly known as a Poincaré map [see Hénon and Heiles (1964)].

Sometimes, when we are working with nonautonomous flows that are time periodic (as happens with periodically forced oscillators, for example), it is more convenient to plot the orbits and basins of attraction after every period $\mathrm{T}$. This is called a stroboscopic map.

\section{Graphical methods to plot basins}

Several computational methods have been developed to plot the basins of attraction of a system. All we have to do in the simplest method is first find the attractors 
associated with the system, and then iterate a fine grid of initial conditions and plot them with different colors depending on the attractor they lead to. Consequently, there will be as many basins as attractors. Although some systems might have an arbitrarily high number of attractors, as reported by Feudel et al. (1996), most dynamical systems have a low number of attractors that can be easily found by using a trial and error method with a high number of random initial conditions. The same technique can be used for plotting the exit basins of an open Hamiltonian system. The only difference is that the computation of each trajectory must be paused when the trajectory has left the system through one of the exits, but in any case its initial condition will be plotted with a different color depending on the exit through which it escapes.

Advanced numerical methods have been developed to plot basins of attraction in a much faster and precise way, and in fact there exists a quite extensive variety of software dedicated to dealing with dynamical systems. In particular, the software DYNAMICS (Nusse and Yorke, 1997) has implemented two methods for the computation of basins of attraction; one called the basins and attractors method (BA) and the other called the basin method (BAS). They are explained in detail by Nusse and Yorke (1997). Although these methods are fairly complex, most typical dynamical systems are implemented in the software and, moreover, it can be modified sufficiently to analyze a wide range of new systems. The BA method plots both attractors and basins, and automatically finds the attractors. It is easy to use with the software, but the BA method works only for twodimensional maps and two-dimensional Poincaré return maps. Furthermore, it presents several uncertainties, but its improved version, the modified BA method (BAP), overcomes some of them. The BAS method is more difficult to use than the BA and the BAP methods, because the user must introduce some information about the basin to be plotted. Moreover, it finds basins but does not plot the attractors.

\section{FRACTALS}

Benoit Mandelbrot popularized a class of sets whose dimension is not an integer by introducing the term fractal in the 1970s (Mandelbrot, 1967, 1975, 1977, 1982). Broadly speaking, fractals are complex sets with a noninteger dimension. Furthermore, they present a statistical self-similarity at a wide range of different scales. Fractals soon became very popular among both the scientific community and the general public, not only because of their fascinating beauty, but also because they happen to be ubiquitous in nature. In one of his first works on the subject, Mandelbrot (1967) gives a fractal dimension of 1.25 for the coast of Britain, 1.15 for the border of Germany, and 1.14 for the border between Spain and Portugal. In particular, fractals are everywhere in biology, from cell colonies (Losa et al., 1992; Cross et al., 1995; Losa, 1995) or tumor surfaces (Brú et al., 1998, 2003) to many environmental data (Burrough,
1981). Even soil structures (Katz and Thompson, 1985) and the distribution of galaxies exhibit a specific kind of fractal-like behavior (Gaite et al., 1999; Gaite and Manrubia, 2002; Jones et al., 2004). This general interest has given rise to the appearance of an extensive literature on the subject [see Peitgen and Richter (1986); Peitgen et al. (1992); Barnsley (1993); Bunde and Havlin (1994); Frame and Mandelbrot (2002); to cite just a few].

Several fractals can be built by following very simple rules. We sketch in this section a few of them, paying special attention to Cantor sets because of the fundamental role they play in nonlinear dynamics.

\section{A. The Cantor set}

There is a whole family of fractals, the Cantor sets, that appears frequently in nonlinear dynamics. The set is named after the 19th century mathematician Georg Cantor (1845-1918) (Cantor, 1881). Nonetheless, it was a 19th century geometry professor from Oxford University, Henry Smith (1826-1883), who created it. A Cantor set is closed and consists of an uncountable number of points, each of which is a limit point. Furthermore, there are no connected subsets, except, trivially points. Any Cantor set in the real line can be constructed as follows. Start with a closed bounded interval $I_{1}$. Remove an open interval $O_{1}$ from inside it. Whenever an open interval is removed from another interval, we require that it must divide that interval into two subintervals, each of which contains more than one point. Iteratively, remove an open interval from each remaining interval in such a way that the length of the largest interval remaining at any step shrinks to zero as the process proceeds. The limiting set (consisting of the points that have not been removed) is a Cantor set. A special case is often mentioned. At each step, remove the middle third of every interval. The resulting Cantor set is called the middlethird Cantor set. Before reviewing its main properties, we must introduce the following concept that will be used throughout: A set of points in a line has zero Lebesgue measure if it can be covered with open intervals the sum of whose lengths is arbitrarily small.

The middle-third Cantor set has a noninteger dimension of $\ln 2 / \ln 3=0.631$. Furthermore, it has zero Lebesgue measure, and the proof is that the total length of the remaining interval is $(2 / 3)^{n}$ after $n$ steps of construction, and this quantity tends to zero as $n$ grows. Obviously, all end points of each interval that is removed belong to the Cantor set, but there are many other points in the unit interval that belong to the Cantor set. In fact, it is an uncountable set; this can be proved by showing that it consists of all numbers in the interval [0, 1] that can be represented in base 3 using only the digits 0 and 2 (Kennedy and Yorke, 1991; Alligood et al., 2000). Furthermore, there is a one-to-one correspondence between this Cantor set and all points in the unit interval, although the measure of the Cantor set is zero and the measure of the unit interval is 1 (see Alligood et al., 2000, and Ott, 2002, for more details). 
The middle-third Cantor set and other similar sets are common in nonlinear dynamics. We will return to them throughout the review. For example, the appearance of Cantor sets is typical in one-dimensional maps where the orbit can escape to infinity. We define the tent map as follows:

$$
T(x)=m(1-|x|)-1 .
$$

For $m>2$, almost all initial points $x_{0}$ yield trajectories of $x_{n+1}=T\left(x_{n}\right)$ that go to $-\infty$ as $n \rightarrow \infty$. If $x_{0}<-1$, then $x_{n}$ decreases monotonically and tends to $-\infty$. If $x_{0}>1$, then $x_{1}<-1$ so $x_{n} \rightarrow-\infty$. Therefore we focus our attention on the interval $J=[-1,1]$. Any trajectory that leaves $J$ must go to $-\infty$. The orbits that never escape from this bounded region constitute a Cantor set of zero Lebesgue measure. In fact, it is easy to see that, when the slope $m=3$, this fractal set coincides with the middlethird set just presented.

There is a special kind of Cantor set that does not have zero Lebesgue measure. These are the sets in which the Lebesgue measure of the union of the infinite subintervals removed from the unit interval to construct them is not equal to 1 . As a simple example, start with the unit interval, and remove in each iteration $k$ the middle subinterval of length $1 / 4^{k}$ from every remaining interval. The limiting set after infinite iterations is a fractal set of length 1/2. Farmer (1985) coined the term fat fractals for this kind of Cantor set, and Grebogi, Mcdonald, et al. (1985) defined these fat fractals as sets that lie in an $N$-dimensional Euclidean space that, for every point in the set and every $\epsilon>0$, an $\epsilon$ ball centered at that point contains a nonzero Lebesgue measure of points in the set, as well as a nonzero volume of points outside the set. Other important contributions to fat fractals are by Umberger and Farmer (1985); Gwinn and Westervelt (1986); Hanson (1987); Eykholt and Umberger (1988).

\section{B. The Koch curve}

This is a good example of how simple rules can give rise to self-similar fractal structures. The Koch curve is probably the best-known fractal curve. To construct it, start with a horizontal segment. Now, place on its middle third the base of a triangle whose side is one-third of the original segment, and erase its lower side. Repeat the action, placing one triangle of side one-third of the existing segments on the middle third of each segment of the structure, erasing the base of every triangle placed. If this algorithm is taken to the limit, the structure becomes a fractal curve. Its dimension lies between 1 and 2 $(d=\ln 4 / \ln 3=1.262)$, so that between any two points of the curve there is an infinite distance, but the curve occupies zero area (Sander, 1986).

\section{The Sierpinski gasket}

Finally, we show an example of a fractal surface. Start with a triangle, and remove the inverted triangle whose vertices are the midpoints of each side of the original triangle. Repeat this action ad infinitum with the remaining triangles. The limiting structure is a fractal surface of dimension $\ln 3 / \ln 2=1.585$.

The middle-third Cantor set, the Koch curve, and the Sierpinski gasket are absolutely self-similar, as smaller pieces of them reproduce the original set when they are magnified. However, in most fractals that appear in the context of dynamical systems, the self-similarity will not be so obvious. In these situations this term has a looser meaning, and it is used to express the idea that any magnification of a neighborhood around any point of the set never results in a smooth structure such as a single point or an isolated curve. That is, the same complex structure is maintained on all scales.

\section{FRACTAL BASIN BOUNDARIES}

When there is more than one attractor in phase space, or more than one exit in the case of an open Hamiltonian system, the basins associated with them will be separated by a basin boundary. This basin boundary can be a smooth curve or can be instead a fractal curve. A point $x$ belongs to the boundary, and is a boundary point, when every open set surrounding $x$ intersects more than one basin. Thus the basin boundary is defined by the set of boundary points. Furthermore, it is an invariant set, i.e., if $x$ belongs to the basin boundary, every subsequent image of $x$ will belong to the basin boundary.

Fractal basin boundaries have essential consequences in the lack of predictability associated with a dynamical system (Grebogi, McDonald, et al., 1983). Furthermore, they are responsible for various nonlinear phenomena, such as the destruction of chaotic attractors when they reach the fractal boundary (in what are usually named crises) (Grebogi et al., 1982, 1983a), and the appearance of long chaotic transients for values of the parameter beyond the crisis point (Grebogi et al., 1983b).

We start this section by analyzing the original classification of fractal basin boundaries exposed by McDonald et al. (1985), where these structures were divided into three main groups: locally disconnected, locally connected but not quasicircles, and quasicircles. Locally disconnected fractal basin boundaries have turned out to be much more common in physical systems, and for that reason we briefly describe the main properties of the three main types of basins that show these boundaries: intertwined, Wada, and riddled basins. (Wada and riddled basins appear so frequently in recent literature that we have dedicated Secs. VI-VIII to study them in detail.) A special type of basin with a locally connected boundary will also be addressed: the sporadically fractal basin (as is typical in the literature, we call fractal basins those basins whose boundaries are fractal). The fundamental concept of final state sensitivity and how we can measure it by making use of the fractal dimension will be addressed afterward. The section concludes by presenting the idea of nonattracting chaotic motion and a thorough analysis of the three fractal invariant sets related to it: the chaotic saddle and its invariant manifolds. Special attention will be paid to the graphical computa- 
tion and fractal dimension of these important invariant sets.

\section{A. Original classification of fractal basin boundaries}

Several types of fractal basins appear in nonlinear dynamics. Some fractal basin boundaries are continuous curves (although nondifferentiable at an uncountable number of points), but most that have been studied in the literature are discontinuous curves. A detailed protoanalysis of the classification of fractal basin boundaries and their main properties has been given by McDonald et al. (1985), where they are classified in three main groups: locally disconnected, locally connected but not quasicircles, and quasicircles.

Several concepts should be presented now, before defining these three types of fractal basin boundary.

- A closed set A is disconnected if it can be written as the union of two disjoint, nonempty closed sets. It will be connected if this is not possible.

- A set is locally connected if, given any point $\eta$ in the set and any sufficiently small $\varepsilon$, then there exists a $\delta(\varepsilon, \eta)<\varepsilon$ with the following property $\mathrm{P}$ : For every point $\xi$ in the set satisfying $|\eta-\xi| \leqslant \delta(\varepsilon, \eta)$, there is a connected subset of the original set containing $\eta$ and $\xi$ and lying wholly in the $\varepsilon$ ball centered at the point $\eta$. A set is locally disconnected if the property $\mathrm{P}$ is not satisfied for every point $\eta$ in the set.

- A set is a quasicircle if the property $\mathrm{P}$ is satisfied with $\delta=\kappa \varepsilon$ for some constant $\kappa$ independent of the point $\eta$.

From these definitions we can see that boundaries that are continuous curves or surfaces will be locally connected. On the other hand, Cantor sets are locally disconnected, because their largest connected subset is a single point, but there are infinite points in any neighborhood of any of its points.

\section{Locally connected fractal basins}

The locally connected fractal basins studied by McDonald et al. (1985) are continuous curves, although they are nondifferentiable at any point of the boundary. [It was shown in 1999 that continuous boundaries can be differentiable everywhere but in a zero Lebesgue measure set of points (Hunt et al., 1999). These basins were named sporadically fractal, and will be studied in detail later in this section.]

The main properties of locally connected basin boundaries of analytic maps with a single complex variable were studied early in the 20th century (Julia, 1918; Fatou, 1919, 1920). However, the problem had challenged the scientific community even earlier, as Cayley (1879) had already worked on determining the basins of attraction of the cubic roots of unity. [The solution came when computer work was applied to the problem one century later Curry et al., 1983; Eckmann, 1983; Blanchard, 1984; Peitgen et al., 1984; Peitgen and Richter,
1986). See Nauenberg and Schellnhuber (1989) for a complete analytical study of this problem and its physical applications.] More recent theoretical contributions to the subject are by Brolin (1966); Jakobson (1968); Sullivan (1982); Mané et al. (1983), and pioneering computational work was done by Mandelbrot (1980) and Curry et al. (1983). Analytic maps with a simple complex variable are defined as $z_{n+1}=\mathrm{F}\left(z_{n}\right)$, where $z=x+i y, x_{n+1}$ $=\operatorname{ReF}\left(x_{n}+i y_{n}\right)$, and $y_{n+1}=\operatorname{ImF}\left(x_{n}+i y_{n}\right)$. These twodimensional maps give rise to quasicircle basin boundaries that are known as Julia sets, and are the most regular types of fractal basin boundary. They are easily recognizable for their snowflake appearance, which is repeated on all scales. Mandelbrot studied the best-known type of Julia set, the ones defined as $z_{n+1}=z_{n}{ }^{2}-\mu$, where $\mu$ is a complex parameter. The Mandelbrot set, presented by Mandelbrot (1980), is defined as the set of values of $\mu$ such that the iterates of $z_{0}=0$ do not diverge to infinity. Some people claim that the Mandelbrot set is both the most complex and the most beautiful object that can be obtained from mathematics (Peitgen et al., 1992). Finally, several early key works on the understanding of Mandelbrot's bifurcation diagram should be noted (Douady and Hubbard, 1982; Douady, 1983).

However, complex analytic map are a very particular type of map, showing properties that are not generalizable to other maps or flows (for example, they do not show chaotic attractors). In this sense, they are not likely to be found in real systems, although they are relevant in the analysis of several physical phenomena, such as the study of electron motion in quasiperiodic potentials (Kohmoto et al., 1983; Ostlund et al., 1983) or the analysis of the spectral and localization properties of quantum states in hierarchical tight-binding models (Rammal, 1984).

There are other two-dimensional maps that give rise to locally connected fractal basins, but which do not satisfy the property of quasicircles. They were first presented by Grebogi et al. (1983b) and McDonald et al. (1985). Their structure is striated, and is very different from the snowflake structure of quasicircles. Furthermore, they are more likely to appear in typical dynamical systems. Grebogi et al. (1983b), Grebogi, Ott, et al. (1985), and McDonald et al. (1985) obtained analytically the continuous but nowhere-differentiable basin boundary of a two-dimensional map. The system is the following:

$$
\begin{aligned}
& x_{n+1}=\lambda_{x} x_{n}(\bmod 1), \\
& y_{n+1}=\lambda_{y} y_{n}+\cos \left(2 \pi x_{n}\right),
\end{aligned}
$$

where $\lambda_{x}$ is an integer, $\lambda_{x}>\lambda_{y}>1$, and $x$ is restricted to the unit interval. There are two attractors, one in $y=+\infty$ and the other in $y=-\infty$. The fractal basin boundary is in fact a Weierstrass curve whose equation is

$$
y=-\sum_{j=1}^{\infty} \lambda_{y}^{-j} \cos \left(2 \pi \lambda_{x}^{j-1} x\right)
$$

and its fractal dimension is 

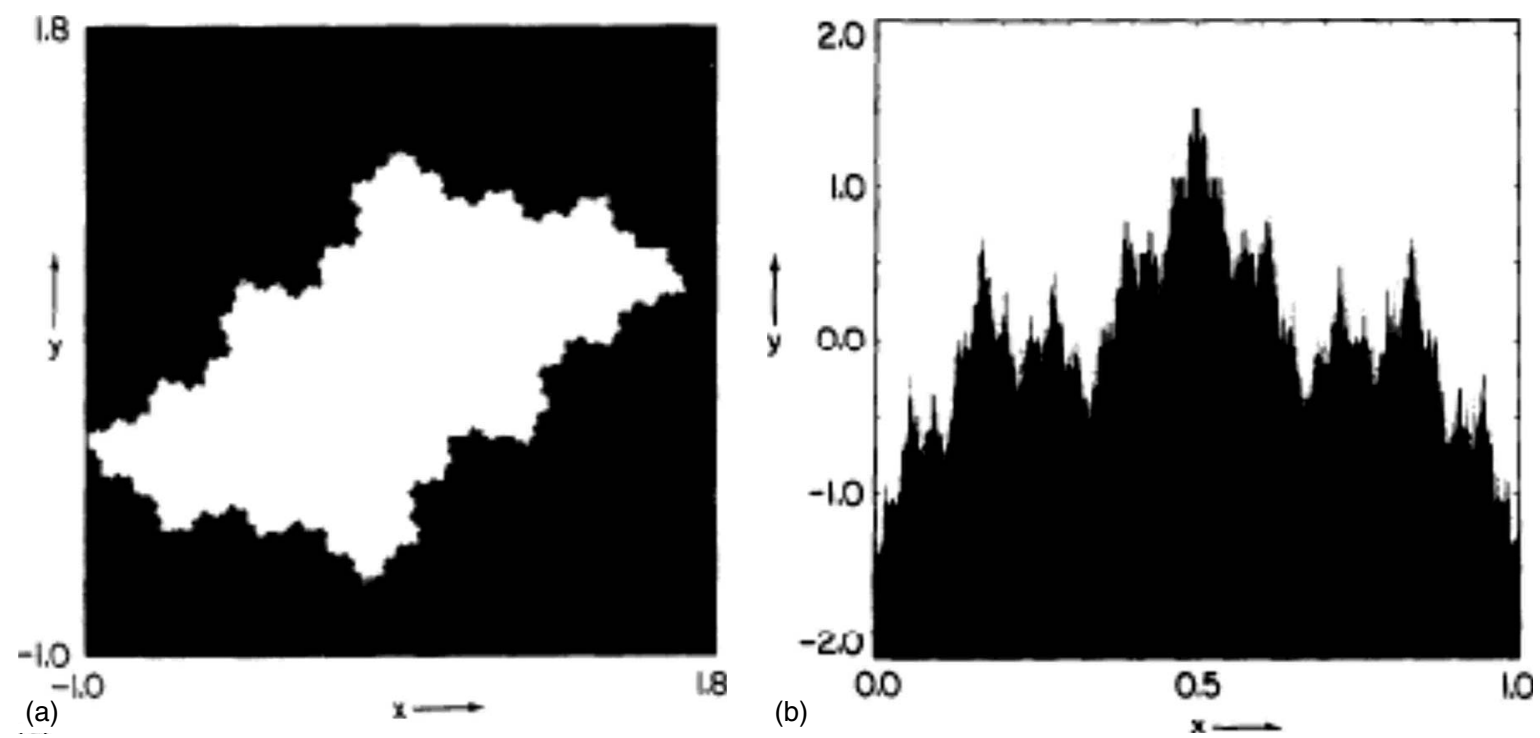

FIG. 1. Two types of locally connected fractal basin: (a) quasicircle; (b) nonquasicircle. From McDonald et al., 1985.

$$
d=2-\frac{\ln \lambda_{y}}{\ln \lambda_{x}}
$$

Figure 1 shows two examples of the locally connected fractal basins. Figure 1(a) is the basins of attraction for the analytic function $z_{n+1}=z_{n}^{2}+0.9 z_{n} \exp (2 \pi i \Omega)$, where $\Omega$ is the golden mean. These basins satisfy the conditions of quasicircles. On the other hand, Fig. 1(b) is the striated basins of attraction of the system defined by Eqs. (2) and (3) for $\lambda_{x}=3$ and $\lambda_{y}=1.5$, which are totally different from the snowflake nature of quasicircles.

All types of locally connected fractal basin boundary presented so far are very special cases of two-dimensional maps. Most dynamical systems are neither modeled by complex analytic maps nor so simple that the basin boundary can be obtained analytically. For this reason, McDonald et al. (1985) extended their analysis to more general two-dimensional maps, and found that locally connected fractal basin boundaries are indeed typical structures in dissipative systems. In particular, they obtained this result by working with nonanalytic noninvertible quadratic maps, which have been used to study a large number of physical systems, and whose properties are also present in systems of higher dimensions. They obtained two main results. First, this broad family of dynamical systems might show coexistence of attractors (and these attractors might be periodic or chaotic). Second, the basins associated with these attractors can have smooth or fractal striated boundaries.

\section{Locally disconnected fractal basins}

Before presenting the most common type of fractal basin boundary, we need to give first some important concepts.

- An equilibrium point $S$ is a saddle point if there are initial conditions whose trajectories approach $S$ when $t \rightarrow \infty$ and there are initial conditions whose trajectories approach $\mathrm{S}$ when $t \rightarrow-\infty$.
- The stable manifold of a saddle point $\mathrm{S}$ is the set $\mathrm{U}^{s}$ of initial conditions $x_{0}$ such that $x_{0}(t) \rightarrow \mathrm{S}$ when $t$ $\rightarrow \infty$. It is an invariant set.

- The unstable manifold of a saddle point $S$ is the set $\mathrm{U}^{u}$ of initial conditions $x_{0}$ such that $x_{0}(t) \rightarrow \mathrm{S}$ when $t \rightarrow-\infty$. It is an invariant set.

One main finding of Poincaré related to dynamical systems theory is that the stable and unstable manifolds of a saddle point may cross at points other than the saddle. These crossings are called homoclinic points. Poincaré discovered the existence of homoclinic points related to the three-body problem, and therefore he could show the extreme complexity of this system (Poincaré, 1897). Now, we know that every small disk centered at a homoclinic point includes infinitely many homoclinic points. Furthermore, if there is one homoclinic point, then we can find infinitely many of them. In practice, a single crossing of the stable and unstable manifolds of a saddle point necessarily means that there are infinitely many other crossings somewhere in phase space.

The basin boundary that separates two basins of attraction consists of the stable manifold of an invariant set that also belongs to the boundary (Moon and $\mathrm{Li}$, 1985). This invariant set can be a saddle point $x$ or a more complex structure. If the unstable manifold does not cross the boundary, then we say that the boundary is nonfractal. However, if the unstable manifold crosses the boundary once, there are infinitely many other crossings. Each crossing will be a homoclinic point, and will have infinitely many periodic orbits of different periods in every neighborhood of the homoclinic point. This complex situation results in a locally disconnected fractal basin boundary.

But, what is the relation between the explanation of fractal basins as a consequence of infinite intersections between stable and unstable manifolds and the one based on Cantor sets embedded in the boundary? Smale 


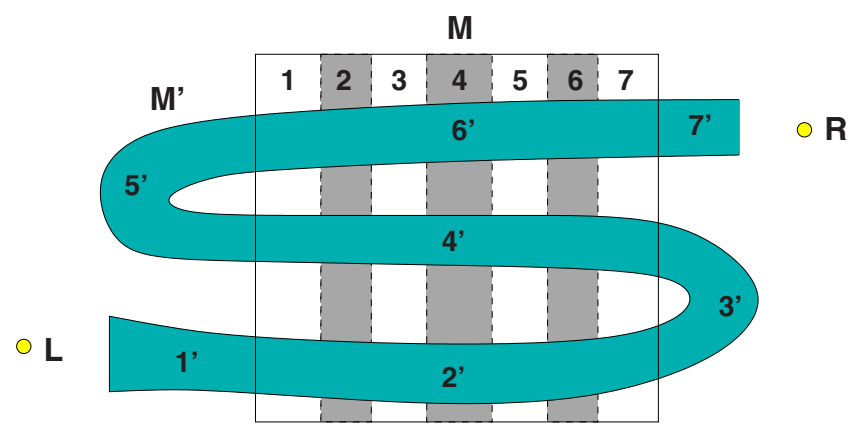

FIG. 2. (Color online) Sketch of the creation of a fractal basin boundary.

(1967) studied this problem from a geometrical point of view and proved that a homoclinic intersection in a map implies that its dynamics will be similar to that of the horseshoe map, as long as enough iterations are done. The following simple example proposed by McDonald et al. (1985) and simplified by Alligood et al. (2000) and Ott (2002) shows the natural appearance of Cantor-type fractal basins when the map (or the Poincaré section of a flow) follows dynamics similar to that of the horseshoe map.

Imagine that we have a system with two attractors $L$ and $\mathrm{R}$. We also have a rectangle $\mathrm{M}$ of initial conditions as shown in Fig. 2. Every orbit that leaves the rectangle and is mapped to the left of $M$ will fall to the left attractor, and every orbit that leaves the rectangle toward the right side will end up in the right attractor. Now suppose that the typical stretching and folding of nonlinear dynamics transforms the rectangle $M$ of initial conditions into the S-shaped structure $\mathrm{M}^{\prime}$ as shown in Fig. 2. These simple conditions are sufficient to guarantee fractal basin boundaries. It is easy to see that four vertical stripes of $\mathrm{M}$ will leave the rectangle after one iteration (numbers $1,3,5$, and 7 , which iterate into regions $1^{\prime}, 3^{\prime}, 5^{\prime}$, and $7^{\prime}$, respectively), while three will remain in $M$ transformed into horizontal stripes (numbers 2, 4, and 6). Stripes 1 and 5 will end up to the left of $M$ and therefore belong to the $L$ basin of attraction, while stripes 3 and 7 will end up to the right of $\mathrm{M}$ and therefore belong to the $\mathrm{R}$ basin of attraction. We now iterate the rectangle for a second time. The dynamics of stretching and folding in the $\mathrm{S}$ shape will again convert each remaining vertical stripe (numbers 2, 4, and 6) in seven thinner stripes, four of them escaping and three of them remaining inside $M$. If this algorithm is repeated ad infinitum, we obtain a very complex structure of alternating vertical stripes that belong to one or the other basin and which are mixed on all scales. The remaining set of vertical stripes is a zero Lebesgue measure set, and coincides with the fractal basin boundary of the system. As in the horseshoe, the invariant set of orbits that do not escape for all times is a Cantor set.

Since the work by Smale on horseshoe maps (Smale, 1967), numerous dynamical systems that contain fractal basins have been proved to present horseshoe-type dynamics. Therefore the two ways of focusing on fractality that have just been described are equivalent. On the one hand, we can say that the infinite crossings of stable and unstable manifolds of the unstable invariant sets embedded in the boundary give rise to the nonattracting invariant set that is the core of fractality. On the other hand, this invariant set has indeed the nature of a Cantor set, and can be explained geometrically, since the orbits remain in a bounded region for all iterations under a horseshoe-type map.

\section{B. Special kinds of fractal basin boundary}

Since the seminal classification of fractal basin boundaries given by McDonald et al. (1985), many other types have appeared in the literature. In the context of locally disconnected fractal basins, apart from the nominal fractal basins, a varied group of special subtypes has appeared. Despite the peculiar topology of some of them, all have been proven to be common in physical systems. The most prominent subtypes are intertwined, Wada, and riddled basins. Sporadically fractal basins are a new type of locally connected basin. We present them all in this section, from a historical point of view, and we pay special attention to their differences from nominal fractal basins. Figure 3 shows an example of each type.

\section{Intertwined basins}

Grebogi and co-workers (Grebogi, Kostelich, et al., 1987; Grebogi et al., 1988) discovered in 1987 that it is common to find basin boundaries that show different dimensions in different regions. These peculiar structures were named intertwined basins, describing situations in which every fractal region of the boundary has subregions inside where the boundary is smooth. In some sense, fractality and smoothness are intertwined on arbitrarily small scales. Their existence was shown in the paradigmatic case of the kicked double rotor (see Sec. V.A.1 for information on this high-dimensional map). In the kicked double rotor, all regions that contain fractal boundaries have a fractal dimension of 1.9, but we can always find smooth subregions of dimension 1. (Note that the dimension of the union of two sets is equal to the higher value of the two dimensions.)

\section{Wada basins}

Later on, in 1991, it was shown that some dynamical systems have three or more basins sharing the same boundary (Kennedy and Yorke, 1991). These basins were named Wada basins. Typical fractal basins might have three or more basins, but they usually present a different boundary separating each pair of basins. However, a basin B satisfies the Wada property if any initial condition that is on the boundary of one basin is also simultaneously on the boundary of another two or more basins. The first example of a system with this property was given by Kunizo Yoneyama (1877-1968) (Yoneyama, 1917), who attributed it to Takeo Wada (18821944), from whom it took the name. Both of them contributed to the early development of topology in Japan. 


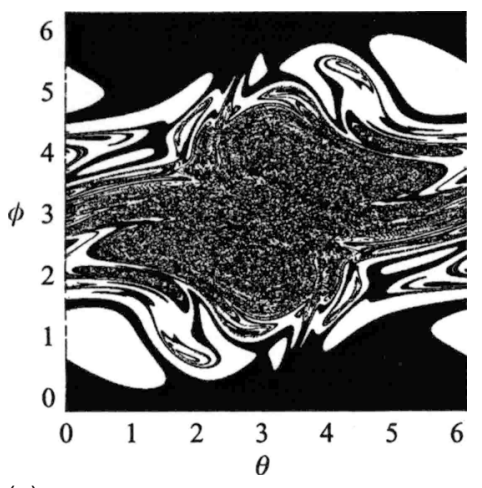

(a)

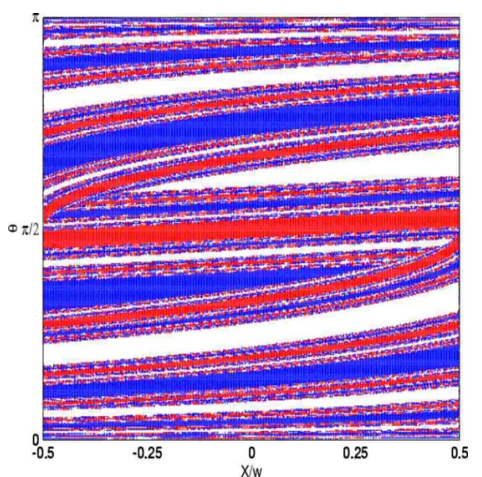

(b)

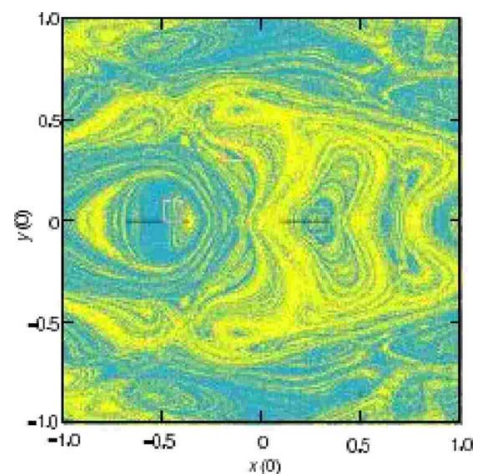

(c)

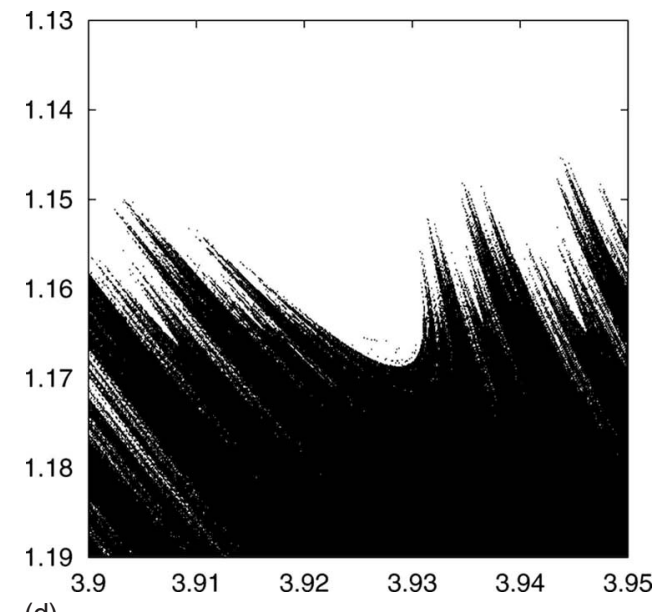

(d)

FIG. 3. (Color online) Four types of fractal basin. (a) Intertwined basins. From Grebogi, Kostelich, et al., 1987. (b) Wada basins Adapted from Aguirre and Sanjuán, 2003. (c) Riddled basins. From Sommerer, 1995. (d) Sporadically fractal basins. From Hunt et al., 1999.

The introduction of these topological ideas into nonlinear dynamics is due to Judy Kennedy and James A. Yorke (Kennedy and Yorke, 1991).

\section{Riddled basins}

Even though the Wada property shows that fractal boundaries can possess surprising topological properties, when riddled basins were presented in 1992 (Alexander et al., 1992) the scientific community received the discovery with suspicion [see Sommerer (1995) for a technical and historical introduction to the subject]. The reason is that riddled basins show that totally deterministic systems might present in practice an absolute lack of predictability. In fact, the uncertainty associated with a riddled basin is much stronger than if it were only fractal. Nowadays, this particular property related to some fractal basins has been shown in many dissipative systems. In some situations, with a special symmetry, there is an attractor whose basin of attraction has the property that every point in the basin has pieces of another attractor's basin arbitrarily nearby. Thus the entire basin is a boundary topologically, and a consequence of this definition is that riddled basins do not have any open sets inside, as is usual in other fractal basins. This type of basin is called a riddled basin, because it is "riddled" with holes of another basin. When all basins in phase space are riddled by the rest, the basins are said to be intermingled (Lai and Grebogi, 1995; Ding and Yang, 1996). This remarkable result opened the possibility of finding a degree of uncertainty unknown at the moment, leading to extensive studies, both theoretical and experimental (Ott et al., 1993; Sommerer and Ott, 1993b; Heagy et al., 1994b).

\section{Sporadically fractal basins}

Different kinds of fractal basin keep appearing in the literature, even of the locally connected type. For example, sporadically fractal basins (Hunt et al., 1999), which were qualitatively discussed by Rosa et al. (1998) and Rosa and Ott (1999) were introduced in 1999. These boundaries are continuous, smooth, and differentiable (that is, have an integer fractal dimension) at all points but a set of zero Lebesgue measure. What is striking is that, although the boundary is smooth almost everywhere, its fractal dimension is not an integer, and therefore it has limitations when trying to predict the final state of the system. This type of basin boundary needs at least dimension 2 for noninvertible maps, dimension 3 for invertible maps, and dimension 4 for flows. Furthermore, it also satisfies the intertwined property, that is, 
every fractal region of the boundary has subregions inside which the boundary is smooth.

\section{Final state sensitivity and fractal dimension}

One consequence of a dynamical system possessing fractal basins is the difficulty of making predictions, such as to which attractor the system goes, for a chosen initial condition. This is in stark contrast to the naive idea of classical determinism, where once the initial condition is fixed the evolution of the orbit is known. While every initial condition has a unique orbit in a deterministic dynamical system, the phase space might be so fractalized that serious difficulties in prediction arise if there are small uncertainties in the initial condition.

All numerical or real experiments have an unavoidable finite precision associated with the choice of initial conditions. New techniques or more developed instruments might increase this precision, but will never make an initial condition infinitely accurate (Schmick et al., 2000; Woltering and Markus, 2000b). The following explanation of the relation between uncertainty and fractal basin boundaries, adapted from Ott (2002), will help one understand the key concept of the fractal dimension. Suppose that there are several final situations for a system, and the accuracy of the technical device fixes the initial conditions with error $\epsilon$. Then there is no problem when we try to predict the future behavior of every initial condition that is further than $\epsilon$ from the basin boundary. For this reason, these initial conditions are labeled as certain. On the other hand, nothing can be predicted about the final state of initial conditions that are closer than $\epsilon$ to the boundary, and therefore they are labeled as uncertain. The fraction of uncertain initial conditions $f(\epsilon)$ for a simple smooth (i.e., integerdimensional) boundary is linearly proportional to the error associated with the initial conditions, $f(\epsilon) \propto \epsilon$. That is, if the precision of the experiment is increased by a factor $k$ (i.e., $\epsilon_{\text {new }}=\epsilon_{\text {old }} / k$ ), the fraction of uncertain initial conditions is reduced by the same factor $k$ [i.e., $f\left(\epsilon_{\text {new }}\right)$ $\left.=f\left(\epsilon_{\text {old }}\right) / k\right]$. We can say that in a nonfractal experiment the certainty is proportional to the accuracy of the measurements. However, if the boundary is fractal (i.e., it has a noninteger dimension), there exists a power-law relation between $f(\epsilon)$ and $\epsilon, f(\epsilon) \propto \epsilon^{\alpha}$, where $\alpha$ is known as the uncertainty exponent and is defined between 0 (total fractality) and 1 (smooth curve). The uncertainty dimension $D$ of the fractal set embedded in the initial conditions is obtained from $D=N-\alpha$, where $N$ is the dimension of the phase space [see Ott et al. (1985) and Ott (2002) for a proof of this statement]. Therefore the uncertainty dimension is defined in the range $D \in[N$ $-1, N]$. In summary, if there are fractal basins, a substantial increase in the precision of the experiment might lead to a very small increase in the ability to make predictions. This fact is known as final state sensitivity [see Grebogi, McDonald, et al. (1983) and McDonald et al. (1985) for a thorough study of this topic].
The fractal dimension is a common way to measure the complexity of a fractal basin boundary. As already mentioned, if it is an integer number, then the boundary is said to be nonfractal (or smooth), while if it is not an integer, the boundary is fractal. There are several definitions of the fractal dimension, all of which can give slightly different results. Some, like the correlation dimension and Kaplan-Yorke dimension, are applied to an orbit of a dynamical system. However, other definitions like the box-counting dimension and uncertainty dimension are used for general sets in the context of fractal basin boundaries. The uncertainty dimension (Grebogi, McDonald, et al., 1983; McDonald et al., 1985) has become the typical way to measure the fractality of a system, since the box-counting dimension usually requires a large number of trajectory iterates to make the error $\epsilon$ very small (Lau et al., 1991). It can be shown that the uncertainty dimension is less than or equal to the boxcounting dimension, and it has been conjectured that they coincide in typical dynamical systems (Grebogi et al., 1988). Furthermore, several classes of systems for which rigorous results have been obtained satisfy this equality between the two dimensions (Pelikan, 1985). Finally, the box-counting dimension is useless when working with fat fractals (i.e., fractal sets of positive Lebesgue measure), because the box-counting dimension of any positive measure set coincides with the total dimension of phase space, and therefore gives no information about its complex nature. In order to overcome this difficulty, the exterior dimension was proposed (Grebogi, McDonald, et al., 1985).

\section{The uncertainty method}

The typical way to compute the uncertainty dimension of a fractal set was first presented by Grebogi, McDonald, et al. (1983), and is known as the uncertainty method. The goal is to analyze how the fraction of uncertain initial conditions $f(\epsilon)$ varies with the error $\epsilon$. Typically, there are two main methods to label initial conditions as "certain" or "uncertain":

(1) The original method is based on computing the final state of the orbits. Calculate the attractor (or exit) for certain initial condition $x_{0}$. Then, compute the attractor (or exit) for the initial conditions $\left(x_{0}+\epsilon\right)$ and $\left(x_{0}-\epsilon\right)$ for a small $\epsilon$, and if all of them coincide, then this point is labeled as certain. If they do not, the point will be labeled as uncertain.

(2) The second method is based on the use of the timedelay function (Lau et al., 1991). In particular, for a fixed value of the uncertainty $\epsilon$, compute $\mid \mathbf{T}\left(x_{0}\right)$ $-\mathbf{T}\left(x_{0}+\epsilon\right) \mid$, where $\mathbf{T}\left(x_{0}\right)$ is the time-delay function defined as the time (or the number of iterations) that an orbit $x_{0}$ takes to reach an attractor in closed dissipative systems, or to escape a certain phase-space region in open Hamiltonian systems. If $\mid \mathbf{T}\left(x_{0}\right)$ $-\mathbf{T}\left(x_{0}+\epsilon\right) \mid>h$, where $h$ is a positive number, we say that $x_{0}$ is uncertain with respect to $\epsilon$ (the results are 
independent of the value of $h$, as long as $h$ is not too small). Otherwise, $x_{0}$ is certain.

Many initial conditions $x_{0}$, chosen randomly or from a fine grid, must be used to obtain a sufficiently precise estimate of the fraction of the uncertain initial conditions $f(\epsilon)$ for each $\epsilon$. If this procedure is repeated for a wide range of values of the error $\epsilon$, we obtain the dependence of $f(\epsilon)$ on $\epsilon$, which is believed to be of a power-law type. Therefore, if we plot $\ln f(\epsilon)$ vs $\ln \epsilon$, the slope will be equal to $N-D$ (with $D$ the uncertainty dimension and $N$ the dimension of the phase space). $D$ is obtained from this value, as follows:

$$
f(\epsilon) \propto \epsilon^{\alpha} \Rightarrow f(\epsilon) \propto \epsilon^{N-D} \Rightarrow \ln f(\epsilon)=(N-D) \ln \epsilon+b .
$$

It is not necessary to use a fine $N$-dimensional grid of values to calculate the uncertainty dimension. Since this can make the algorithm very slow, especially for high values of $N$, very often the computation of the uncertainty dimension can be done using a set of initial conditions of dimension $N_{0}$ (where $\left.N_{0}<N\right)$. A twodimensional Poincaré map $\left(N_{0}=2\right)$ or even a onedimensional slice $\left(N_{0}=1\right)$ of initial conditions might be enough, as the loss of precision is usually small. If we do this simplification, the fractal dimension of the basin boundary $D$ becomes

$$
D=D_{0}+N-N_{0},
$$

where $D_{0}$ is the uncertainty dimension obtained with Eq. (6), that is, the fractal dimension of the intersection between the fractal boundary and an $N_{0}$ surface (Lai et al., 2000).

\section{The output function evaluation method}

The uncertainty dimension computed with the uncertainty method is especially efficient for strongly fractal basins, that is, for basins with fractal dimensions close to the phase-space dimension. However, when fractal boundaries are almost smooth, this method converges slowly, as there are few uncertain initial conditions and most computation time is wasted. In order to overcome this difficulty, the output function evaluation (OFE) method was presented by de Moura and Grebogi (2001). The OFE method can be several orders of magnitude more efficient (i.e., faster) than the uncertainty method for boundaries with very low fractality. The fairly technical algorithm is explained in the paper, and we only comment here on its main features. It is based on computing precisely suitable output functions of the system (such as the deflection angle or the time delay). When the system shows fractal basin boundaries, the output functions also have a fractal set of singularities, and its fractal dimension is that of the basin boundary. Using a variable-sized grid, these fractal points embedded in the output function are resolved with high precision, requiring short computation time. The step size must be chosen so that the variation of the output function is kept approximately constant from one point to the next.
Once the set of fractal points has been found with a certain precision, the fractal dimension can be calculated with several methods, although the one explained by Grassberger (1986), Tél et al. (1989), and Jung and Tél (1991) is very powerful.

The OFE method is especially useful when the fractality is low, since it focuses calculations in the neighborhood of the fractal boundary. In these cases, when using the uncertainty method, the fraction of uncertain initial conditions $f(\epsilon)$ would be low unless the smallest step $\epsilon$ is very small, and in this case the computation time would be extremely long. The relation between the number of integrations $N_{\mathrm{OFE}}$ and $N_{\text {unc }}$ that must be calculated to obtain the fractal dimension of a system is

$$
N_{\mathrm{OFE}} / N_{\mathrm{unc}} \sim \epsilon^{2(N-D)-1},
$$

where $N$ is the dimension of phase space and $D \in[N$ $-1, N]$ is the fractal dimension. It is clear that when the step $\epsilon$ tends to zero, $N_{\mathrm{OFE}} / N_{\text {unc }}$ tends to $\infty$ if $D>N$ -0.5 making the uncertainty method much more efficient in strongly fractal systems, while it tends to zero if $D<N-0.5$, showing that the OFE method is clearly better in systems with low fractality.

\section{Nonattracting chaotic motion and invariant sets}

\section{The chaotic saddle and its invariant manifolds}

Nonattracting chaotic motion due to chaotic saddles embedded in the dynamics is very common, in both conservative and dissipative systems. A chaotic saddle is a closed, bounded, and nonattracting invariant set having a dense orbit (Grebogi et al., 1982, 1983a). It possesses a chaotic trajectory that never leaves the phase-space region containing the set, while almost every trajectory leaves the region after some transient time (by almost all we mean all initial conditions except for a set of Lebesgue measure zero). The dynamics on a chaotic saddle is similar to that exhibited by the two-dimensional horseshoe map possessing a hyperbolic invariant set (Wiggins, 1990).

In systems with nonattracting chaotic motion, the particles spend some time wandering erratically in a bounded region, usually named the scattering region, and after a certain time they escape toward an attractor, an exit, or infinity. The nonattracting chaotic set responsible for this chaotic motion, also known as a chaotic saddle or strange saddle, is formed by a set of orbits of zero Lebesgue measure that will never escape from the scattering region for both $t \rightarrow \infty$ or $t \rightarrow-\infty$ (Alligood $e t$ al., 2000). Its stable manifold contains the orbits that will never escape if $t \rightarrow \infty$, while the unstable manifold is formed by the orbits that will never escape if $t \rightarrow-\infty$. The orbits that constitute the chaotic set are unstable periodic orbits, of any period, or aperiodic, plus all the intersections of their stable and unstable manifolds. In fact, the number of these periodic orbits increases exponentially with their period. As the stable and unstable manifolds are invariant sets, their intersection is invari- 
ant, and therefore all orbits that start at one point belonging to the chaotic set will never leave the set.

Typically, the fractal basin boundary coincides with the stable manifold of the chaotic set embedded in the system, and consequently consists of orbits that never escape from the scattering region. If an initial point lies close to the boundary, the trajectory advances following the stable manifold towards an unstable orbit of the chaotic set, spends a long time in its vicinity, and escapes following the unstable manifold. For that reason, the trajectories that lie close to the fractal boundary are the ones that spend a longer time in the scattering region.

In hyperbolic Hamiltonian dynamical systems, the stable and unstable manifolds can intersect but are never tangent to each other. The chaotic set is the closure of intersections of a stable and an unstable manifold. Every saddle point is hyperbolic. There are no Kolmogorov-Arnold-Moser (KAM) surfaces of quasiperiodic orbits, and all the periodic orbits are unstable. Nonetheless, in nonhyperbolic Hamiltonian systems, some intersections of the stable and unstable manifolds are tangent, and KAM surfaces are mixed with chaotic regions in the phase space. The quasiperiodic orbits that belong to a KAM torus never escape from the scattering region. Therefore the KAM tori take part of the chaotic saddle, as their orbits remain inside the scattering region for both $t \rightarrow \infty$ and $t \rightarrow-\infty$. The existence or nonexistence of these surfaces brings important consequences to the dynamics of the system. In a hyperbolic system, the survival probability of a test particle in the vicinity of the chaotic saddle decays exponentially with time,

$$
P(t) \sim e^{-t / \tau},
$$

where $\tau$ is the average decay time or average transient lifetime, while stickiness to KAM surfaces should make this decay algebraic,

$$
P(t) \sim t^{-z},
$$

in nonhyperbolic systems [see Lau et al. (1991), and references therein]. The average decay time is related to the dimension of the invariant sets (Hsu et al., 1988).

\section{Graphical computation of the invariant sets}

In order to compute the chaotic invariant set of a system and its stable and unstable manifolds, the easiest way is to use the sprinkler algorithm or the sprinkle algorithm, which was first introduced by Kantz and Grassberger (1985). The main idea consists of sprinkling a large number of initial conditions from a region that contains the strange saddle. Then, every point is iterated for $t$ iterations. To pick $t$, it is sufficient to find a time when most orbits have already escaped from the vicinity of the chaotic saddle. The closer an initial point is to the stable manifold, the longer it will take to escape from the chaotic saddle, and it will follow the unstable manifold to do so. Therefore the initial points that remain in the neighborhood for a certain iteration $t$ form the stable manifold. Their $t$ iterations form the unstable manifold, and the iterations that are more or less in the middle between the first (stable manifold) and the last (unstable manifold) form the chaotic set. Furthermore, the chaotic set does not depend critically on the iteration chosen to draw it.

The sprinkler algorithm is the easiest way to compute the chaotic saddle and its invariant manifolds, but has a limited applicability. It works fine for two-dimensional systems, but not in higher dimensions. In fact, according to Sweet, Nusse, et al. (2001), this method finds points within $10^{-3}$ of the chaotic saddle, and cannot be used to calculate the Lyapunov exponents. The proper interior maxium (PIM) -triple method (Nusse and Yorke, 1989) is a much more accurate algorithm to compute the chaotic set, and shows an error of around $10^{-8}$. It is also very general, as it is applicable in high-dimensional systems and calculates the Lyapunov exponents. During the 1990s, it was generally applied to all kinds of systems with chaotic saddles, such as chaotic scattering, fluid dynamics, lasers, chaotic communications, etc. (Bleher et al., 1990; Breymann et al., 1994; Kóvacs and Wiesenfeld, 1995; Péntek, Toroczkay, et al., 1995; Bollt et al., 1997; Schwartz and Carr, 1999). A modification of the PIMtriple method used with experimental time series was presented by Jánosi and Tél (1994), where it was applied to a laser experiment. The main disadvantage of the PIM-triple method is that the chaotic set can only be one-dimensionally unstable, and many real systems [for example, the transition to turbulence in shear flows, as mentioned by Sweet, Nusse, et al. (2001)] show chaotic saddles that are $k$-dimensionally unstable, with $k>1$. The PIM-simplex method, presented by Moresco and Dowson (1999), tried to solve this question, but its applicability is limited. Finally, the first general (and simple) method to numerically calculate chaotic trajectories on chaotic saddles was presented by Sweet, Nusse, et al. (2001). It is known as the stagger-and-step method. It can be applied to maps and flows, and is applicable no matter how many unstable dimensions the system has. Furthermore, the Lyapunov exponents and thus the fractal dimension of the chaotic saddle can be calculated straightforwardly.

As an example of a typical system with nonattracting chaotic dynamics, Fig. 4 shows the invariant sets and exit basins related to the Hénon-Heiles system, a wellknown model for an axisymmetrical galaxy. It is a twodimensional dynamical system and it has three different exits for orbits over a certain threshold value of the energy of the system, called the escape energy. Due to its $2 \pi / 3$ rotation symmetry, the exits are separated by an angle $2 \pi / 3 \mathrm{rad}$, and we call them the upper exit $(y \rightarrow+\infty)$, the left exit $(y \rightarrow-\infty, x \rightarrow-\infty)$, and the right exit $(y \rightarrow-\infty, x \rightarrow+\infty)$. For a conservative Hamiltonian system, we can study its associated exit basins, which will be frequently fractal as shown by Bleher et al. (1988). (This will be studied in detail in Sec. V.B.1.) The HénonHeiles system was first studied by the astronomers Hénon and Heiles (1964), when analyzing if two or three constants of motion exist in the galactic dynamics. Their result was that a third isolating integral can be found for only a few initial conditions. It is one of the first ex- 

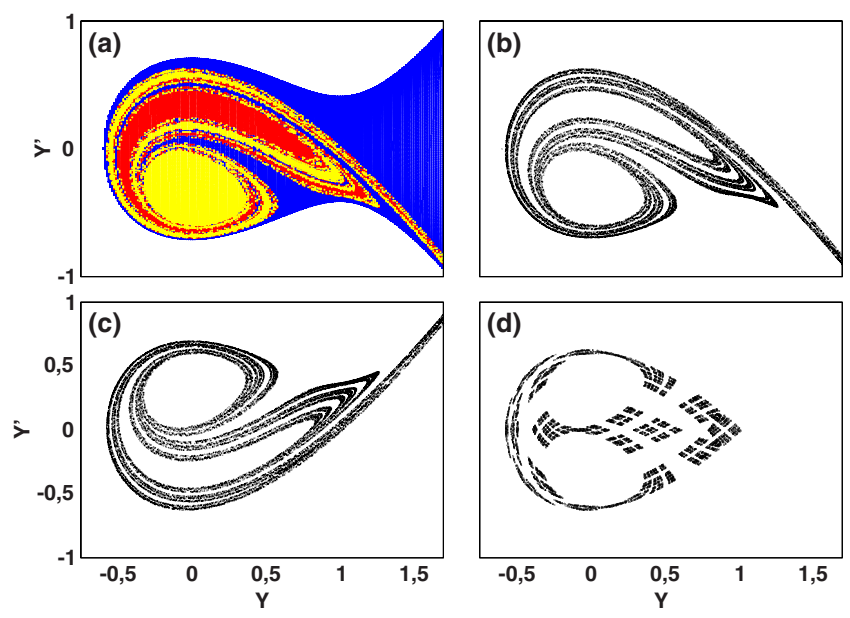

FIG. 4. (Color online) Fractal structures associated with the Hénon-Heiles system when $E=0.25$ and the system shows three exits: (a) exit basin diagram; (b) stable manifold of the chaotic invariant set; (c) unstable manifold of the chaotic invariant set; (d) chaotic invariant set. In the $(y, \dot{y})$ phase space, the upper exit is $(y \rightarrow \infty, \dot{y} \rightarrow \infty)$, and the right and left exits coincide $(y \rightarrow-\infty, \dot{y} \rightarrow-\infty)$.

amples used to show that very simple systems might possess highly complicated dynamics, and since then it has been extensively studied as a paradigm for twodimensional time-independent Hamiltonians.

The Hénon-Heiles Hamiltonian is written as

$$
H=\frac{1}{2}\left(x^{2}+y^{2}\right)+\frac{1}{2}\left(x^{2}+y^{2}\right)+x^{2} y-\frac{1}{3} y^{3} .
$$

Figure 4 shows the exit basins of the system and the invariant sets of the Hénon-Heiles system for an energy of $E=0.25$ (the escape energy for this system is $E_{e}$ $=1 / 6=0.1666 \ldots)$. The sprinkler algorithm was used, with a grid of $2000 \times 2000$ initial conditions, for the plot of the three invariant sets. As the system is defined in a threedimensional phase space, one can plot only the intersection of all these fractal sets with a Poincaré surface of section. It is clear that the stable and unstable manifolds of the chaotic set are symmetric to each other. This is reasonable, as the Hénon-Heiles potential is conservative and invariant under time-reversal transformations $(t \rightarrow-t, v \rightarrow-v)$. Typically, it is better to change the sign of every differential equation and draw the stable manifold of the new dynamical system in order to obtain the unstable manifold, because the sprinkler algorithm gives good results for the stable manifold and the strange saddle, but a very fine grid of initial conditions is needed to obtain a sufficiently dense plot of the unstable manifold. If one compares the stable manifold plotted in Fig. 4(b) with the exit basin diagram plotted in Fig. 4(a), it is clear that the stable manifold coincides with the fractal basin boundary. For this value of the energy, the chaotic set is the intersection of a stable and an unstable manifold that are never tangent, and therefore every saddle point is hyperbolic. However, the Hénon-Heiles system is nonhyperbolic for $E \in(1 / 6,0.21)$ and hyperbolic for $E>0.21$, and in the nonhyperbolic regime the exit basins show sets of initial conditions that give rise to quasiperiodic orbits that do not escape from the system (although they have the energy to do so). These orbits belong to the chaotic invariant set (Aguirre et al., 2001, 2003; Aguirre and Sanjuán, 2003).

\section{Fractal dimension of the invariant sets}

Some methods for computing the chaotic invariant set are also useful to calculate its fractal dimension (Sweet, Nusse, et al., 2001). If this is not the case, the easiest way to measure the fractality of the invariant sets is to compute the uncertainty dimension (Grebogi, McDonald, et al., 1983; McDonald et al., 1985), as explained in Sec. IV.C. As the stable manifold of the chaotic set coincides with the fractal basin boundary, the stable manifold and the system share the same fractal dimension, and therefore $D_{S}=D=D_{0}+N-N_{0}$ [see Eqs. (6) and (7) for more detail]. In Hamiltonian systems, usually the stable and unstable manifolds are symmetric, and therefore their fractal dimension is the same,

$$
D_{S}=D_{U}
$$

Since the invariant chaotic set is the intersection of its stable and unstable manifolds, its dimension satisfies $D_{C}<D_{S}=D_{U}$ and is expressed by

$$
D_{C}=D_{S}+D_{U}-N=2 D-N=2 D_{0}+N-2 N_{0} .
$$

Furthermore, and as mentioned in Sec. V.B.1, the dimensions of the three invariant sets of an open system tend to the full dimension of the phase space $N$, when the exits become arbitrarily small (de Moura and Letelier, 1999; Motter and Letelier, 2001a, 2001b; Aguirre and Sanjuán, 2003).

Finally, it is worth mentioning that the information given here on the fractal dimension of dynamical systems is true only when the $K$-dimensional phase-space region containing the chaotic set ( $K$ being an integer number) is the whole phase space (that is, $K=N$ ). If this is not the case, the invariant manifolds have dimension $D_{S}=D_{U}<K<N$, the stable manifold is not able to divide the whole phase space, and therefore we cannot talk about the existence of real fractal basin boundaries in the system.

\section{FRACTAL BASINS IN PHYSICAL SYSTEMS}

Fractality associated with nonlinear dynamics has been thoroughly studied during the last 20 years. So many systems have shown complex behavior that it is impossible to be exhaustive in the recollection of examples of fractal basin boundaries, especially in the context of dissipative systems [see Gumowsky and Mira (1980) for the first analyses of the subject]. For this reason, here we focus our attention on physical systems that can be observed in nature, although we also present a small number of mathematical systems that are especially relevant for their simplicity and clarity or for historical reasons. Furthermore, we restrict the examples to locally disconnected basin boundaries, as they are much 
more common in real systems than are locally connected ones. Examples of Wada and riddled basins are excluded, as these two subtypes will be studied later.

In particular, we divide the section into two parts: a first part dedicated to dissipative systems, and a second part dedicated to conservative systems (that is, open Hamiltonian systems). We start by studying several examples of dissipative maps, showing that even onedimensional maps might have fractal basin boundaries. In the context of dissipative flows, we pay special attention to nonlinear oscillators as examples of physical applications, and ecological models as examples of biological phenomena. Finally, chaotic scattering systems and open hydrodynamical flows are studied from the viewpoint of conservative systems.

\section{A. Dissipative systems}

1. Maps

a. One-dimensional maps

Many one-dimensional maps show complex dynamics and chaos, although in this context the systems are so simple that they usually do not represent real physical phenomena. However, it is not difficult to find a map $x_{n+1}=\mathbf{T}\left(x_{n}\right)$ where several attractors coexist and whose basins are separated by a fractal boundary. For example, Ott (2002) studied a continuous and differentiable map T with two periodic attractors and gave a detailed explanation of how the boundary that separates both complicated basins is a Cantor set.

Nevertheless, there is a straightforward way to create fractal basin boundaries in one-dimensional maps. The logistic map $[\mathbf{T}(x)=\lambda x(x-1)]$ and the tent map $[\mathbf{T}(x)$ $=m(1-|x|)-1]$ are typical examples. Depending on the parameters, they can show a wide variety of behaviors, such as periodic and chaotic attractors, or even nonattracting invariant sets. We have to find the correct value of the parameter for which there is only one period- $n$ attractor, where $n>1$. Then, the basins of the map $\mathbf{T}(x)$ applied $n$ times [that is, $\mathbf{T}^{n}(x)$ ] are separated by fractal basins (McDonald et al., 1985; Napiórowsky, 1986; Park et al., 1989). As an example, we can take the $\mathbf{T}^{3}(x)$ map, where $\mathbf{T}(x)$ is the logistic map in the period-3 regime $1.75 \lesssim \lambda \lesssim 1.79$ (Mcdonald et al., 1985). The fractal dimension for $\lambda=1.75$ is 0.97 , which suggests the extreme complexity of the boundary.

\section{b. Two-dimensional maps}

The first example in the literature of two-dimensional maps that show fractal boundaries is the following (Grebogi, McDonald, et al., 1983; McDonald et al., 1985):

$$
\begin{aligned}
& \theta_{n+1}=\theta_{n}+a \sin \left(2 \theta_{n}\right)-b \sin \left(4 \theta_{n}\right)-x_{n} \sin \left(\theta_{n}\right), \\
& x_{n+1}=-J_{0} \cos \left(\theta_{n}\right),
\end{aligned}
$$

in the regime in which there are only two fixed points as attractors (for example, $a=1.32, b=0.9$, and $J_{0}=0.3$, for which the fractal dimension is 1.8).
However, the Hénon map is the most famous twodimensional map. Many of the fundamental discoveries developed in the context of nonlinear dynamics have been made with the Hénon map before being generalized to continuous systems or experimental verification. Its mathematical expression is the following:

$$
\begin{aligned}
& x_{n+1}=a-x_{n}^{2}+b y_{n}, \\
& y_{n+1}=x_{n} .
\end{aligned}
$$

This case was first studied by Hénon (1976), and since then it has become a paradigmatic example. According to the values of the parameters $a$ and $b$, this system shows various types of fractal basin.

\section{c. Higher-dimensional maps}

Several complex dynamical systems can be transformed into high-dimensional maps, to be studied more easily. A typical example is the kicked double rotor. The experimental setup is the following. One end of a solid bar is attached to a fixed pivot, and the other end to the middle of a second solid bar. Both bars can spin around the pivots with some friction. Furthermore, a vertical and periodic impulse is applied to one end of the second bar. Grebogi, Kostelich, et al. (1987) proved that this very complex system can be analytically transformed into a four-dimensional map, where the variables are the angular positions of each bar and their angular velocities just after each kick. For certain values of the parameters, a chaotic attractor coexists with the stable fixed point that represents the situation in which both bars are vertical for all times. In this case, the basins are not only fractal, but also intertwined. This means that some parts of the basins are separated by smooth boundaries (of dimension 3), while other parts are separated by fractal basins (of dimension 3.9 in this particular case). Figure 3(a) shows the intertwined basins associated with this system.

\section{Flows}

While maps can show fractal structures even if they are one dimensional, flows need three-dimensional phase spaces to show chaos and complex dynamics.

Since the beginning of the study of nonlinear systems, many oscillators have turned out to show chaotic behavior and fractal basin boundaries for some values of their parameters. In this context, it is important to remark on the extensive work developed by J. M. T. Thompson [see Chilver (2006) for a review of his contributions to nonlinear dynamics]. For more than 20 years, Thompson analyzed the complex interplay that typically arises between chaotic transients, fractal basin boundaries, and unpredictability related to nonlinear oscillators, paying special attention to their consequences on designing technical applications [see Thompson (1992) and Thompson and Stewart (2002), and references therein].

Three nonlinear oscillators are especially ubiquitous in the literature of dynamical systems: the forced van der Pol oscillator, the forced damped pendulum, and the 


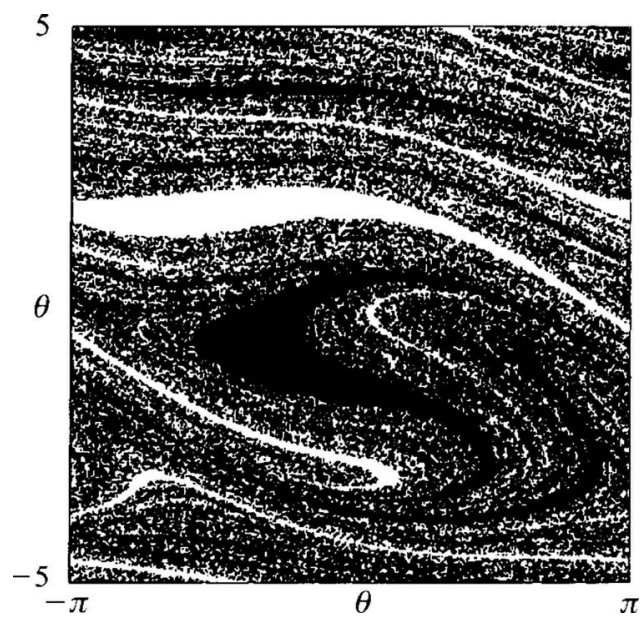

FIG. 5. Basins of attraction of the forced damped pendulum. For parameters $c=0.1, F=2.1$, and $\omega=1$ there are two different periodic attractors. From Grebogi, Ott, et al., 1987.

Duffing oscillator. The first system to be studied in the context of nonlinear dynamics is the van der Pol oscillator (van der Pol, 1920, 1927). The van der Pol oscillator was introduced in the 1920 s as an example of a simple vacuum tube oscillator circuit. If we force this oscillator with a periodic signal, we obtain the forced van der Pol oscillator, which is expressed by

$$
\ddot{x}+\left(x^{2}-1\right) \dot{x}+x=F \cos (\omega t) \text {. }
$$

Cartwright and Littlewood $(1945,1951)$ discovered the existence of nonattracting chaotic and periodic orbits in its dynamics, while Levinson (1949) studied the nature of some chaotic orbits of the system [later works showed that the orbits studied by Levinson belong to the basin boundary (Flaherty and Hoppensteadt, 1978)]. In fact, these early works on the van der Pol oscillator inspired Smale to introduce the keystone idea of the horseshoe map when developing his geometric theory of dynamical systems (Smale, 1967). But it was not until 1981 that the basin boundaries of the system were shown to have the nature of a Cantor set (Levi, 1981).

The forced damped pendulum, which is expressed mathematically as

$$
\ddot{x}+c \dot{x}+\sin (x)=F \cos (\omega t),
$$

plays a somewhat paradigmatic role for continuous dynamical systems, and for this reason appears often in all kinds of analysis in nonlinear dynamics. It represents the motion of a bob, which can oscillate or rotate, in the presence of a certain amount of friction, while it is externally forced with a periodic sinusoidal signal. It was shown to have fractal basins by Gwinn and Westervelt $(1985,1986)$, and it has been used frequently as a test system to check new results appearing in nonlinear dynamics. For example, it was the model chosen to present Wada basins by Kennedy and Yorke (1991). Figure 5 shows the numerically computed fractal basin diagram for the forced damped pendulum. For these values of the parameters, there are two periodic attractors, and therefore there are two different basins of attraction, which have been colored black and white. As the forcing of most nonlinear oscillators is $2 \pi$ periodic, in order to observe their basin diagrams it is usually more convenient to use stroboscopic maps instead of the typical Poincaré maps.

Finally, it is worth analyzing the fractal nature of the Duffing oscillator, as a paradigm of a dissipative system with applications in many fields of applied sciences and engineering (Aguirre and Sanjuán, 2002). For this reason, it has been studied by many authors [see Virgin (2000), Breban et al. (2003), and references therein]. The Duffing oscillator may be understood as a model of the one-dimensional motion of a particle of unit mass in a double-well potential, with dissipation and external periodic forcing. It is a very common physical system. An example of this kind of oscillator is a magnetoelastic mechanical system. This system consists of a beam positioned vertically between two magnets, with the top end fixed and the bottom end free to swing. In the absence of external forcing, the beam will be attracted to one of the two magnets, and will oscillate about that magnet until friction stops it. Each of the magnets creates a fixed point where the beam may come to rest and remain there in equilibrium. However, when this whole system is shaken by a periodic forcing term, the beam may jump back and forth from one magnet to the other in an apparently random manner. Depending on the intensity of the shaking term, a broad range of behaviors will be shown, from totally regular to chaotic. It was shown by Moon and Holmes (1979) that this system can be mathematically described by the Duffing equation

$$
\ddot{x}+\delta \dot{x}-\alpha x+\beta x^{3}=\gamma \cos (\omega t) .
$$

Although most work has been done on nonlinear oscillators because of the obvious technical applications, fractal basin boundaries have been found in many other fields, from economical dynamical systems (Lorenz and Nusse, 2002) to the predictability of a tossed coin (Vulović and Prange, 1986). For instance, McDonald et al. (1985) did a thorough study of the fractal basin boundaries associated with the Lorenz system (Lorenz, 1963). Although in their work McDonald et al. did not plot its basin boundaries, they used a qualitative explanation based on the concept of the broken horseshoe, presented by Kaplan and Yorke (1979), to show that for a certain value of the parameters the boundary that separates the two existing basins is created following a standard horseshoe construction, and therefore is a Cantor set. The existence of extremely long chaotic transients in the Lorenz system, which is a typical effect of fractal basins, was studied by Yorke and Yorke (1979).

Finally, it is worth remarking that extensive work has been done in the context of ecological dynamics, where the uncertainty associated with fractal basin boundaries usually represents the typical unpredictability associated with knowing the future of very sensitive ecological environments. The possibility of finding chaotic behavior associated with species competition models was already observed in the 1970s (Gilpin, 1975; May and Leonard, 1975; Smale, 1976), but several papers have focused re- 


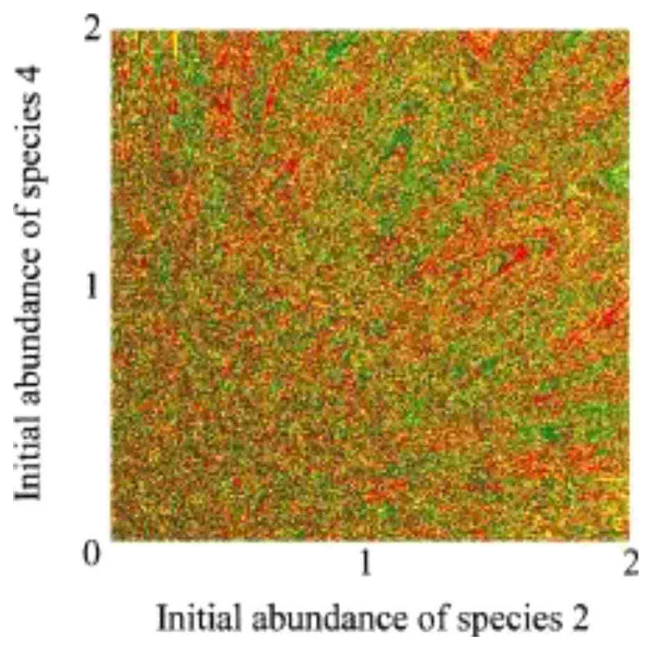

FIG. 6. (Color online) Basins of attraction of a resource competition model between eight different species, where only three are potential winners and coexistence is not possible. It is clear that the final survivor of the competition depends critically on the initial abundances of two of these species. From Huisman and Weissing, 2001.

cently on the hard-to-predict outcome of multispecies competition in ecosystems of high biodiversity. In particular, several explanations have been proposed for the observed coexistence of dozens of species of phytoplankton in aquatic ecosystems, using both dissipative systems (Huisman and Weissing, 1999) and chaotic advection systems (Károlyi et al., 2000; Scheuring et al., 2000); see Sec. V.B.2 for more information on the latter. This fact is usually called the paradox of the plankton, since by the introduction of nonequilibrium conditions and oscillatory behaviors it circumvents the principle of competitive exclusion, which predicts that at equilibrium the number of coexisting species cannot exceed the number of limiting resources. The general result is that, when the problem is analyzed using the tools of nonlinear dynamics, we observe that competition is not necessarily destructive, as competitive interactions that lead to oscillatory and chaotic behavior may support high biodiversity even with only a few limiting resources.

Despite all this literature, the appearance of fractal basin boundaries in attempts to predict the outcome of standard resource competition models is relatively recent (Huisman and Weissing, 2001; Vandermeer et al., 2001). Huisman and Weissing (2001) studied a widely used resource competition model for plankton ecology and plant ecology, and Fig. 6 shows the basin boundaries associated with the outcome-the final winner-in a competition between several species fighting for abiotic resources. The result shows a strong uncertainty associated with this type of phenomenon. The impact of these ideas in the field has been enormous, as they have opened a promising new perspective for competition models: the unpredictability associated with species interactions should not be looked for only in stochastic phenomena, as was typically accepted during the 1990s. These results explain in part the limited success of pre- dictive models of, for example, the occurrence of toxic phytoplankton species [which might be very harmful for fish and humans (Burkholder et al., 1992; Codd, 1995; Anderson and Garrison, 1997)], and suggest the development of a probabilistic focus for these systems that seem to be so unapproachable using deterministic analysis. Right now, scientists in the field are starting to assimilate the idea that "the winners of multispecies competition can be as unpredictable as a throw of a die" (Huisman and Weissing, 2001).

\section{B. Conservative systems}

\section{Chaotic scattering}

The phenomenon of chaotic scattering is associated with the dynamics of an open Hamiltonian system possessing a chaotic invariant set embedded in it; see Sec. IV.D for more information about this set and its invariant manifolds. One basic attribute of these Hamiltonian systems is the possibility of an orbit to escape from the attraction of the potential, or from a bounded region with hard walls and exits in it. Typically, a particle bounces back and forth for a certain time in a bounded area called the scattering region (in the vicinity of the chaotic invariant set), and eventually leaves it through one of the exits, escaping toward infinity (following the unstable manifold of the chaotic set). From a broad perspective, chaotic scattering consists mainly of the interaction of a particle with a system that scatters it, in such a way that the final conditions of speed and direction depend on the initial conditions [see Eckhardt (1988), Smilansky (1992), and Ott and Tél (1993) for detailed reviews of this phenomenon].

It was first shown by Bleher et al. (1988) that, when two or more escapes are possible in Hamiltonian systems, fractal boundaries typically appear. In the context of chaotic scattering, there are typically two types of system: billiards (i.e., varied configurations with hard walls) and systems defined by potentials. For this reason, Bleher et al. studied both an open chaotic billiard and a two-dimensional potential. For the first example, they chose the well-studied Sinai billiard, consisting of a twodimensional box with a central circular barrier, in which they had opened two exits in one of the walls of the external box. See Fig. 7 for a description of the billiard and the associated fractal basins. As this billiard is a two-dimensional system and the energy is conserved, the phase space is three dimensional. Therefore it is necessary to use a Poincaré surface of section to plot the exit basins. When the initial conditions that lead to each exit are plotted in different colors, the basin boundary clearly shows a fractal structure and has fractal dimension. Furthermore, these basins were one of the first examples to show the intertwined property, introduced by Grebogi, Kostelich, et al. (1987) one year before: the basin diagram shows both fractal and smooth boundaries mixed on all scales. The intertwined property has turned out to be typical of closed systems that are artificially opened in a similar way to the action of Bleher et al. with the Sinai billiard. 


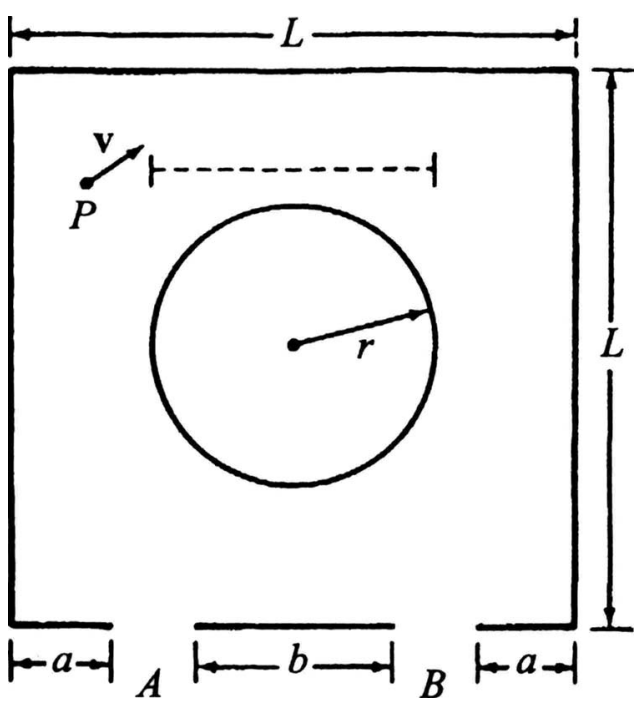

(a)

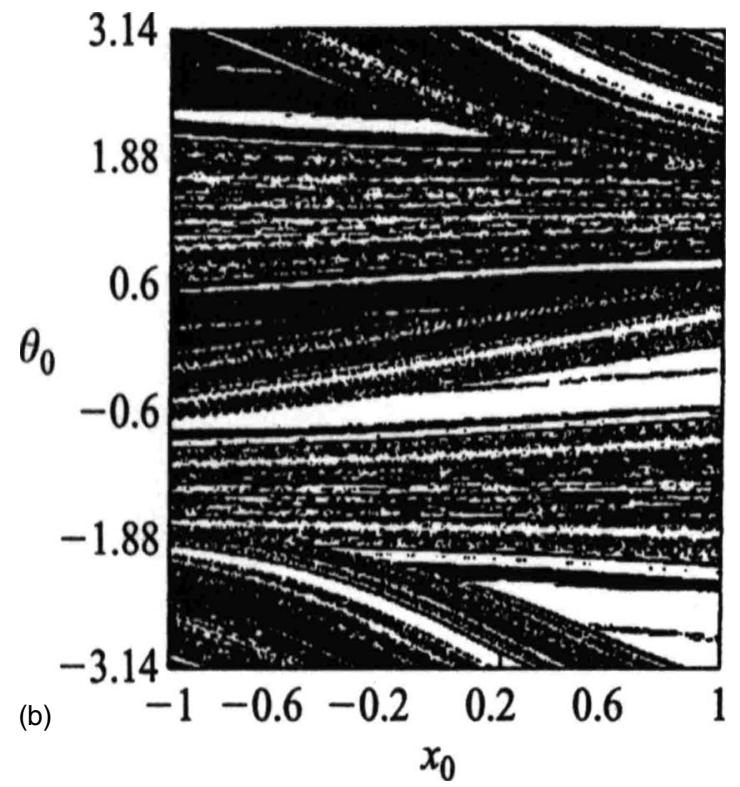

FIG. 7. The Sinai billiard was the first system to show fractal basins in the context of Hamiltonian dynamics: (a) Diagram of the system; (b) fractal exit basins associated with it. From Bleher et al., 1988.

The potential well used by Bleher et al. has two exits, and when the particle crosses them, it cannot come back into the bounded region and tends monotonically toward either $+\infty$ or $-\infty$. In this case, the whole boundary is fractal, and therefore does not satisfy the intertwined property. Two-dimensional potentials also have threedimensional phase spaces, so it is again necessary to use Poincaré sections to plot the exit basins. Many recent studies have focused on the analysis of these Hamiltonians in two dimensions, and the main reason for this interest is that they are used to model a wide range of phenomena in very different fields. Many of these applications are devoted to astrophysics, cosmology, and general relativity. For instance, much work has been dedicated to the analysis of the escape of stars from galaxies, the galaxies being idealized as two-dimensional conservative systems with an associated Hamiltonian (Contopoulos, 1990; Contopoulos and Kaufmann, 1992; Contopoulos et al., 1993) [and a review in Siopis et al. (1997)]. This work can be of great use to astronomers who need to know the decrease rate of galaxies or clusters. Furthermore, different phenomena in the solar system have received special attention: the interaction between the Earth's magnetotail and the solar wind has been analyzed by Chen et al. (1990), and in Koon et al. (2000) applied techniques taken from dynamical systems to the analysis of heteroclinic connections and resonance transitions in a planar circular restricted three-body problem. The results of the latter can be used for the design of trajectories for space missions, and cast some light on the temporal capture of Jupiter comets and asteroids [see Holmes (1990) and Simó (1999) for general reviews of the three-body problem]. In fact, in their seminal paper, Bleher et al. predicted that the existence of unpredictability due to fractal basins would be a typical feature of astrophysical systems, and they presented the orbits of some asteroids and the structure of the rings of Saturn as possible applications.

Diverse phenomena associated with general relativity can also be studied from this point of view. For instance, Steklain and Letelier (2006) applied general relativistic effects to the three-body problem formed by the Moon, the Earth, and the Sun, or the Milky Way, the M2 cluster, and a single star. Furthermore, much attention has been paid to the dynamics of light rays around black holes, where the final possible outcomes are the fall of the particle into one of the existing black holes, or its escape from the system after bouncing around the chaotic set embedded in the system (Aguirregabiria, 1997; de Moura and Letelier, 2000a, 2000c). Other works study the chaoticity of cosmological models (Motter and Letelier, 2001c), the motion of test particles in gravitational wave spacetimes (Veselý and Podolský, 2000), or the escape from multipole gravitational fields modeling galactic halos from both a Newtonian and a relativistic point of view (de Moura and Letelier, 2000b).

Exit basins do not necessarily represent escape modes of a particle from a bounded region. They can also be plotted in physical phenomena in which there are several final states. An example of this is the planar Coulomb three-body problem, in which a proton interacts with a hydrogen atom, and the final states are the excitation of the electron, the charge transfer from the hydrogen atom to the proton, and the ionization of the hydrogen atom (Varvoglis et al., 2001). Other similar cases are the classic and quantum models of the helium atom studied by Yamamoto and Kaneko (1998). Also, it is worthwhile to note the work done by Bruhn and Koch (1993) on a classical triatomic molecular configuration, since it is one of the few analytical studies of a chaotic scattering system. 
In the context of reaction dynamics, the study of the transition states during chemical reactions has led to interesting applications of chaotic scattering theory to high-dimensional systems [see Keck (1967) and Truhlar et al. (1996) for reviews on transition-state theory]. One of the main open problems in this field is the study of the complex barriers that exist between reacting and nonreacting trajectories (Wiggins et al., 2001). For instance, Waalkens et al. (2004a) used the threedimensional $\mathrm{HCN}$ isomerization problem to present a general theory for computing and analyzing the phasespace structures that appear in chemical systems with an arbitrary number of degrees of freedom. In particular, Waalkens et al. analyzed the influence of the stable and unstable manifolds of a normally hyperbolic invariant manifold (Wiggins, 1994) when studying the dividing surface in phase space that separates reactants and products. By using the computational method presented by Waalkens et al. (2004b), they showed the existence of a new type of high-dimensional chaotic saddle that might be the keystone of chaos in chemical reactions. A more mathematical analysis of the geometrical structures that regulate transition states during chemical reactions was developed by Uzer et al. (2002).

Finally, the fractal properties of Hamiltonians with more than two degrees of freedom in the context of three-dimensional billiards have been studied by Sweet and Ott (2000a, 2000b) and Sweet and Zeff (2001); see Sec. IX.B.2 for more information on the experimental realization of these systems. Continuous but nowheredifferentiable fractal basin boundaries may appear in these systems. Although this peculiar kind of basin boundary was found previously in dissipative systems (Grebogi et al., 1983b; Grebogi, Ott, and Yorke, 1985), its appearance in the context of chaotic scattering proves that it should be typical of higher-dimensional systems. On the other hand, the topological properties of the basins and invariant sets embedded in high-dimensional systems defined by potentials have been studied by Lai et al. (2000) and Kóvacs and Wiesenfeld (2001). In particular, Kóvacs and Wiesenfeld analyzed a planar atomdiatom collision with zero total angular momentum, in a regime where the scattering consists of an exchange reaction, while Lai et al. modeled a particle scattering by nonrotating diatomic molecules. Both works show that fractal and Wada basins should be typical of highdimensional systems.

\section{a. Artificial creation of exits in closed systems}

Exit basins can also be created artificially in conservative closed systems, and this strategy has proved to provide much information about the dynamical properties of the original closed system. The typical way to do this is to fix several regions in the phase space, and define the basins of each region as the set of orbits that eventually reach them. If the closed system shows chaotic behavior, fractal basins will appear associated with the open system, showing very often the coexistence of fractal and smooth basins, that is, the intertwined property [as shown by Bleher et al. (1988)]. Motter and Letelier (2001b) presented a fractal method to locate and quantify chaos in multidimensional conservative closed systems, based on the creation of artificial exits. They studied the dependence of the fractal dimension of the exit basins on the definition of the basins and the size of the exits in Motter and Letelier (2001a). They based their work on the Moura-Letelier conjecture, presented by de Moura and Letelier (1999) and qualitatively proved by Aguirre and Sanjuán (2003). This conjecture affirms that chaotic nonexit systems present invariant sets with a fractal dimension when small exits are defined, and the fractal dimension of these invariant sets tends to the phase-space dimension when the exits are arbitrarily reduced. On the other hand, if the closed system is nonchaotic, the dimension will jump discontinuously to the phase-space dimension, avoiding noninteger values when the exits are removed. [It is worthwhile to note that there are atypical examples of nonchaotic systems which exhibit fractal properties when exits are created (Troll, 1996).]

In summary, the artificial creation of exits links chaos in closed systems with fractality in open systems. Orbits wander chaotically in the scattering region of open systems before they escape from the system, while they continue their erratic evolution ad infinitum if the systems are closed.

Recently, the appearance of fractal basins when artificial exits were created in closed Hamiltonian maps has been studied by Schneider et al. (2002) and in Sanjuán et al. (2003). This tool has been shown to be fruitful in the context of plasma physics, casting some light on problems that have been unsolved for a long time, such as the control of plasma contamination due to localized heat and particle loadings on the inner tokamak wall (Engelhardt and Fenenberg, 1978), or particle anomalous diffusion in the presence of chaotic magnetic field lines (Wooton et al., 1990; Wagner and Stroh, 1993). In particular, the evolution of particles inside a tokamak before they collide with the wall can be understood as a chaotic scattering process. While the numerical integration of field line equations is always available, the use of field line mappings has proved to be very efficient (Balescu et al., 1998). Thanks to magnetic flux conservation, a field line map must be area preserving, such that the field line equations can be cast in a Hamiltonian form. In this context, da Silva et al. (2002) studied the action of a set of ergodic magnetic limiters in tokamaks in detail, with special attention to fractal structures and invariant sets. Three artificial exits were defined by dividing the tokamak wall into three different regions, and the associated basins clearly showed intertwined fractal basins. Furthermore, the fractal component of the exit basin boundary is responsible for the nonuniformity of the heat and particle loadings on the tokamak wall, proving again the direct influence of fractality in many physical phenomena. Finally, the general appearance of fractal (and also Wada) basins in the study of magnetic footprints of chaotic magnetic field lines on the tokamak wall (or in general on any obstacle placed in the chaotic 

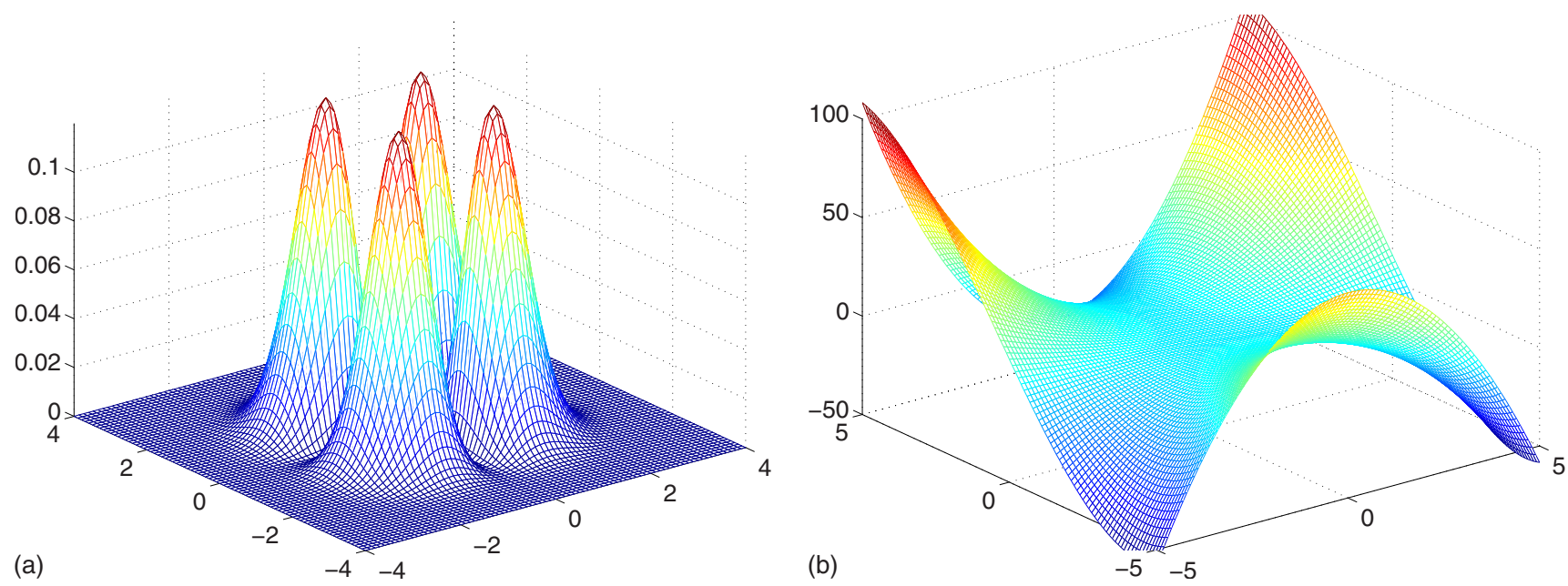

FIG. 8. (Color online) Two types of potential are responsible for the appearance of chaotic scattering via an abrupt bifurcation: (a) type A, potential with peaks studied by Bleher et al. (1990) that gives rise to fully developed chaos; (b) type B, potential analyzed by Aguirre et al. (2001) and de Moura and Letelier (1999) that gives rise to three exits when the energy is over a critical value.

region of the tokamak) was analyzed by Portela et al. (2007).

\section{b. Generic routes to chaotic scattering}

The two generic routes to chaotic scattering when a parameter is varied were studied by Bleher et al. (1990) and Ding et al. (1990). In the first case, chaotic scattering is created via a saddle-center bifurcation. The homoclinic intersection of the stable and unstable manifolds of the new saddle created after the bifurcation is responsible for the chaotic behavior. The stable center is surrounded by periodic, quasiperiodic, and chaotic orbits. The second route was shown by Bleher et al. (1990), and is based on a type of bifurcation that had not been studied before, the abrupt bifurcation to chaotic scattering. This bifurcation gives rise to fractal basins in the context of scattering situations defined by potentials, and appears in two different ways.

(1) Type-A potentials: When a certain value of the energy is crossed in the decreasing direction, some open Hamiltonian systems suffer an abrupt transition from regular to chaotic behavior. This behavior is typical of potentials with peaks (Eckhardt and Jung, 1986; Jung and Scholz, 1987; Bleher et al., 1990), and the critical energy is usually the maximum value of the potential [see Fig. 8(a) for an example]. Furthermore, this route to chaotic scattering creates fully developed chaos, which means that the dynamics is hyperbolic (that is, there are no KAM tori of quasiperiodic orbits and all periodic orbits are unstable).

(2) Type-B potentials: In this case, for energies below a certain threshold value, which is commonly called the escape energy, the orbits are bounded and the test particles cannot leave the scattering region. However, if the energy is above this threshold value, several exits appear and the orbits may escape towards infinity through any one of them (de Moura and Letelier, 1999; Aguirre et al., 2001) [see Fig. 8(b) for an example of this type of potential, the HénonHeiles system, where three exits are possible]. The fractality is maximum just over the escape energy, when the size of the exits is extremely small. When the energy increases, the exits become wider and wider and the fractal dimension decreases. In some systems, when the energy grows, the fractal dimension tends to a fixed noninteger value and therefore fractality exists for all energies, while in other systems the fractal dimension eventually reaches an integer value for a certain value of the energy and therefore the basins are smooth for all energies over this critical value. Most type-B potentials show hyperbolic and nonhyperbolic behavior depending on the value of the energy.

The chaoticity associated with both types of potential is seen when a variable of the outcoming particle (for example, the deflection angle or the escape time) is plotted versus a variable of the incoming particle (for example, the incoming angle). If the system is in the regular regime, these curves are continuous and smooth, and all orbits escape eventually from the scattering region. However, in the chaotic regime a zero Lebesgue measure set of orbits stays forever inside the bounded region, and a fractal set of singularities appears in the plottings. These orbits that never escape from the system belong to the fractal basin boundary, and coincide with the stable manifold of the invariant chaotic set embedded in the system.

\section{Open hydrodynamical flows}

Chaotic advection has recently attracted the interest of numerous scientists, and a wide variety of papers and books studying transport and mixing in two-dimensional open flows has been published (Ottino, 1989, 1990; Crisanti et al., 1991; Wiggins, 1992; Toroczkai et al., 1998; Budyansky et al., 2004; Tel et al., 2005). Coloring with 
dye certain regions of the flow and analyzing the evolution of their boundaries has turned out be a fruitful tool for understanding the dynamics of these systems (Aref et al., 1989; Kennedy and Yorke, 1997; Péntek et al., 1995a, 1995b, 1996; Péntek, Toroczkay, et al., 1995). A particle in this context is a light and small granule, and if we suppose that inertial effects are negligible, the phenomenon is called passive advection, and we name each particle a passive tracer.

Suppose that the fluid is incompressible. The basic idea of chaotic advection is that it might be possible to simplify and accelerate the computation of the evolution of a two-dimensional fluid around obstacles or vortices using two ordinary differential equations instead of solving the Navier-Stokes equations. The equations of motion of a passively advected dye particle become

$$
\begin{aligned}
& v_{x}=\partial \phi(x, y, t) / \partial y, \\
& v_{y}=-\partial \phi(x, y, t) / \partial x,
\end{aligned}
$$

where $\phi(x, y, t)$ is the stream function (which depends on the point and the time), and $v_{x}$ and $v_{y}$ are the components of the velocity field. What we need is an approximate form of the velocity field, or an approximate stream function $\phi$ that verifies that the computations are similar to what is seen when we numerically solve the Navier-Stokes equation. It is clear that the mathematical formulation of the problem becomes similar to the Hamilton equations, where the stream function $\phi$ plays the role of the Hamiltonian. In order to simplify the results, it is usually supposed that the flows are time periodic, and therefore the results can be shown in stroboscopic maps after integer multiples of the period $\mathrm{T}$.

In the beginning of the 1990s, several difficulties appeared in the literature in attempts to interpret the chaotic orbits associated with advection. It was not until 1993 (Jung et al., 1993) that the problem was first analyzed from the point of view of chaotic scattering and transient chaos. In the context of the dynamics of a flow around a cylinder, this work paid special attention to the time-delay function, the decay statistics, and the existence and nature of periodic orbits. As a new step in the same direction, and studying a similar model, Péntek et al. (1995a) addressed the importance of the chaotic invariant set embedded in the dynamics of the system, as well as its stable and unstable manifolds. The chaotic invariant set was shown to be the closure of an infinitely high number of unstable periodic orbits, and their heteroclinic and homoclinic connections, like the ones studied in other open systems.

Nowadays, it is widely accepted that the phenomenon of chaotic advection in open two-dimensional flows is identical to that of chaotic scattering in an open system. The flow is very simple and homogeneous if we go upstream or downstream (although a smooth stationary flow field would be enough), while the time dependence is restricted to a finite area called the mixing region, such as the surroundings of a rock in a river. In fact, the fluid might be either compressible or not, as long as it shows a chaotic saddle.

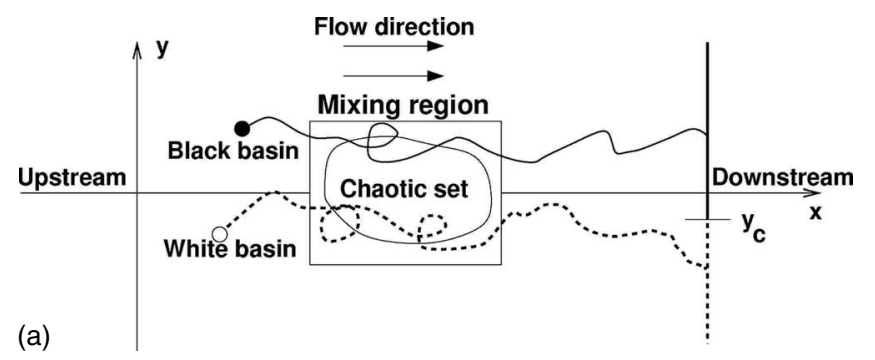

(b)

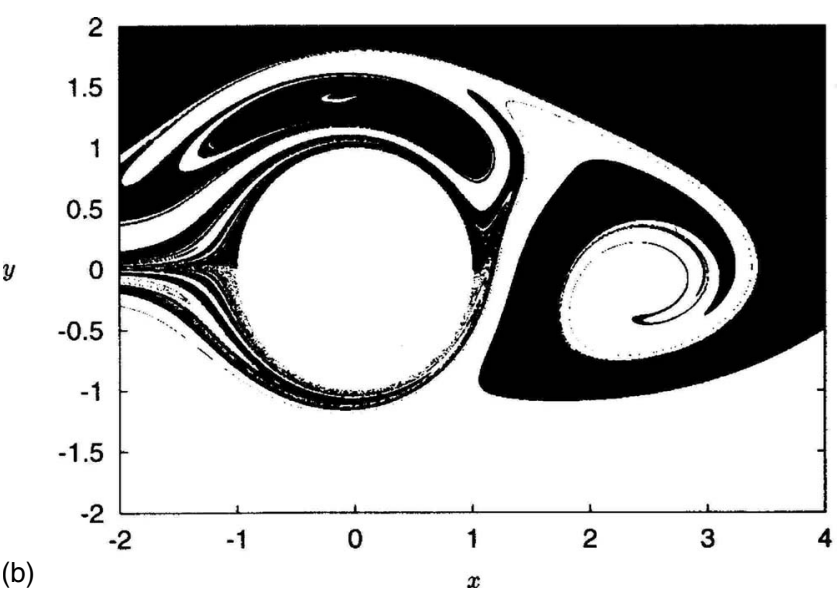

FIG. 9. Fractal basins are typical in open hydrodynamical flows. (a) Sketch of the outflow experiment for an open hydrodynamical flow around an obstacle. The diagram for the inflow experiment would place the vertical line upstream instead of downstream. (b) Fractal basins of a flow around a cylinder. Black points cross the vertical line placed at $x=6$ with positive $y$, while white points cross that line with negative $y$. The cylinder is plotted in white, too. The fractal structure is repeated after every period T. (b) from Péntek, Toroczkai, et al., 1995.

A serious difficulty must be faced when trying to plot the basin diagrams of hydrodynamical systems. The problem, of course, is that in general there are not several escapes in a flow, but a single upstream direction and a single downstream direction. How then can we define the exit basins in order to study their fractality? Péntek, Toroczkai, et al. (1995) solved the situation by presenting two possible methods: the outflow experiment and the inflow experiment.

(1) The outflow experiment is similar to what has been typically done in chaotic scattering systems where several exit modes are possible. In this case, a vertical line placed downstream (that is, after the obstacle) is divided into two segments $\left[y<y_{c}\right.$ and $y$ $>y_{c} y_{c}$ being a preselected value; see Fig. 9(a) for a schematic diagram]. In this way, two artificial exits are created, and the basin diagram is found by plotting in different colors the initial conditions that give rise to orbits that cross each segment. The boundary that separates the two existing basins is placed in the inflow region and the mixing region, and is typically fractal. Furthermore, it coincides with the stable manifold of the chaotic set embedded in the system. Figure 9(b) shows a snapshot of the numerically computed fractal basins for the movement of a fluid 
around a cylinder. Take into account that for open hydrodynamical flows the fractal basins evolve with time, being repeated in every period of the dynamics.

(2) The inflow experiment shows the opposite point of view to what has been typically seen in chaotic scattering. However, it is especially interesting because it gives rise to fractal basins that can be "physically observed" in any experiment. In some sense, it is one of the few simple real manifestations of the existence of fractal basins; see Sec. IX.B.1 for more on this subject. The key idea is to place the vertical segment in the inflow region, and divide it again into two vertical segments. Then each segment is dyed with a different color. When the system evolves with time, the colored regions approach the mixing region and start to get distorted with the typical stretching and folding phenomenon of nonlinear systems. After a long time, a stationary pattern is reached, and the stroboscopic map shows an extraordinarily complex mixture of colors. The boundaries that separated the original bands have fractalized, and can be found in the mixing region and further downstream, but not in the inflow region. Péntek, Toroczkai, et al. (1995) showed that the boundary is transported to the downstream region in a way that asymptotically accumulates on the unstable manifold of the chaotic saddle. The only necessary condition for this accumulation, though, is that the original boundaries cross the stable manifold that is placed in the inflow region.

Finally, it is important to remark that advection is of capital importance from the point of view of environmental science. In fact, dyes in liquids and pollutants in the air are spread mainly due to chaotic advection, while diffusion has a small influence in this phenomenon [see Tél and Gruiz (2006) for an analysis of the environmental significance of chaotic advection].

In summary, the chaotic behavior of passive particles inside an open flow is due to the existence of an invariant chaotic set embedded in the system, and it is known that passive tracers exhibit chaotic trajectories even for simple time-periodic cases, as long as flows are time dependent (Ottino, 1989). The particles approach the mixing region following the stable manifold of the chaotic saddle, spend some time in the vicinity of the chaotic saddle, and finally escape from the system following its unstable manifold. It is worth noting that in twodimensional flows the boundaries become a fractal of dimension between 1 and 2 (Péntek et al., 1995a, 1995b, 1996; Péntek, Toroczkai, et al., 1995), while they fill up the whole phase space if the system is completely closed (Aref and Balachandar, 1986; Chaiken, 1986; Muzzio et al., 1992).

Active chaotic advection. The results obtained for passive chaotic advection have motivated recent studies on particles that are transported by the fluid and at the same time suffer chemical or biological interactions. The growing interest of the scientific community on environ- mental challenges is making this subject one of the most promising meeting points between physics and biology. In fact, numerous ecological and industrial problems can be understood using these ideas (Metcalfe and Ottino, 1994; Ottino, Souvaliotis, and Metcalfe, 1995). While passive particles follow the fluid without any interaction between them, active particles are tracers that undergo certain changes during the evolution of the flow, although they do not affect the nature of the fluid that transports them. The goal is to cast light onto chemical or biological processes of fundamental importance, such as the population of microorganisms in the sea (Gibson and Thomas, 1995; Thomas et al., 1997), the physics of species coexistence (Károlyi et al., 2000), or ozone reactions (Edouard, Legras, Lefvre, et al., 1996; Edouard, Legras, and Zeitlin, 1996) [see Tél et al. (2005) for a review on chemical and biological activity in open flows].

Many studies have concentrated on the advection of a flow around a cylinder (Sanjuán, Kennedy, Grebogi, et al., 1997; Toroczkai et al., 1998; Kennedy et al., 1999), as this system is similar to many real situations of environmental flows, such as the motion of air around a mountain or the water in a river around a rock. The key is the long time that the tracers spend around the chaotic saddle and its unstable manifold, moving around the bounded orbits that never leave the mixing region. The particles follow the fractal structure in a way that they improve the interaction with each other for a long time. We say that the unstable manifold acts as a catalyst for the active process [see Toroczkai et al. (1998) for a study of this phenomenon].

In order to clarify the importance of chaotic advection in ecological and environmental contexts, we pay special attention to a model of phytoplankton competition analyzed by Károlyi et al. (2000) and Scheuring et al. (2000), and based on a simple kinetic model proposed by Toroczkai et al. (1998) and Károlyi et al. (1999). The work presented in these papers is especially relevant, as it has become a satisfying explanation for the so-called paradox of the plankton; see Sec. V.A.2 for more on this subject. The system consists of a cylinder that is surrounded by an open flow full of a background material $A$. The resource material $A$ is constantly introduced in the system, and two phytoplankton species B and C compete for it. Both species reproduce at time intervals $\tau$, but only if their centers are closer than $\sigma$ to material A. Furthermore, their reproductive rates $\gamma$ are proportional to $\sigma / \tau$, and they disappear with mortality rate $\delta$. The reproduction process and the competition are described by

$$
\mathrm{A}+\mathrm{B} \stackrel{\gamma_{\mathrm{B}}}{\rightarrow} 2 \mathrm{~B}, \quad \mathrm{~B} \stackrel{\delta_{\mathrm{B}}}{\rightarrow} \mathrm{A} \text { and } \mathrm{A}+\mathrm{C} \stackrel{\gamma_{\mathrm{C}}}{\rightarrow} 2 \mathrm{C}, \quad \mathrm{C} \stackrel{\delta_{\mathrm{C}}}{\rightarrow} \mathrm{A} .
$$

The computation starts when the whole free space around the cylinder is full of material A, and small droplets of species $B$ and $C$ are placed just in front of the cylinder. As A is the only existing resource for both species $B$ and $C$, traditional theory affirms that in a situation of total and homogeneous mixture the species with 
higher $\gamma / \delta$ eliminates the rest of competitors (Gause and Witt, 1935; Hardin, 1960). However, the result of the computation is totally different, as both species grow, spread around the cylinder following very thin filaments, and coexist for all times. As we have seen earlier, there is a chaotic set formed by infinite bounded orbits around the cylinder, and the species after a certain time tend to accumulate on the fractal unstable manifold of this chaotic set.

These results are in agreement with several experimental measurements, both in field observations and using remote sensing images. For example, Arístegui et al. (1997) studied the influence of eddies generated by the Canary Islands on chlorophyll distribution, making use of both hydrographic and biological observations from three different cruises. In this work, the experimental results are compared with computer simulations, and their remote sensing images show a fractal distribution of chlorophyll in the wake of the islands. Furthermore, field work developed also around the Canary Islands and presented by Barton et al. (1998) analyzed the existence of an upwelling filament, which is a consequence of the interaction of a topographically trapped cyclonic eddy with the outer edge of the coastal upwelling zone. They studied in detail the role of this filament and the cyclonic and anticyclonic eddies downstream of the islands in the transport and exchange of biogenic material, such as phytoplankton or fish larvae, and their influence on the increase of chlorophyll concentrations.

As can be imagined, it is in the field of ecological and environmental phenomena where we can obtain the most beautiful photographs of fractal structures in nature. For instance, Tél and Gruiz (2006) show a highquality collection of NASA photographs of natural chaotic advection phenomena. Wind vortices just after a high mountain in a Chilean island, plankton distribution around the Shetland Islands, and sea ice advected by the ocean around Kamchatka are only a few of the examples in which we can enjoy real cases of unstable manifolds associated with chaotic advection.

\section{WADA BASINS}

Although it is hard to imagine, it is possible to have three or more regions sharing the same boundary. Usually, three connected regions in two dimensions, for example, three countries, can coincide only at two points, but topologically this is not necessarily true for open sets. If we talk about basins, a basin B satisfies the Wada property if any initial condition which is on the boundary of one basin is also simultaneously on the boundary of two other (or more) basins. In other words, every open neighborhood of a point $x$ belonging to a Wada basin boundary has a nonempty intersection with at least three different basins. The first example of a system with this property was given by Yoneyama (1917), and another early reference to this phenomenon is due to Kuratowski (1924).

Therefore if a dynamical system satisfies the Wada property, the unpredictability is even stronger than if it only had fractal basin boundaries. If a trajectory starts close to any point in the boundary, it will not be possible to predict its future behavior, since its initial conditions could belong to any of the three basins.

The section is organized as follows. It starts with a topological approach to the Wada property, since it is not at all a trivial concept. Then, the key ideas of accessible periodic orbit and basin cell are defined. The section ends with a schematic presentation of the computational conditions that must be verified to assure that a basin is Wada.

\section{A. Topology of Wada basins}

From a topological point of view, a Wada basin boundary is an indecomposable continuum, a peculiar topological object that has been studied since the beginning of the 20th century. For an introductory approximation to the nature and history of indecomposable continua see Kennedy (1995). To define indecomposable continua precisely we first need the following definitions:

- A compact set $\mathrm{A}$ is any subset of $\mathbb{R}^{n}$ such that all its open covers have a finite subcover. This is the definition most commonly used, but the easiest to satisfy is to say that $A$ is a compact set if it is closed and bounded.

- A continuum A defined in $\mathbb{R}^{n}$ is a compact, connected subset of $\mathbb{R}^{n}$; see Sec. IV.A for the definition of connected set.

- If two sets $\mathrm{A}$ and $\mathrm{B}$ are continua, and $A \subseteq B$, then $\mathrm{A}$ is a subcontinuum of $B$. If $A \neq B$, then $A$ is a proper subcontinuum of $B$.

- A continuum A is decomposable if it can be written as the union of two overlapping proper subcontinua $\mathrm{B}$ and $\mathrm{C}$.

Most continua are decomposable. However, it is possible to find indecomposable continua, that is, continua that cannot be written as the union of two overlapping proper subcontinua.

In summary, such indecomposable sets are compact, metric, and connected sets with the strange property that when one attempts to divide them into two pieces, they split up into infinitely many pieces.

The first indecomposable continuum was discovered by Brouwer (1910), as a counterexample to the conjecture of Schoenflies that affirmed that the common boundary between two open, connected, and disjoint sets in the plane had to be decomposable (that is, the union of two proper, closed, and connected sets). It is remarkable that Brouwer affirmed that his construction could be modified so that the indecomposable continuum would become the boundary for any finite or countable number of disjoint, connected, simply connected sets in the plane. This can be recognized as the first reference to the Wada property. However, it is probably the remarkable curve of Birkhoff (1932) that is the 


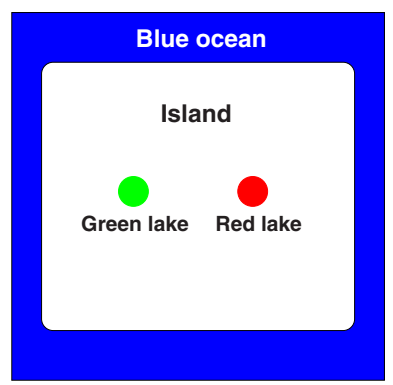

(a)

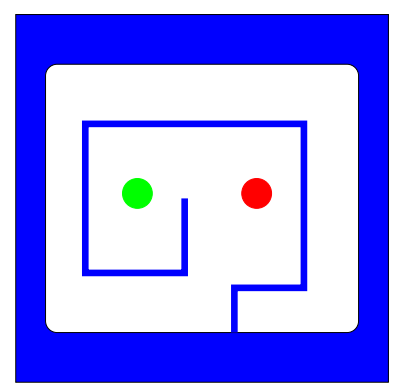

(b)

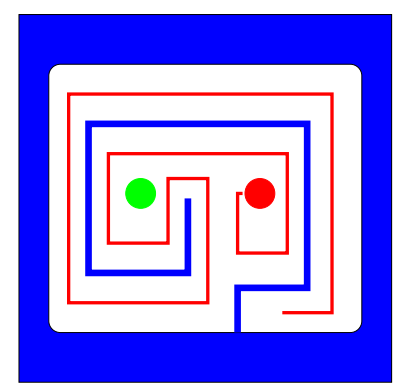

(c)

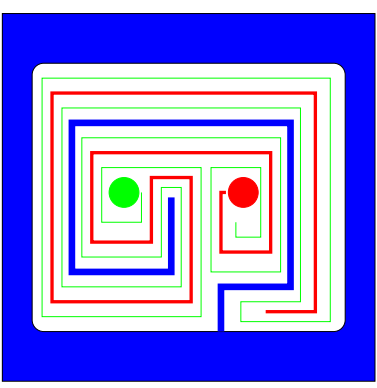

(d)

FIG. 10. (Color online) First steps to build the lakes of Wada. (a) The island has a green lake and a red lake. It is surrounded by the blue ocean. (b) Creation of the blue canal. (c) Creation of the red canal. (d) Creation of the green canal.

first example of an indecomposable continuum in a dynamical system. During the 1940s, Cartwright and Littlewood $(1945,1951)$ analyzed the forced van der Pol oscillator. They found that, under certain parameter values, a Poincaré map of this oscillator admits an invariant plane-separating continuum. They conjectured that this continuum contains an indecomposable continuum, but it was not until 1991 that Barge and Gillette (1991) proved rigorously that this continuum was an indecomposable continuum.

In order to cast some light on the work of Cartwright and Littlewood on the van der Pol oscillator, Smale (1967) presented the horseshoe maps that nowadays take his name. These peculiar structures were proved to contain an indecomposable continuum, and it is widely known that they are almost ubiquitous in nonlinear dynamics.

During the 1970s, Williams $(1967,1974,1979)$ continued the work on indecomposable continua, but instead of working with Cantor sets, he presented the concepts of branched manifolds and inverse limits, nowadays important mathematical tools to analyze nonlinear dynamics. Solenoids are fundamental examples of inverse limits on branched manifolds, and they are commonly indecomposable continua. Solenoids appear in differential equations and they are the limiting invariant set that occurs as a parameter is varied through a cascade of period doublings. In some sense, solenoids are to continuous systems what Cantor sets are to maps.

Finally, theorists have changed their view on indecomposable continua, and they no longer consider them "monstrous things created by set theoretic topologists for some evil (but purely mathematical) purpose" (Wallace, 1955). For example, strange attractors, fractal basin boundaries, and stable and unstable manifolds of chaotic saddles are known to be indecomposable continua (Sanjuán, Kennedy, Ott, et al., 1997). Furthermore, some authors affirm that they should be "expected" in a wide variety of dynamical systems, and they have appeared in many contexts [see Kennedy (1995), Sanjuán, Kennedy, Grebogi, et al. (1997), Sanjuán, Kennedy, Ott, et al. (1997) and Kennedy et al. (1999) for a detailed list of these applications].

The "lakes of Wada" are a useful example of how to construct three regions that satisfy the Wada property. The following explanation is due to Hocking and Young
(1961); see Fig. 10 for a schematic sketch of the first steps of the algorithm. In a blue ocean an island has a green lake and a red lake. From $t=0$ to $1 / 2$, a canal is dug from the ocean so that every piece of land on the island is within 1 mile of ocean water [see Fig. 10(b)]. From $t=1 / 2$ to $3 / 4$, another canal is dug from the red lake so that every piece of land on the island is within $1 / 2$ mile of red lake water [see Fig. 10(c)]. From $t=3 / 4$ to $7 / 8$, a third canal is dug from the green lake so that every piece of land on the island is within $1 / 4$ mile of green lake water [see Fig. 10(d)]. From $t=7 / 8$ to $15 / 16$, the first canal (the one filled with ocean water) is extended in such a way that every piece of land on the island is within $1 / 8$ mile of ocean water, and so on. After all the canals are dug, in the limit of $t=1$, the remaining land ( $L$ from now on) is the boundary of all canals. Every point of the island is a boundary point of both lakes and the ocean, and therefore is a Wada point.

We now analyze the nature of the lakes of Wada, which gives us an intuitive idea of what the Wada property means for a set. From a topological point of view, the remaining land $L$ after the construction of the canals is in fact a continuum because it is the intersection of a nested collection of continua. Furthermore, $L$ satisfies a property that is equivalent to being indecomposable: For every closed set $T$ that does not contain all of $L$, but contains a closed disk $S$ whose interior intersects $L$, $\mathrm{T} \cap \mathrm{L}$ consists of an uncountable collection of components. In the case of the lakes of Wada, for every small closed disk whose interior intersects $L$, the intersection between this small set and the remaining land consists of an uncountable number of arcs, plus several points in the boundary of the small closed disk.

The topology of the red lake, the green lake, and the oceanic region has not changed from the beginning of the construction $(t=0)$ to the end $(t=1)$. On the other hand, the land of the island has drastically changed its properties, as after the construction all closed sets whose interiors are intersected by the remaining land $L$ have infinite pieces of red, green, and ocean water, separated by infinitely thin pieces of land. No disk shows water of exactly two colors, no matter how small this disk is. If we zoom in on any region of the remaining land, we always have water of all three colors. If we zoom in on a point of water, we see only single-color water. In summary, the 


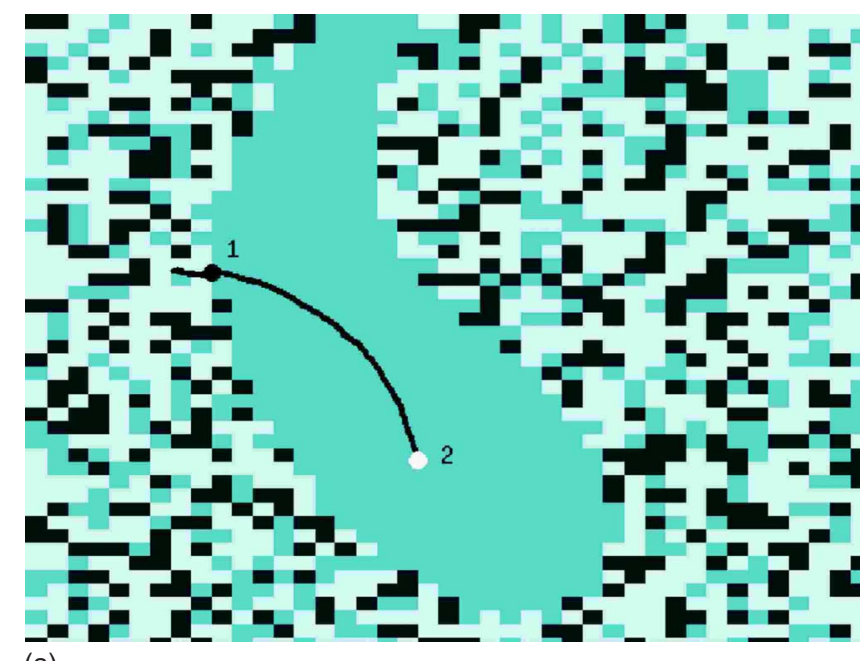

(a)

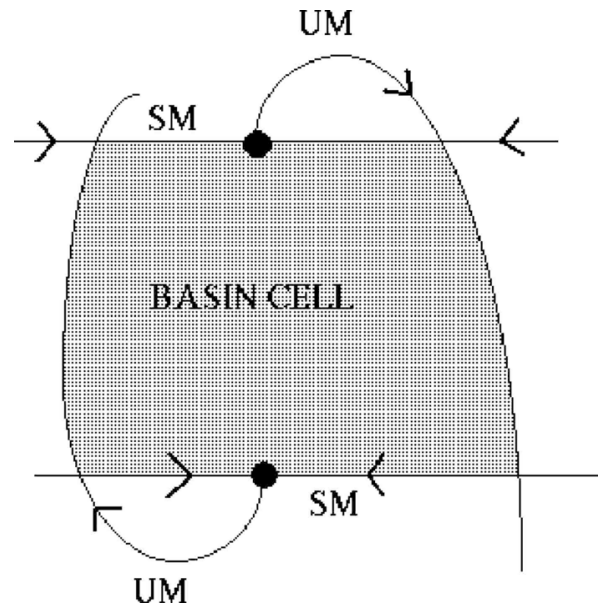

(b)

FIG. 11. (Color online) Accessible orbits and basin cells are key concepts for the verification of the Wada property: (a) Black point 1 is accessible from the blue basin, because it is the first boundary point that a curve hits, starting from the interior of the blue basin; (b) a basin cell is a trapping region formed by $n$ pieces of the stable and unstable manifolds of a boundary $n$-periodic orbit. From Aguirre and Sanjuán, 2002.

closure of any of the water sets coincides with the closure of the remaining land.

\section{B. Accessible periodic orbits and basin cells}

There are two concepts strongly related to the existence of Wada basins: the accessible orbits and basin cells. Both of them will be useful for the numerical verification of Wada basins.

\section{Accessible orbits}

A point $P$ in the boundary of a basin $B$ is accessible from that basin if a curve can be drawn, starting in the interior of the basin, in a way that this point $P$ is the first boundary point of the basin that the curve hits (Grebogi, Ott, et al., 1987; Alligood and Sauer, 1988; Hammel and Jones, 1989; Alligood and Yorke, 1992); see Fig. 11(a) for a schematic explanation of this concept. If the basin $B$ is fractal, there is typically a small subset of the boundary that is composed of accessible points, and due to the extreme complexity of the boundary they will not be accessible from any other basin.

\section{Basin cells}

Several definitions are necessary to understand what a basin cell is.

- A compact region is a simply connected, compact set with nonempty interior which consists of finitely many connected components.

- A trapping region is a compact region $T$ such that $\mathbf{F}(\mathrm{T}) \subset \mathrm{T}$ and $\mathbf{F}(\mathrm{T}) \neq \mathrm{T}$.

Therefore, if the particle enters the trapping region, it will never be able to escape from it. However, points that belong to the boundary of the trapping region $T$ need not enter $\mathrm{T}$ after iterations. The definition of the trapping region allows us to present an alternative and useful definition for the basin of attraction:

- A set B is the basin of attraction of $T$ if $B$ is the set of points that eventually map into the interior of $\mathrm{T}$, where $T$ is a trapping region.

This alternative definition avoids the problem of verifying that we have found all the attractors of a system.

Now we are prepared to define a basin cell:

- A basin cell is a trapping region constructed in such a way that its boundary is made out of $n$ pieces of stable and unstable manifolds of an $n$-periodic orbit that also lies on the boundary of the trapping region.

Basin cells were first introduced by Nusse and Yorke (1996). They deepened this concept and its influence on the geometry of mixing chaotic flows in Nusse and Yorke (2000). Figure 11(b) shows a clarifying diagram of a typical basin cell.

Basin cells provide much information about the basins they are associated with. First of all, they can be found only in dissipative systems. Furthermore, the corresponding basin can be seen as a central body formed by the basin cell plus several arms that connect to it. [The basin cell in Fig. 11(a) has only one associated arm, the wide channel that goes upwards.] There will be as many arms as the period of the periodic orbit that gives rise to the basin cell. The arms created in the basin cell are infinitely long and are stretched and folded in a very complicated manner, without crossing each other.

A diverging curve is a curve that starts inside a basin cell, chooses one of its arms, and follows it infinitely. Every diverging curve comes arbitrarily close to every point in the boundary of the basin. That is, the limit set of a diverging curve is the entire basin boundary. In fact, the fractal basin boundary of a basin that has a basin cell 
is invariant, and is equal to the closure of the stable manifold of the $n$-periodic saddle orbit that generates the basin cell. It is important to note that most basins do not have basin cells associated, although the existence of basin cells assures the fractality of the boundary. We end this summary of the properties of basin cells presenting the main result by Nusse and Yorke (2000), applicable for two-dimensional dissipative systems:

A basin B has a basin cell if and only if every diverging curve has the entire basin boundary of $\mathrm{B}$ as its limit set.

\section{Computational conditions to verify the Wada property}

It is easy to visualize from an intuitive point of view whether or not a dynamical system satisfies the Wada property. First, three or more basins must be defined. Then, we must zoom in on a region that contains part of the fractal basin boundary as many times as possible, and check that all three basins are present in the boundary no matter how small the region is. However, if our goal is to verify the Wada property with some rigor, the numerical verification presents several difficulties that must be solved, as the topology behind this property is not trivial. A thorough analysis of this subject was done by Kennedy and Yorke (1991) and Nusse and Yorke (1996), and some computational conditions were found to assure that a basin is Wada. While there are several equivalent secondary conditions that might be fulfilled, the main condition is the same for all cases.

Condition 1 (main condition). Let $\mathrm{P}$ be an unstable periodic orbit, accessible from a basin B. Its unstable manifold must intersect every basin.

The following argument from Kennedy and Yorke (1991) and Poon et al. (1996) explains qualitatively why the main condition is fundamental for verifying the Wada property. Figure 12 shows a schematic diagram of what happens when the unstable manifold of a periodic orbit $\mathrm{P}$ crosses three different basins. Suppose that $\mathrm{B}_{1}$, $B_{2}$, and $B_{3}$ are small disks that belong to basins 1,2 , and 3 . The unstable manifold of $P$ crosses all basins, and therefore the main condition is fulfilled. If we calculate the preimages of $B_{1}, B_{2}$, and $B_{3}$, that is, their images when the time $t$ goes backward, we find that they successively approach the stable manifold of $P$. The reason is that the distance between points of an unstable manifold contracts when time evolves backward. Furthermore, the preimages of $\mathrm{B}_{1}, \mathrm{~B}_{2}$, and $\mathrm{B}_{3}$ become exponentially stretched in the direction of the stable manifold, and get arbitrarily close to it. In the limit, all points on the stable manifold of the periodic orbit $P$ are boundary points, that is, there are points that belong to all three basins arbitrarily close to them. Therefore they are on the boundary of basins 1,2, and 3, and all points on the stable manifold of $P$ are Wada points.

The verification of condition 1 is a necessary but not a sufficient condition to verify that a system possesses the Wada property; one of the three alternatives for condition 2 must also be satisfied. Otherwise, there could be

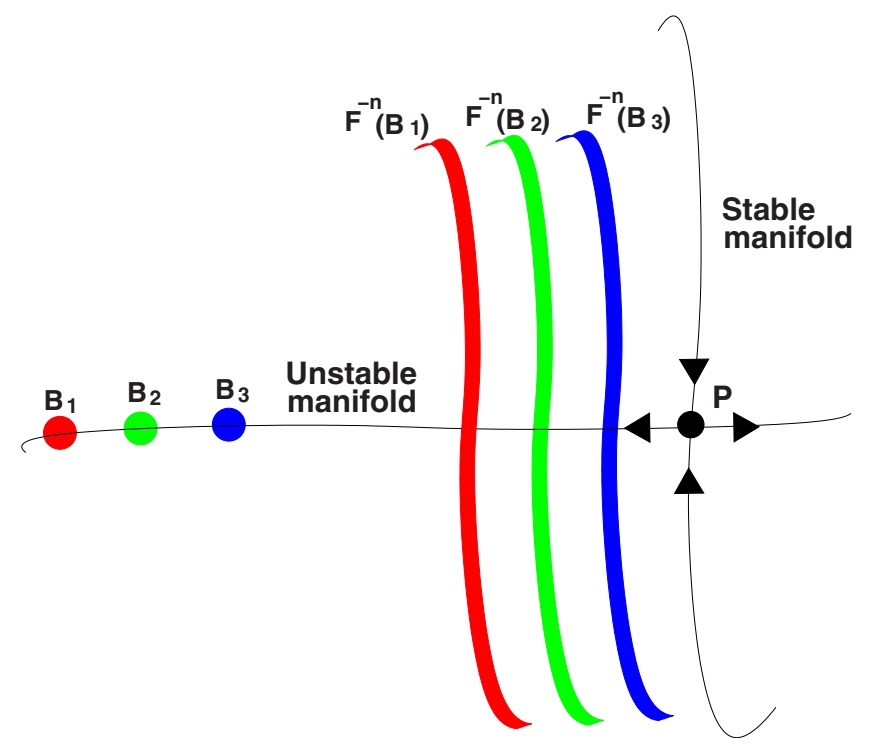

FIG. 12. (Color online) Three small sets belonging to three different basins and intersected by the unstable manifold of the accessible orbit $P$. Their preimages are exponentially stretched and successively approach the stable manifold of P.

unknown accessible orbits. Condition 1 only assures that the boundary created by the stable manifold of one accessible periodic orbit satisfies the Wada property. Other parts of the boundary could be non-Wada fractal or even smooth. For systems that show coexistence of Wada points and non-Wada points in the same boundary we propose the name of partially Wada basin boundaries.

Condition 2.A. If there is a periodic orbit $\mathrm{P}$ that satisfies condition 1, the basin B satisfies the Wada property if the stable manifold of such saddle point is dense in the boundary of all basins.

If we try to apply this secondary condition, we face two practical problems. First, it is hard to be sure that we have found all basins, and, second, it is even harder to prove rigorously that the stable manifold of the saddle point $P$ is indeed dense in the boundaries. For these reasons, Nusse and Yorke (1996) presented two theorems guaranteeing the existence of Wada basins, using easily verifiable numerical conditions. These two theorems provide conditions 2.B and 2.C.

Condition 2.B. If there is a periodic orbit $\mathrm{P}$ that satisfies condition 1 the basin $B$ satisfies the Wada property if such saddle point is the only accessible orbit from basin B. In case that there is more than one accessible periodic orbit; every unstable manifold must intersect all basins [Theorem 1 of Nusse and Yorke (1996)].

Condition 2.C. If there is a periodic orbit $\mathrm{P}$ that satisfies condition 1 the basin $B$ satisfies the Wada property if such saddle point generates a basin cell [Theorem 2 of Nusse and Yorke (1996)].

Computational steps to follow when verifying the Wada property. According to the theorems just presented, several numerical conditions must be satisfied. For many dissipative systems this should be an easy task, and in particular the software DYNAMICS (Nusse and Yorke, 
1997) has implemented the numerical methods that are needed for this purpose. In any case, the following steps are general enough to be applied to all those systems that are not analyzable by any particular software package, such as complicated dissipative systems or even Hamiltonian systems.

(1) Compute the attractors and graphically plot the corresponding basins. If we are working with an open Hamiltonian system, the role of the attractors and their basins is played by the exit modes and exit basins, respectively.

(2) Find as many accessible periodic orbits as possible. A powerful numerical method to do this is the accessible basin boundary straddle trajectory (ABST) method (Nusse and Yorke, 1991, 1997).

(3) Plot the unstable manifolds of every accessible periodic orbit and verify that they intersect all basins. The calculation of the unstable manifold of a periodic orbit can be implemented with the unstable manifold (UM) method (You et al., 1991; Nusse and Yorke, 1997).

(4) One of the following conditions must be checked:

(a) Verify the density of the stable manifold of the accessible orbit. This is always an open possibility, but in practice it is hard to do it rigorously. There are no implemented numerical methods to do this, and at the end the results are only approximate and must be checked with the naked eye.

(b) Verify that all accessible orbits have been found. In this case, we have the difficult goal of verifying that we have not missed any accessible periodic orbit. The randomly finding periodic orbit (RP) method (Nusse and Yorke, 1997) is an accurate method to find periodic orbits of a certain specified period (assuming the period is not too high). If the system is dissipative, the periods of all accessible periodic orbits must be the same, which obviously facilitates the task (Alligood and Yorke, 1992). While we never have the total certainty that there is not a slippery orbit that remains hidden, most systems only have one or very few accessible orbits, and in practice this is not a real problem.

(c) Construct a basin cell. Plot the stable and unstable manifolds of the accessible orbit, and construct a trapping region [using, for example, stable manifold (SM) and UM methods]. Remark that basin cells are only present in dissipative systems. The existence of a basin cell assures that there is only one accessible periodic orbit.

In summary, the main advantage of the concept of basin cell is that, in order to verify the existence of Wada basins in a system, condition 2.C is the only tool that does not need to make sure that all accessible orbits have been found. In this sense, the first step should be to try to find a basin cell associated with the basin that is being studied, and only if this method does not work should we try to make use of condition 2.B. When these methods are insufficient, we can try to verify that the stable manifold of the accessible orbit is dense in the boundary of all basins (condition 2.A), but, as already mentioned, it is extremely difficult to do this rigorously. (Unfortunately, in the context of Hamiltonian dynamics there is not a concept that is analogous to basin cells in dissipative systems. Therefore when we work with Hamiltonian systems, we are obliged to check either condition 2.A or 2.B.)

Finally, even if we make use of the condition related to basin cells, we are not proving rigorously that the basins are Wada. To do this, one should do the hard work of computing the accuracy of the calculations of the stable and unstable manifolds (Nusse and Yorke, 1996).

\section{WADA BASINS IN PHYSICAL SYSTEMS}

Although typical fractal basin boundaries have been shown to be very common in the context of nonlinear dynamics, the number of examples already studied of systems displaying the Wada property is still quite limited. However, they appear in numerical models and in experiments, as long as there are three or more different final states. Just as with fractal basins, Wada basins can be found in maps of any dimension, while threedimensional flows are needed to satisfy this peculiar property.

One interesting consequence of the Wada property is the existence of a high number of possible final states and the difficulty of predicting to which of them a certain initial condition leads. In fact, for many trajectories that start by the fractal boundary, a probabilistic approach is needed in order to predict the future behavior. Having Wada basin boundaries represents an intermediate situation between the softer case of having fractal basin boundaries and the stronger situation of riddled basins, where all deterministic prediction is lost.

This section has a similar structure to that of Sec. V, where examples of fractal basins were addressed. In fact, many typical fractal systems show Wada basins for certain values of the parameters. The first part is dedicated to dissipative systems, both maps and flows, and the second part is dedicated to conservative systems divided into chaotic scattering systems and open hydrodynamical flows. In particular, special attention is paid to the applicability of the computational conditions to verify the Wada property, and several cases are shown in detail for the sake of clarity.

\section{A. Dissipative systems}

\section{Maps}

a. One-dimensional maps

Very little work has been done on Wada basins related to one-dimensional maps. Vandermeer (2004) detected Wada basins in a circle map that models a predator-prey 

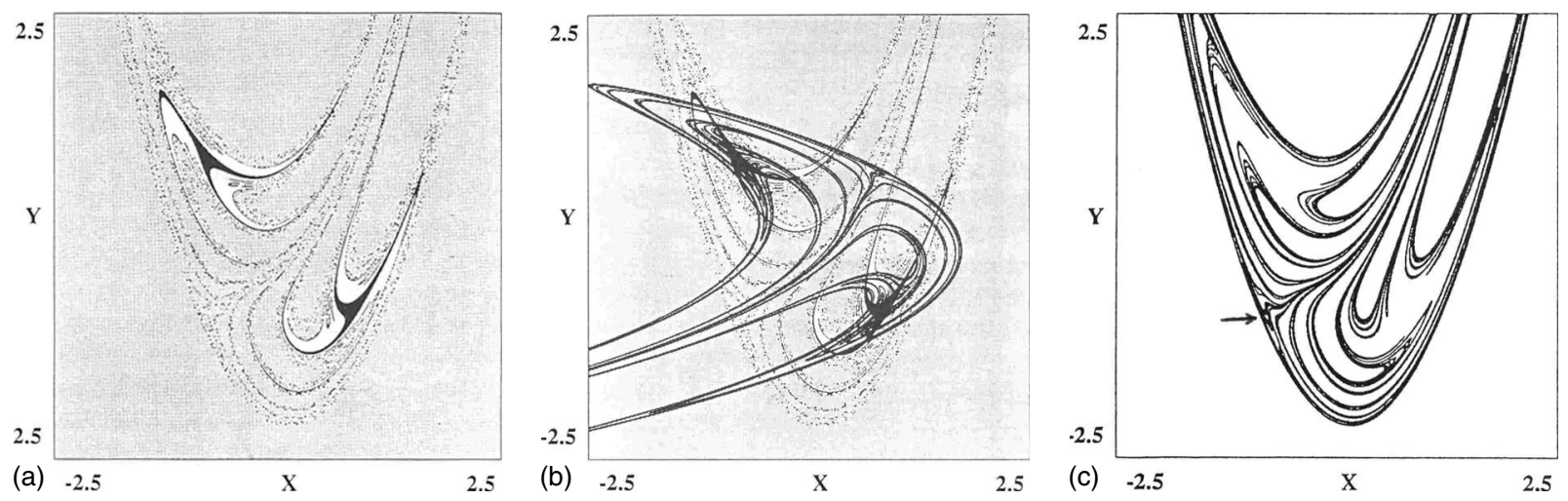

FIG. 13. Verification of the Wada property in the Hénon map with $a=0.7$ and $b=0.9$. (a) Fractal basin diagram. The gray basin is Wada, but not the black or white basins. (b) The unstable manifold of the only periodic orbit accessible from the gray basin intersects all basins. (c) The stable manifold of the only accessible orbit (marked with an arrow) coincides with the Wada basin boundary. From Nusse and Yorke, 1996.

ecological system, questioning the capability of these model systems to make precise predictions.

Breban and Nusse (2005) presented a sufficient and necessary condition that guarantees that three Wada basins emerge in one-dimensional maps. It is applicable for basins that appear in a tangent bifurcation for certain one-dimensional maps with negative Schwarzian derivative and at least two, but at most three, fixed point attractors. This condition, proved rigorously, is applied to check that several examples indeed satisfy the Wada property. For example, suppose that $\mathbf{T}(x)$ is the logistic map in the regime where the parameter $\lambda$ allows the existence of a period- $m$ attractor which has been created by a periodic point creating tangent bifurcation. The $\mathbf{T}^{m}(x)$ map defined by the logistic map $\mathbf{T}(x)$ applied $m$ times has $m$ basins (each of them associated with a period-1 attractor). McDonald et al. (1985) showed that these basins are fractal; see Sec. V.A.1 for more details. However, they also satisfy the Wada property. At the end of their work, Breban and Nusse apply the condition to more complex one-dimensional maps to show the generality of the analytic result.

\section{b. Two-dimensional maps}

The Hénon map [see Eqs. (16) and (17) for the mathematical expression] shows a varied typology of fractal basins, depending on the values of its two parameters $a$ and $b$. In some cases we can find situations in which the basins are only fractal. In some others all basins are Wada, while it is also possible to observe Wada basins and simple fractal basins coexisting (Nusse and Yorke, 1996). This wide variety of behaviors makes the Hénon map an especially interesting model to present as an example of the application of existing tools to verify the Wada property.

- Coexistence of Wada basins and non-Wada fractal basins. Following Nusse and Yorke (1996), we choose the parameters $a=0.7$ and $b=0.9$. According to the steps explained in Sec. VI.C, the basins of attraction are plotted. For these values of the parameters, there are three different basins of attraction, which are plotted in Fig. 13(a). Basin 1 is plotted in black and consists of the orbits that tend to a period- 2 attractor. Basin 2 is plotted in gray and is formed by the points whose orbits diverge to infinity, while basin 3 is plotted in white and its orbits tend to a period- 6 attractor. The second step is to find as many accessible periodic orbits as possible. If we start by studying the gray basin, two fixed points are found, but only one is accessible from that basin. To do this, they used the ABST method. Figure 13(b) shows how the unstable manifold of the only periodic orbit that is accessible from the gray basin indeed intersects all three basins (condition 1). They used the UM method to paint the whole unstable manifold, although we would only need a small piece of what is plotted to show that it intersects the three basins. Condition 2.B assures then that the gray basin is a Wada basin. Every point on the closure of the stable manifold is a Wada point. Figure 13(c) shows this set of Wada points. Looking at Fig. 13(a), it is clear that white and black basins share a smooth boundary in which no gray points are found. Therefore they do not satisfy the Wada property. Following the same steps this fact can be explained rigorously. Applying the ABST method one finds that there is only one accessible period- 6 orbit from the black basin, which is also the only accessible orbit from the white basin. However, its unstable manifold intersects only the black and white basins, but not the gray basin, and therefore condition 2.B tells us that these basins are not Wada.

- Wada basins in the Hénon map. Changing the parameter $a$ from 0.7 to 0.71 , the system still has three attractors, two of them periodic (period- 1 and period-6) and one at infinity. The basins are almost the same, but they show a fundamental difference: the smooth boundary that in the former case separated the black and white basins now is totally fractalized. In fact, now we find an accessible periodic orbit of period 1 from the gray basin, one of period 6 


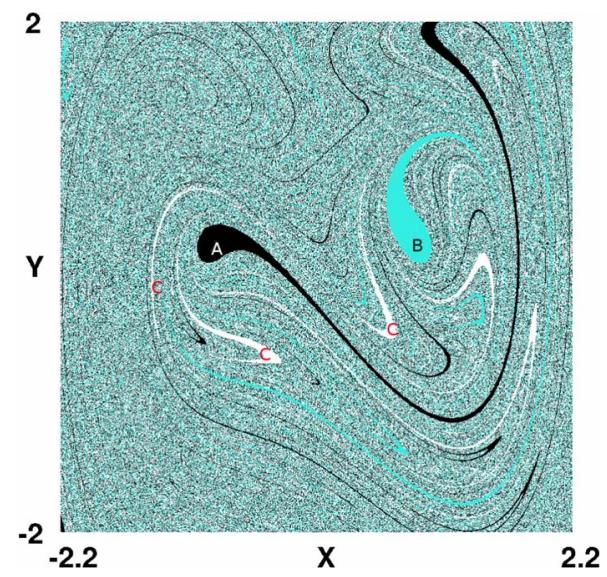

(a)

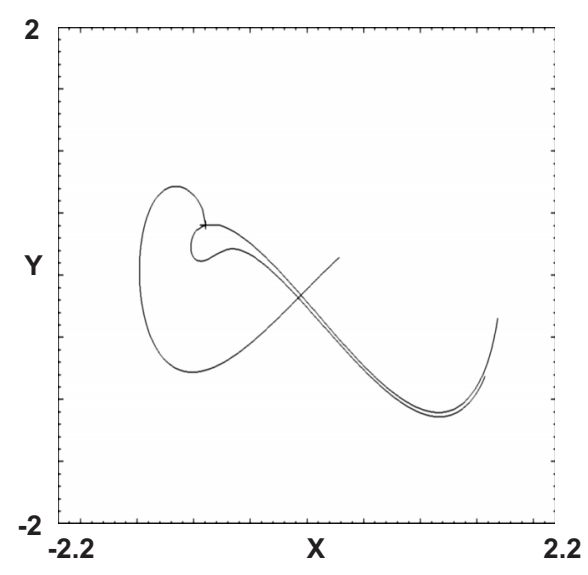

(b)

FIG. 14. (Color online) Verification of the Wada property in the Duffing oscillator. (a) Basins of attraction associated with two period-1 attractors (A and B) and one period-3 attractor (C). There are some regions where the likelihood of going to a certain attractor is bigger, but it is clearly observable that for most parts of the phase space the situation is very complicated. (b) Basin cell associated with a period- 1 attractor and its black basin. It is generated by the saddle marked with a cross. When the basin cell is compared with the figure of the basin diagram, it is observed that the branch of the unstable manifold intersects the three basins of attraction, which gives evidence that this basin is Wada. From Aguirre and Sanjuán, 2002.

from the black basin, and one of period 18 from the white basin, and their unstable manifolds indeed intersect all three basins. Therefore, following conditions 1 and 2.B, we know that gray, black, and white basins shear the same boundary and satisfy the Wada property.

\section{Flows}

Most oscillators defined in a phase space of dimension 3 or more, and in the regimes where there are three or more basins, have turned out to show Wada basins for certain values of their parameters. The first example in the literature is the forced damped pendulum (Kennedy and Yorke, 1991). In this work it was shown numerically that the unstable manifold of an accessible saddle point intersects three basins (condition 1). Furthermore, a fairly technical proof was given for the density of the stable manifold in the boundary of the three regions (condition 2.A). The difficulty of this proof is a clear evidence of the unsuitability of applying condition 2.A to verify the Wada property. On the other hand, Nusse and Yorke (1996), studied the same case, but this time making use of the new concept of basin cell. In fact, the fractal basins associated with nonlinear oscillators typically show basin cells, and therefore it is possible to apply condition 2.C [Theorem 2 of Nusse and Yorke (1996)], which is by far the easiest and most robust way to check that a dissipative system satisfies the Wada property.

Recently, the Duffing oscillator has also been shown to satisfy the Wada property (Aguirre and Sanjuán, 2002; Aguirre et al., 2003). Figure 14(a) shows its numerically computed basin diagram, where $\delta=0.15, \alpha=\beta=\omega$ $=1$, and $\gamma=0.245$; see Eq. (20) for the mathematical expression of the Duffing oscillator. For these values of the parameters, there are three attractors (two fixed points and a period- 3 orbit), and the corresponding basins of attraction have been colored black, blue, and white. The fractality calculated as the uncertainty dimension is $D$ $=1.98 \pm 0.01$ (where $D=1$ means nonfractality and $D=2$ means total fractality), which indicates a highly fractal structure. It is verified that there are three basin cells, and the basin cell associated with the period-3 attractor is formed by three disconnected basin cells. A picture with the basin cell for the black basin is shown in Fig. 14(b). This basin cell is formed by two stable manifolds and one unstable manifold of the accessible unstable periodic orbit marked with a cross in the plot. Comparison of this picture with Fig. 14(a) shows that the unstable manifold crosses all three basins. They obtain the same results for the other two basins, giving evidence that, for these values of the parameters, the Duffing oscillator satisfies the Wada property.

The existence of fractal and Wada basins is starting to be taken into account in the development of mechanical devices. For instance, Bellido and Ramírez-Malo (2006) studied the mechanical problem concerning a sliding rigid body, periodically forced and subjected to dry friction, which is a simple model for an articulated sliding vehicle undergoing lateral oscillations. This system shows a rich variety of nonlinear phenomena such as periodic and chaotic states, different bifurcations, and fractal and Wada basins, and all this should be considered when building a real device.

\section{B. Conservative examples}

The rigorous verification of the Wada property is in general much harder when working with conservative systems than when dealing with dissipative systems. The main reason is that we cannot make use of the existence of basin cells, but it is also important that the available 

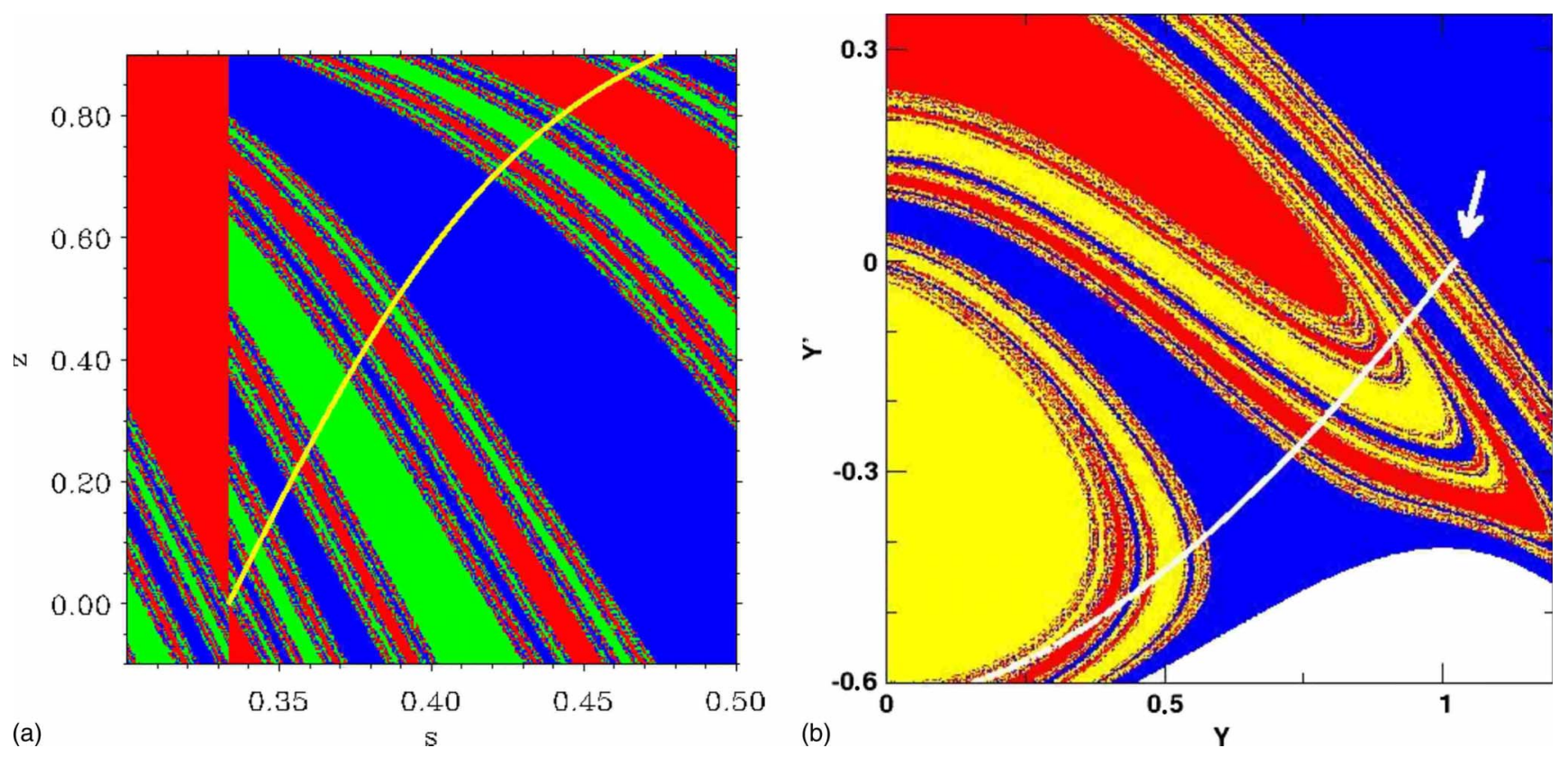

FIG. 15. (Color online) Verification of the Wada property in open Hamiltonian systems: (a) Wada basins associated with the three-hard-disk triangular configuration. From Poon et al., 1996. (b) Wada basins associated with the Hénon-Heiles system. From Aguirre et al., 2001. In both cases the unstable manifold of the only accessible periodic orbit [placed in (1/3,0) in (a) and marked with an arrow in (b)] intersects all three basins.

software and the numerical methods therein were mainly developed for dissipative systems. In most cases found in the literature of Wada basins in conservative systems, the main condition, which affirms that the unstable manifold of an accessible periodic orbit must intersect three basins, is always numerically fulfilled without any problem, but the situation is completely different with the secondary conditions. As condition 2.C is reserved for dissipative systems, one must either find all accessible periodic orbits or show that the stable manifold of the accessible orbit is dense in the boundary of all basins. In practice, neither of the two targets can be achieved rigorously, so different methods have been tried, some numerical and some qualitative, to obtain at least a sufficient certainty. In many cases, actually, only the main condition is satisfied, ignoring the secondary conditions. It is important to note that in these cases it may happen that there are accessible orbits that have not been found, and therefore one is only assuring that part of the boundary (the boundary that coincides with the stable manifold of the known accessible orbit) has Wada points. Other parts of the boundary, however, could be only fractal or even smooth.

\section{Chaotic scattering}

As already mentioned, Bleher et al. (1988) showed that it is typical of chaotic scattering systems to present fractal basin boundaries separating the initial conditions that make the particle escape from the system through different exits. Poon et al. (1996) went further in this line of research and showed in a simple two-dimensional billiard that these basins are not only fractal, but also satisfy the property of Wada (as long as there are three or more exits, obviously). This was the first time the concept of Wada basins was related to a conservative system. Furthermore, Poon et al. claimed that Wada basins would be generally found in open systems.

The model used by Poon et al. is a two-dimensional billiard consisting of three hard disks of radius $R$, whose centers are on the vertices of a triangle of side $L>2 R$. This configuration defines its scattering region as the bounded area between the disks and the triangle formed by their centers, and it has three exits of size $w=L-2 R$. A typical test particle moves at constant speed in the scattering region, suffering elastic collisions with all three disks, until it crosses one of the three exits of size $w$ and escapes to infinity. This system was first studied by Eckhardt (1987), extensively analyzed in the classical, semiclassical, and quantum regimes by Gaspard and Rice (1989a, 1989b, 1989c) and examined in the context of microscopic deterministic diffusion by Nobbe (1995), and Klages and Dellago (2000). A review of the properties of its dynamics can be found in Cvitanovic et al. (2005). The three hard disk configuration is one of the simplest and most general open Hamiltonian systems, and is a paradigm for low-dimensional chaotic scattering. For these reasons and for the sake of universality we present it here with some detail.

Figure 15(a) shows a part of the exit basin diagram for the system. The color code to plot this exit basin diagram is red, green, and blue, depending on the exit the particle chooses to escape from the system. The variables are $(s, z)$, where $s$ represents the arclength of each collision with the hard disks normalized from 0 to 1 , and $z$ satisfies $z=\cos \theta$, where $\theta$ is the angle the trajectory makes with respect to the forward tangent after each 
collision [see Poon et al. (1996) for more details]. It is clear that variables $s$ and $z$ are defined only while the particle is inside the scattering region. It has been shown that this mapping is area preserving (Birkhoff, 1927; Berry, 1981). Furthermore, a segment of the unstable manifold of one accessible periodic orbit is plotted, and it clearly intersects all basins. For open systems, the most suitable accessible periodic orbits are extremely unstable periodic orbits, which act as frontiers of the scattering region. They are known as Lyapunov orbits (Contopoulos, 1990), and when any orbit crosses one of them in the outer direction, that is, its velocity components pointing outward, the particle is forced to escape to infinity and never returns. Every system shows as many Lyapunov orbits as exits. Furthermore, the Lyapunov orbits are accessible by construction.

In addition to verifying that the stable manifold of the Lyapunov orbit is dense in the boundary, Poon et al. used a qualitative explanation of the existence of Wada basins, based on the one presented when discussing condition 1 in Sec. VI.C. The basic idea can be explained as follows. We choose a boundary point $\beta$ and a small disk $D_{\epsilon}(\beta)$ of radius $\epsilon$, centered at $\beta$. The goal is to show that there are points of all three basins inside $D_{\epsilon}(\beta)$, no matter how small $\epsilon$ is. Now we consider a horizontal line segment $L_{\epsilon}(\beta)$ through $\beta$ contained in $D_{\epsilon}(\beta)$. As we are working with an open system, there is a chaotic saddle formed by the set of Lebesgue measure zero of orbits that will never escape from the scattering region for both $t \rightarrow \infty$ and $t \rightarrow-\infty$. Furthermore, the fractal basin boundary coincides with the stable manifold of the chaotic set, and consequently is constituted by the orbits that do not escape from the scattering region, no matter how long we wait. From Fig. 15(a) we see that the slope $d z / d s$ of the stable manifold of the chaotic set is negative, while the slope of the unstable manifold is positive, as both manifolds are symmetric. Taking all this into account, $L_{\epsilon}(\beta)$ is transverse to the stable manifold, and when we iterate the orbit $\beta$ it will approach the chaotic set. As the system is hyperbolic (Bunimovich and Sinai, 1980) (that is, there are no tangent crossings between the stable and the unstable manifolds of the invariant set), the image of $L_{\epsilon}(\beta)$ will expand in the direction of the unstable manifold, until it is so long that crosses all three basins, assuring that inside $D_{\epsilon}(\beta)$ there are points of all basins. Therefore the point $\beta$ is a Wada point. As $\beta$ might be any point of the stable manifold, that is, the fractal boundary, the whole boundary is Wada.

The work of Poon et al. (1996) was done on an open billiard, one of the two types of open Hamiltonian system. Aguirre et al. (2001) extended the verification of the existence of Wada basins to the other type, that is, open two-dimensional potentials. Using as a model the Hénon-Heiles system (see Secs. IV.D.2 and V.B.1 for more information on this system), they verified that the unstable manifold of the Lyapunov orbit associated with each of the three basins indeed intersects all three basins, as shown in Fig. 15(b). Following condition 2.B, they verified that each Lyapunov orbit is the only un- stable periodic orbit that is also accessible from its corresponding basin. For this purpose, they used an argument based on the work of Alligood and Yorke (1992), where it is proved that, if all the periodic points in the boundary of a basin are hyperbolic, and there exists an accessible periodic point of minimum period $q$, then every accessible point in the basin boundary either is a periodic point of minimum period $q$ or is in the stable manifold of such a periodic point. From here, one can assume that in the basin boundaries of the HénonHeiles, only period-1 orbits can be found. This theorem provides us with a powerful tool to verify condition 2.B, as it is easy to find period- 1 orbits in a basin. After searching for period-1 orbits, a few periodic orbits were found, although none of them were in the boundary. Therefore enough numerical evidence is obtained to affirm that the three basins of the Hénon-Heiles system satisfy the Wada property. This strategy is applicable to any other two-dimensional potential with escapes.

Furthermore, it is worth referring to the possible appearance of Wada basins in closed systems that are artificially opened with three or more exits. As we know, in most of these cases fractal basins coexist with smooth basins, in what are usually known as intertwined basins. For these situations, it is usually possible to verify the main condition and check that the unstable manifold of an accessible periodic orbit intersects all basins, as done by Sanjuán et al. (2003) for a Hamiltonian map or by da Silva et al. (2002) and Portela et al. (2007) for maps related to plasma dynamics in tokamaks. However, if the basins are intertwined, there are necessarily parts of the boundary that are not fractal (and obviously not Wada either), and the secondary conditions must fail. These are examples of what we have named partially Wada basins; see Sec. VI.C for more details.

The notion of dissipative chaotic scattering caused by introducing a weak dissipation in a map was introduced by Motter and Lai (2001), and its consequences for the fractal dimension were analyzed by Seoane et al. (2007). This idea was applied to the Hénon-Heiles system by Seoane et al. (2006), showing that Wada basins survive the addition of weak dissipation in a typical open Hamiltonian system. Finally, note also that a study of chaotic scattering problems in a noisy environment was carried out by Seoane and Sanjuán (2008), where they showed that Wada basins are destroyed when a small amount of noise is introduced into the system.

\section{Open hydrodynamic flows}

While studying fractal basin boundaries associated with chaotic advection, Toroczkai et al. (1997) soon realized that, if Poon et al. (1996) had shown that Wada basins are a ubiquitous phenomenon in chaotic scattering systems, they should also appear naturally in the environment of open hydrodynamic flows. The problem of the existence of more than one basin when there is only one upstream and one downstream direction had already been solved by coloring the upstream region (the so-called inflow experiment) or the downstream region 

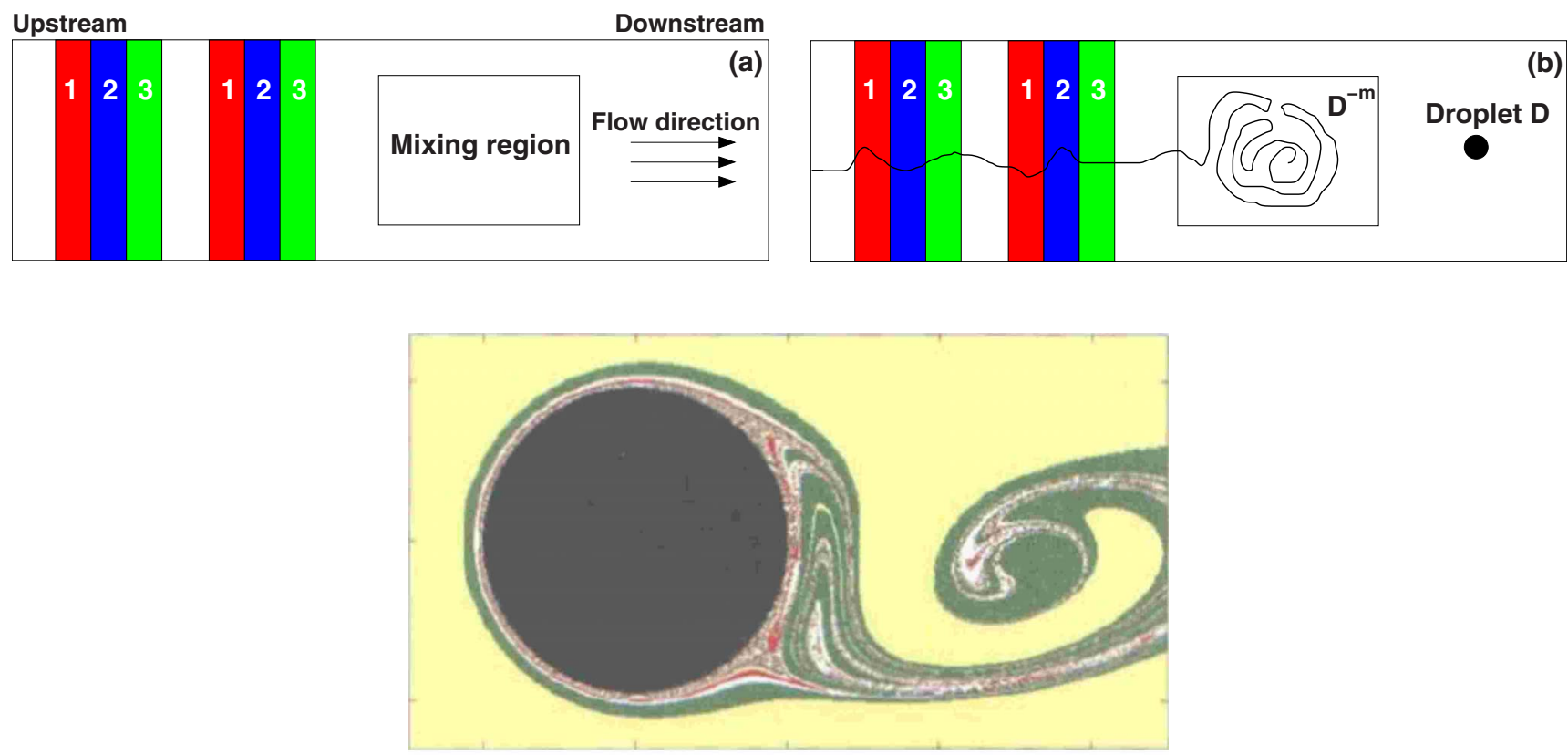

(c)

FIG. 16. (Color online) Verification of the Wada property in open hydrodynamic flows. (a) Sketch of an open flow around an obstacle, where the upstream region has been dyed with $n \geqslant 3$ vertical stripes. The mixing region is also plotted. The fluid satisfies the Wada property if the stable manifold of the chaotic saddle crosses all vertical bands. (b) A small droplet $D$ is placed in the downstream region, just on the unstable manifold. The preimage $D^{-m}$ of the droplet as $m$ grows indefinitely, tends to the chaotic set inside the mixing region, and shows a branch toward $\infty$ in the inflow region following the stable manifold. (c) Wada basins due to four different colors in a von Karman vortex street problem around a cylinder. (c) from Toroczkai et al., 1997.

(the so-called outflow experiment) with several different dyes; see Sec. V.B.2 for more details.

Taking all this into account, and making use of the inflow experiment point of view, it is easy to show that for a time-dependent open hydrodynamic flow that shows a chaotic saddle, where $n \geqslant 3$ vertical bands in the far upstream direction have been colored with $n$ different dyes as sketched in Fig. 16(a), the fluid shows Wada basins if the stable manifold of the chaotic saddle crosses all vertical bands (Toroczkai et al., 1997). Take a droplet $D_{\epsilon}(\beta)$ of radius $\epsilon$, centered at $\beta$, where $\beta$ is a point of the unstable manifold of the chaotic set associated with the system [Fig. 16(b) shows a basic diagram of this situation]. If one calculates its preimages $D_{\epsilon}^{-m}(\beta)$ when $m$ $\rightarrow \infty$, one finds that the circular set will get longer and longer, showing a very complicated structure inside the mixing region, as well as a branch towards $\infty$ in the inflow direction. This is due to two facts. First, the center of the droplet will tend to the chaotic set, because $\beta$ was taken exactly on the unstable manifold. Second, the preimages of the points of the droplet that were close to but not exactly on the unstable manifold, enter the mixing region, and finally escape toward infinity in the inflow direction following the stable manifold. For this reason, there is a finite $m$ such that the $m$ preimage of the original droplet will be so long that it will cross all the original transverse bands, assuring that it is formed of points of all colors. Obviously, if $D_{\epsilon}^{-m}(\beta)$ consists of points that belong to all colors, its image after $m$ iterations, that is, the original droplet $D_{\epsilon}(\beta)$, will also be formed of points of all colors. As the only prerequisite for $D_{\epsilon}$ is that it is centered on the unstable manifold, but its radius $\epsilon$ can be as small as desired, it is proved that all points on the unstable manifold (that is, on the basin boundary) are Wada points and therefore the system indeed satisfies the Wada property. As an example, Fig. 16(c) plots the Wada mixture of four different basins in a von Karman vortex street problem around a cylinder.

There are certain differences between this result and the numerical conditions presented to verify the existence of Wada basins in Sec. VI.C. First, the fractal boundary between the colored sets coincides with the unstable manifold of the chaotic saddle instead of coinciding with the stable manifold. Second, the Wada condition is that the stable (and not the unstable) manifold crosses all original basins. The reason is that we have defined income regions (the inflow experiment) instead of exits in the outcome regions, as is usual in chaotic scattering problems (the outflow experiment). As explained in Sec. V.B.2, the point of view of the inflow experiment is especially useful because it provides us with a nice tool to visualize Wada basins experimentally, just by dying the upstream region with several different colors. Obviously, if the vertical bands are chosen in the far downstream direction, as if they were the different exits that the tracers can choose to escape from the mixing region, we recover the typical results: the fractal basin boundary will coincide with the stable manifold of the chaotic set, and will be Wada if the unstable mani- 
fold crosses all the vertical bands located in the far downstream region.

\section{RIDDLED BASINS}

In the previous sections we have considered chiefly fractal basins, for which the problems with final state sensitivity are more severe than for smooth basins, in the sense that our ability to predict the asymptotic dynamical state is diminished for fractal basins, when compared to smooth ones. However, it turns out that an even more challenging situation takes place for the socalled riddled basins, since they virtually defy any attempt to improve the final state predictability. In other words, while for fractal basins a large reduction of initial state uncertainty is necessary to yield a better predictability, for riddled basins no improvement at all would be achieved by means of this procedure. This occurs because of the conspicuous mathematical structure of riddled basins, which is the subject of this section. We begin by reviewing the conceptual and historical background of this matter, with an outline of the basic topological and metric definitions needed to characterize mathematically riddled basins. We first analyze, as a simple example, a low-dimensional dynamical system for which the occurrence of riddled basins can be more easily shown. Next we consider a number of physical examples for which riddled basins have been theoretically and even experimentally described, in particular with respect to the basin structure of coupled dynamical systems. To conclude, we also review some alternative concepts related to riddled basins, but with different conceptual and mathematical frameworks (riddledlike basins).

\section{A. Introduction and historical aspects}

Chaotic systems having certain symmetries and quite general properties may present basins of attraction extremely interwoven in a more severe sense than we have dealt with in the preceding sections. This phenomenon has been called riddling, and the effect it causes in the dynamical system is the production of riddled basins. A dynamical system may have a chaotic attractor $A$ whose basin of attraction is riddled with "holes" (in a measuretheoretical sense) belonging to the basin of another (not necessarily chaotic) attractor $B$. In brief, riddling means that every point in the basin of attraction of attractor $A$ has pieces of the basin of attraction of attractor B arbitrarily nearby. These latter pieces represent the measure-theoretical holes we are referring to.

The physical consequences of riddling may be quite serious in terms of our ability of predicting what attractor the trajectory originating from a given initial condition asymptotes to. Let $\mathrm{P}$ be an arbitrary point belonging to the basin of the chaotic attractor $A$. If the basin of $A$ is riddled by the basin of the other attractor $B$, then a small ball of radius $\epsilon$ centered at $\mathrm{P}$ has a nonzero fraction of its volume belonging to the basin of $\mathrm{B}$, irrespec- tive of how small the radius $\epsilon$ might be. Hence if we regard this $\epsilon$ ball as an uncertainty neighborhood related to the (numerical or experimental) determination of the initial condition, the resulting trajectory always has a positive probability of falling into the basin of the other attractor. In other words, the probability of escaping from the basin of attractor $A$ is nonzero for every uncertainty $\epsilon$. Consequently, predicting what will be the final state of the system becomes more difficult than in the previously described cases of fractal basins.

Basin riddling was named and described by Alexander et al. (1992), who also presented a variety of illustrative examples chosen from discrete maps. Independently, riddling was also noticed by Pikovsky and Grassberger (1991) in a study of coupled maps. Several authors (Sommerer and Ott, 1993b; Ott et al., 1993, 1994; Hayli and Vidovic, 1994) presented a physical example for which riddling may occur, namely, a particle moving under the influence of three forces: friction, an external periodic force, and a force due to a scalar potential. Moreover, locally riddled basins were found to occur in coupled dynamical systems exhibiting synchronization of chaos (Fujisaka and Yamada, 1983; Pecora and Carroll, 1990), leading to the so-called attractor bubbling and on-off intermittency (Ott and Sommerer, 1994). In the latter context, the occurrence of riddling for coupled nonlinear electronic circuits was experimentally demonstrated (Ashwin et al., 1994; Heagy et al., 1994b; Parmenter and $\mathrm{Yu}, 1994)$. Moreover, riddling may occur also in the parameter space (Lai and Winslow, 1994).

The mathematical properties of the transition from fractal to riddled basins were explored (Ashwin et al., 1996, 2002; Ashwin and Breakspear, 2001). The transition to riddling has been proved to occur due to a variety of bifurcations (Lai et al., 1996; Kapitaniak et al., 1998; Yang and Pikovsky, 1999), mainly related to the transition to synchronization of chaos (Kapitaniak and Maistrenko, 1998, 1999; Maistrenko et al., 1998, 1999a; Manscher et al., 1998); while the influence of noise in riddled basins was investigated (Lai and Grebogi, 1996; Lai, 2000). The basins are called intermingled when each basin is riddled with holes belonging to other basins (Alexander et al., 1992; Kan, 1994; Hofbauer et al., 2004; Pereira et al., 2008).

There is a large number of examples of riddling in dynamical systems of physical and biological interest. Riddled basins have been described in mechanical systems, like coupled elastic arches (Woltering and Markus, 1999), and were generally found to occur in spatially extended dynamical systems, like coupled map lattices (Ashwin, 2005). Other applications include ecological population models (Cazelles, 2001a, 2001b; Hofbauer et al., 2004), learning dynamical systems (Nakajima and Ueda, 1996), chemical reactions of the BelouzovZhabotinsky type (Woltering and Markus, 2000a), and models of interdependent open economies (Yousefi et al., 2000).

Since the mathematical requirements for riddling are rather restrictive, ensuing works dealt with situations in which the formal definition is not strictly applicable, but 
with physical consequences similar to riddling. These situations can be collectively called riddledlike basins. In this category we can include the influence of symmetry breaking, or catastrophe of riddling (Lai, 2000; Viana and Grebogi, 2001); pseudoriddling, or the riddling in periodic windows (Lai and Grebogi, 1999, 2000; Terry and Ashwin, 2000; Lai, 2001), riddledlike basins of transient regime (Woltering and Markus, 2000b), practical riddling (Blazejczyk-Okolewska et al., 2000; Kapitaniak, 2001), and uncertain basins created by open Hamiltonian systems when the exits shrink (Aguirre and Sanjuán, 2003); see Sec. IX.B.2 for more information on the nature and the experimental verification of uncertain basins.

\section{B. Basic definitions}

For many applications it suffices to define an attractor as a volume-zero subset of the phase space to which all trajectories originating in a given region (called its basin of attraction) asymptote (see Sec. II.A). However, the possibility of exotic sets like riddled basins to occur in physically relevant dynamical systems requires a more comprehensive definition of attractor, as that proposed by Milnor (1985): let $\mathrm{H}$ be the phase space in which the dynamical system $\mathbf{F}$ is defined. At first we may think of $\mathbf{F}$ as a discrete-time map, but continuous-time flows can be also described by $\mathbf{F}$, if Poincaré sections are taken. A closed subset $A \in H$ is said to be an attractor of $\mathbf{F}$ if it satisfies the following conditions:

- A has a basin of attraction, denoted $\beta(\mathrm{A})$, of positive Lebesgue measure (volume) in the phase space $\mathrm{H}$.

- $\mathrm{A}$ is a compact set with a dense orbit. In the Milnor definition of attractor, the basin of attraction does not need to include the whole neighborhood of the attractor.

If the basin of attraction of $A$ has positive Lebesgue measure, we call A a weak Milnor attractor (Hasler and Maistrenko, 1997).

The basin of a chaotic attractor $A$ is riddled if its complement intersects every disk of the phase space $\mathrm{H}$ in a set of positive Lebesgue measure (Alexander et al., 1992). Roughly speaking, the term "disk" has the meaning of phase-space volumes of all sizes. When the basin of attraction of $A$ is riddled with holes belonging to the basin of another attractor B, we can say that, if a randomly chosen point has a positive probability of being in $\beta(\mathrm{A})$, then it also has positive probability of not being in $\beta(\mathrm{A})$. In the latter case, the point belongs to the other basin of attraction $\beta(\mathrm{B})$.

This measure-theoretical definition implies the following set of conditions under which riddled basins occur in a dynamical system (Ott et al., 1993):

(1) There is an invariant subspace $\mathrm{M} \in \mathrm{H}$ whose dimension $d_{M}$ is less than that of the phase space $d_{H}$.

(2) The dynamics on the invariant subspace $M$ has a chaotic attractor $A$.
(3) There is another attractor B, chaotic or not, and not belonging to the invariant subspace $M$.

(4) The attractor $A$ is transversely stable in the phase space $H$, i.e., for typical orbits on the attractor the Lyapunov exponents for infinitesimal perturbations along the directions transverse to the invariant subspace $M$ are all negative.

(5) A set of unstable periodic orbits embedded in the chaotic attractor $A$ is transversely unstable. As a consequence, at least one of the Lyapunov exponents along directions transverse to $M$, although negative for almost any orbit of $A$, experiences positive finite-time fluctuations.

Condition 1 is a consequence of the system having some symmetry which enables it to display an invariant subspace $\mathrm{M}$, in the sense that, once an initial condition is exactly placed on $\mathrm{M}$, the resulting trajectory cannot escape from $\mathrm{M}$ for further times. To have riddling, it is necessary to have a dense set of points with zero Lebesgue measure in the attractor lying in the invariant subspace which are transversely unstable, thus it is necessary that this attractor be chaotic (condition 2). The existence of another attractor (condition 3) is necessary for the basin of an attractor to be riddled with holes belonging to the basin of this second attractor. In the special case of intermingled basins, there must be at least two attractors lying in different invariant subspaces, and the basin of each attractor is pierced with holes containing initial conditions belonging to the basin of the other attractor (Alexander et al., 1992; Kan, 1994; Hofbauer et al., 2004; Pereira et al., 2008).

If the transverse Lyapunov exponents of typical orbits lying in the invariant subspace $M$ are all negative (condition 4), then $A$ is an attractor in the weak Milnor sense, and its basin has positive Lebesgue measure. Condition 5 states that, while the invariant subspace $M$ is still transversely stable, there will be trajectories on the attractor A that are transversely unstable. Condition 4 can be quantitatively checked by computing the maximal Lyapunov exponent along a transversal direction to $\mathrm{M}$. Verifying condition 5, on the other hand, would require the determination of transversely unstable periodic orbits embedded in the attractor $A$. This is feasible only for a few dynamical systems (Lai et al., 1996; Viana and Grebogi, 2001; Pereira et al., 2007). In most situations we resort to other ways to verify the existence of finite-time fluctuations by computing the finite-time Lyapunov exponents.

In order to discuss conditions 4 and 5 in a quantitative setting, it is useful to define the finite-time Lyapunov exponents for a $d_{H}$-dimensional map $\mathbf{F}$. Let $n$ be a positive integer and $\mathbf{D F}^{n}\left(\mathbf{x}_{0}\right)$ be the Jacobian matrix of the $n$-times iterated map, with entries evaluated at an initial condition $\mathbf{x}_{0} \in A$. Suppose that the singular values of $\mathbf{D F}^{n}\left(\mathbf{x}_{0}\right) \quad$ are $\quad$ ordered: $\quad \xi_{1}\left(\mathbf{x}_{0}, n\right) \geqslant \xi_{2}\left(\mathbf{x}_{0}, n\right) \geqslant \cdots$ $\geqslant \xi_{d_{H}}\left(\mathbf{x}_{0}, n\right)$. Then, the $k$ th time- $n$ Lyapunov exponent for the point $\mathbf{x}_{0}$ is defined as (Viana and Grebogi, 2001) 


$$
{\tilde{\lambda_{k}}}_{k}\left(\mathbf{x}_{0} ; n\right)=\frac{1}{n} \ln \left\|\mathbf{D} \mathbf{F}^{n}\left(\mathbf{x}_{0}\right) \cdot \mathbf{v}_{k}\right\|
$$

where $k=1,2, \ldots, d_{H}$ and $\mathbf{v}_{k}$ is the singular vector related to $\xi_{k}\left(\mathbf{x}_{0}, n\right)$. The infinite-time limit of the above expression is the usual Lyapunov exponent $\lambda_{k}$ $=\lim _{n \rightarrow \infty} \tilde{\lambda}_{k}\left(\mathbf{x}_{0}, n\right)$. Although the time- $n$ exponent $\tilde{\lambda}_{k}\left(\mathbf{x}_{0}, n\right)$ generally takes on a different value, depending on the chosen point, its infinite-time limit takes on the same value for almost all $\mathbf{x}_{0}$ with respect to the natural ergodic measure of the invariant set $A$.

If the attractor in $A$ is chaotic (or hyperchaotic, in general), there are $d_{M}$ infinite-time Lyapunov exponents related to directions in the invariant subspace $M$, of which many can be positive; and $d_{H}-d_{M}$ Lyapunov exponents along directions transverse to $M$. For the sake of determining the transverse stability of $M$ it suffices to consider the largest one, $\lambda_{\perp}$. Hence condition 4 implies that $\lambda_{\perp}$ takes on a negative value:

$$
\lambda_{\perp}=\lim _{n \rightarrow \infty} \tilde{\lambda}_{\perp}\left(\mathbf{x}_{0}, n\right)<0 .
$$

The possible existence of an infinite number of transversely unstable orbits embedded in a transversely stable attractor implies the fluctuation of the finite-time largest transverse exponent, $\tilde{\lambda}_{\perp}\left(\mathbf{x}_{0}, n\right)$. Consider the probability distribution $P\left(\tilde{\lambda}_{\perp}\left(\mathbf{x}_{0}, n\right)\right)$, from which we can obtain the average value of this exponent (assuming proper normalization):

$$
\left\langle\tilde{\lambda}_{\perp}(n)\right\rangle=\int_{-\infty}^{+\infty} \tilde{\lambda}_{\perp}(n) P\left(\tilde{\lambda}_{\perp}(n)\right) d \tilde{\lambda}_{\perp}(n) .
$$

When $n$ is large enough the form of this distribution can be written as (Kostelich et al., 1997)

$$
P\left(\tilde{\lambda}_{\perp}(n), n\right) \approx \sqrt{\frac{n G^{\prime \prime}\left(\lambda_{\perp}\right)}{2 \pi}} e^{-n G\left(\tilde{\lambda}_{\perp}\right)},
$$

where the function $G(\lambda)$ has the following convexity properties:

$$
G\left(\lambda_{\perp}\right)=G^{\prime}\left(\lambda_{\perp}\right)=0, \quad G^{\prime \prime}\left(\lambda_{\perp}\right)>0 .
$$

Expanding $G(\lambda)$ in the vicinity of $\lambda_{\perp}$, the first nonvanishing term is the quadratic one, such that $P\left(\tilde{\lambda}_{\perp}(n)\right)$ reduces to a Gaussian distribution for $n \gg 1$ :

$$
P \approx \sqrt{\frac{n G^{\prime \prime}\left(\lambda_{\perp}\right)}{2 \pi}} \exp \left(-\frac{n G^{\prime \prime}\left(\lambda_{\perp}\right)}{2}\left[\tilde{\lambda}_{\perp}(n)-\lambda_{\perp}\right]^{2}\right),
$$

such that, on substituting into Eq. (25) the result is

$$
\left\langle\tilde{\lambda}_{\perp}\left(\mathbf{x}_{0}, n\right)\right\rangle=\lambda_{\perp} .
$$

Since the standard deviation in the Gaussian approximation approaches zero with $n^{-1 / 2}$, when $n \gg 1$, we can write the corresponding variance as

$$
\sigma^{2}=\left\langle\left[\tilde{\lambda}_{\perp}(n)-\left\langle\lambda_{\perp}\right\rangle\right]^{2}\right\rangle=2 D / n,
$$

such that the diffusion coefficient $D=1 / 2 G^{\prime \prime}\left(\lambda_{\perp}\right)$ is independent of $n$.

In terms of the distribution of finite-time transversal exponents, condition 5 for riddling implies that there is a positive fraction of positive values of $\tilde{\lambda}_{\perp}(n)$ for initial conditions $\mathbf{x}_{0}$ randomly chosen in the attractor $A$, i.e.,

$$
\phi(n)=\int_{0}^{\infty} P\left(\tilde{\lambda}_{\perp}(n)\right) d \tilde{\lambda}_{\perp}(n)>0 .
$$

Using Eq. (26), we find (Viana and Grebogi, 2001)

$$
\phi(n)=\frac{1}{2}+\frac{\sqrt{\pi}}{2} \operatorname{erf}\left(\lambda_{\perp} \sqrt{\frac{n}{4 D}}\right) .
$$

If the distribution of finite-time exponents has half of their values with positive sign, i.e., $\phi(n)=1 / 2$, then the infinite-time exponent $\lambda_{\perp}$ vanishes, and the invariant subspace $M$ loses transverse stability (a blowout bifurcation) (Ott and Sommerer, 1994; Yang, 2000).

\section{A simple example}

In order to illustrate the concept of a riddled basin, we begin with a simple example which is paradigmatic, for it exhibits the general aspects involved in the mathematical definition of riddling. We consider a noninvertible two-dimensional map F (Lai et al., 1996),

$$
\begin{aligned}
& x_{n+1}=4 x_{n}\left(1-x_{n}\right), \\
& y_{n+1}=p e^{-5\left(x_{n}-\chi\right)^{2}} y_{n}+y_{n}^{3},
\end{aligned}
$$

where $x \in[0,1],-\infty<y<+\infty$, and $p, \chi$ are positive parameters.

Since there are only odd powers of $y$ in Eq. (34) we have a $y \rightarrow-y$ symmetry, such that the condition $y=0$ defines an invariant subspace $M$ (condition 1) embedded in the two-dimensional phase space. In this case $y$ is the only transverse direction to $\mathrm{M}$. The $x$ dynamics is known to be chaotic, presenting a countable infinite number of unstable periodic orbits and a dense orbit on the attractor A (condition 2), from which we select $\chi=3 / 4$ as an unstable fixed point. The cubic term in the transverse dynamics implies that, if $\left|y_{n}\right|>1$, then $\left|y_{n+1}\right|>\left|y_{n}\right|>1$ for all further iterations of Eq. (34) such that once a trajectory reaches the line $|y|=1$ its $y$ values asymptote to infinity. We regard $|y|=\infty$ as the second attractor B outside the invariant subspace (condition 3 ).

The map (33) and (34) is simple enough to make possible an analysis of the onset of riddling in terms of a bifurcation of the unstable fixed point $\mathbf{x}_{P}:(x=3 / 4, y=0)$ of the attractor $A$, as $p$ is varied through a critical value $p_{c}$ (Lai et al., 1996). The eigenvalues of the Jacobian matrix, evaluated at $\mathbf{x}_{P}$, are $\xi_{\|}=-2$ and $\xi_{\perp}=p$, corresponding to eigendirections parallel and transverse to the invariant subspace $y=0$, respectively. It follows that $\mathbf{x}_{P}$, while always unstable along the invariant subspace, 

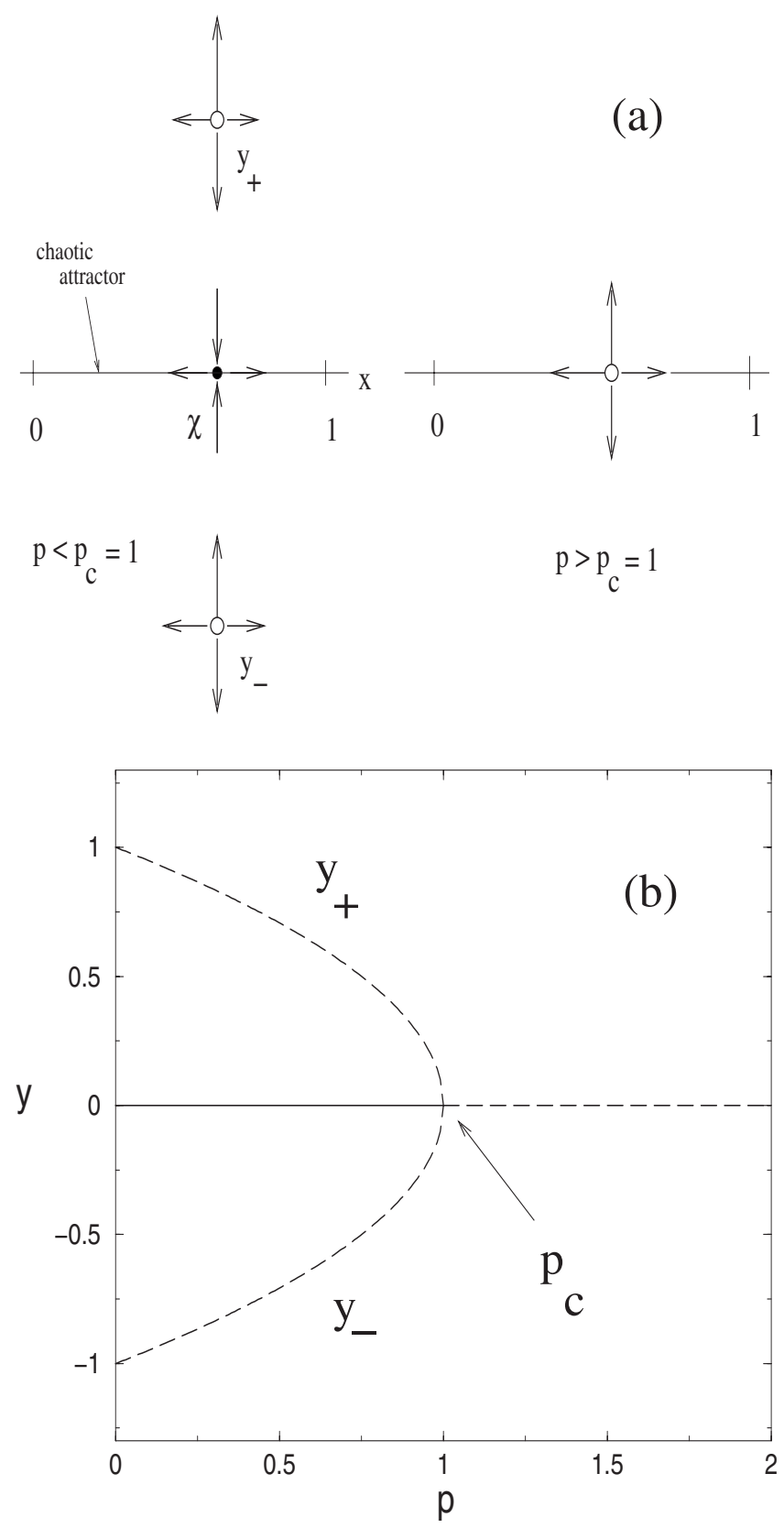

FIG. 17. Riddling bifurcation in the map of Eqs. (33) and (34). (a) Basic structure; (b) bifurcation diagram. From Viana and Grebogi, 2001.

is transversely stable (unstable) for $p<1(p>1)$. In other words, $\mathbf{x}_{P}$ can be a saddle or a repeller, if $p$ is, respectively, less than or greater than $p=p_{c}=1$.

In addition, the $y$ dynamics has also, for $p<1$, a pair of fixed points $\mathbf{r}_{ \pm}=\left(x=3 / 4, y_{ \pm}= \pm \sqrt{1-p}\right)$, and located off the invariant subspace [Fig. 17(a)]. The eigenvalues of the Jacobian matrix of the map when evaluated at these points are $\xi_{\|}=-2$ and $\xi_{\perp}=3-2 p$; they are always repellers. As a consequence, there is a saddle-repeller bifurcation at $p_{c}=1$ : for $p$ approaching $p_{c}$ from below, the saddle $\mathbf{x}_{P}$ and the pair of repellers $\mathbf{r}_{ \pm}$coalesce and, for $p$ greater than $p_{c}$, there remains only a repeller in the invariant subspace [Fig. 17(b)].

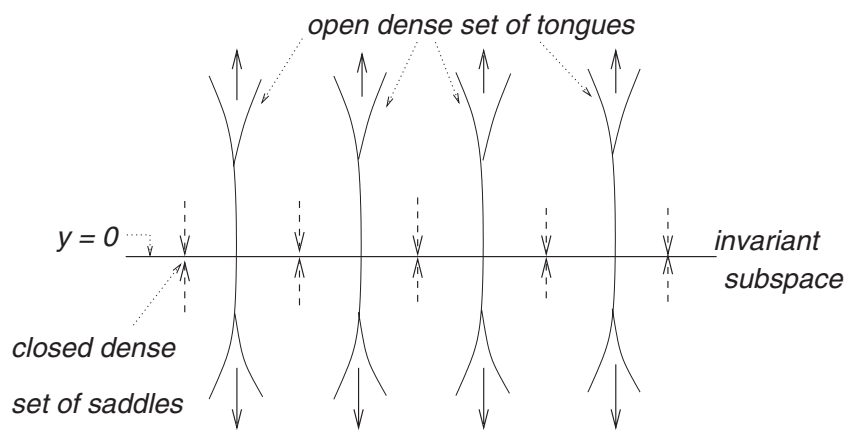

FIG. 18. Schematic showing the formation of tongues anchored at the invariant subspace when $p>1$. From Viana, Pinto, et al., 2003.

However, since the $x$ dynamics is noninvertible, not only the point $\mathbf{x}_{P}$ but all its infinite preimages become transversely unstable at the bifurcation $p=p_{c}$, forming an open and dense set of periodic points with Lebesgue measure zero (condition 5). The complement of this set is a Cantor-like closed and dense set of points which remain transversely stable, and carrying the whole Lebesgue measure of the $[0,1]$ interval. Since there is a dense chaotic orbit in $A$, a randomly chosen trajectory point will be transversely stable with unit probability (condition 4). The fixed points $\mathbf{r}_{ \pm}$belong to the boundary separating the basins of the attractor in the invariant subspace $M=[0,1]$ and the basin of the attractor at infinity. The boundary is a fractal curve for $p<p_{c}$ and there is no riddling at all. At the bifurcation point, however, this basin boundary collides with the attractor, the same occurring with all the countable infinite number of preimages of $\mathbf{x}_{P}$. The net result is the creation of an open dense set of extremely narrow tongues anchored at those preimages (Fig. 18).

To understand why these tongues are formed, we can consider the open set $|y|>1$ intersecting the transverse unstable subspace of $\mathbf{x}_{P}$. One of its preimages approaches $\mathbf{x}_{P}$ asymptotically, and these preimages form a subset of the tongue anchored at $\mathbf{x}_{P}$. Once a trajectory enters such a tongue it rapidly escapes to infinity, such that the open dense set of tongues belongs to the basin of the attractor at infinity. Hence the basin of $A$ is riddled with the tongues belonging to the basin of the infinity for $p \gtrless p_{c}=1$.

This can be inferred from Fig. 19(a), obtained for $p$ $=1.40$, and where the basin of infinity is represented by the black dots, which give a stalactitelike structure (Dronov and Ott, 2000). The basin of the chaotic attractor is densely filled with tongues of the other basin, but they are so narrow that they are barely seen due to finite graphic resolution. Figure 19(b) is a magnification near the fixed point $\mathbf{x}_{P}$, showing the self-similarity of the basin structure. The shape of the tongues is ultimately determined by the nonlinearity of the $y$ dynamics. (Lai et al. 1996) determined the boundaries of the main tongue at $\chi=3 / 4$ using a continuous-time approximation of the map equations near $\mathbf{x}_{P}$ and obtaining 

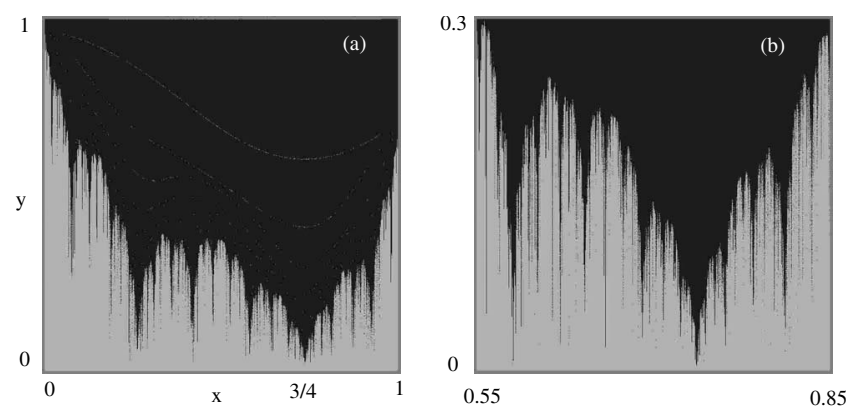

FIG. 19. Phase portraits for the map of Eqs. (33) and (34) for $p=1.40$. (a) Gray (black) dots belong to the basin of the chaotic (infinity) attractor. (b) Magnification of a region near the fixed point $\mathbf{x}_{P}$.

$$
|x-\chi|=\left(\frac{y}{\sqrt{(p-1)+y^{2}}}\right)^{1 /(p-1)},
$$

as long as $p \gtrsim p_{c}=1$. In this case, the invariant chaotic set A can be considered an attractor only in the weak Milnor sense, since any neighborhood of $A$ intersects, with positive Lebesgue measure, both its own basin and the basin of the infinity attractor.

It is also possible to verify the conditions 4 and 5 for riddling by analyzing the Lyapunov exponents along the directions parallel and transverse to the invariant subspace. Using Eq. (23) it follows that the time- $n$ exponents along the parallel and perpendicular directions are, respectively,

$$
\begin{aligned}
& \tilde{\lambda}_{\|}\left(x_{0}, y_{0}=0, n\right)=\frac{1}{n} \sum_{m=0}^{n-1} \ln \left|4\left(1-2 x_{m}\right)\right|, \\
& \tilde{\lambda}_{\perp}\left(x_{0}, y_{0}=0, n\right)=\ln p-\frac{5}{n} \sum_{m=0}^{n-1}\left(x_{m}-\frac{3}{4}\right)^{2}=\ln p-\mathcal{I}(n) .
\end{aligned}
$$

On the other hand, for typical chaotic trajectories in the attractor the natural invariant density on the attractor is $\rho(x)=1 / \pi \sqrt{x(1-x)}$, and the infinite-time counterparts of Eqs. (36) and (37) do not depend on the initial condition and thus can be obtained from Birkhoff's ergodic theorem as

$$
\begin{aligned}
& \lambda_{\|}=\lim _{n \rightarrow \infty} \tilde{\lambda}_{\|}\left(x_{0}, y_{0}=0, n\right)=\ln 2, \\
& \lambda_{\perp}=\lim _{n \rightarrow \infty} \tilde{\lambda}_{\perp}\left(x_{0}, y_{0}=0, n\right)=\ln p-\frac{15}{16},
\end{aligned}
$$

such that the chaotic attractor A loses transverse stability when $\lambda_{\perp}$ crosses zero, which occurs for $p=p^{*}$ $=\exp (15 / 16) \approx 2.55358 . \ldots$

Hence for $p_{c}=1<p<p *$ we have $\lambda_{\perp}<0$ for almost all initial conditions belonging to $A$, verifying condition 4 for riddling. On the other hand, the finite-time transverse exponent Eq. (37) can be positive even if its infinite-time counterpart is negative, provided $\ln p$ $>\mathcal{I}(n)>0$, which can occur for part of the initial condi-

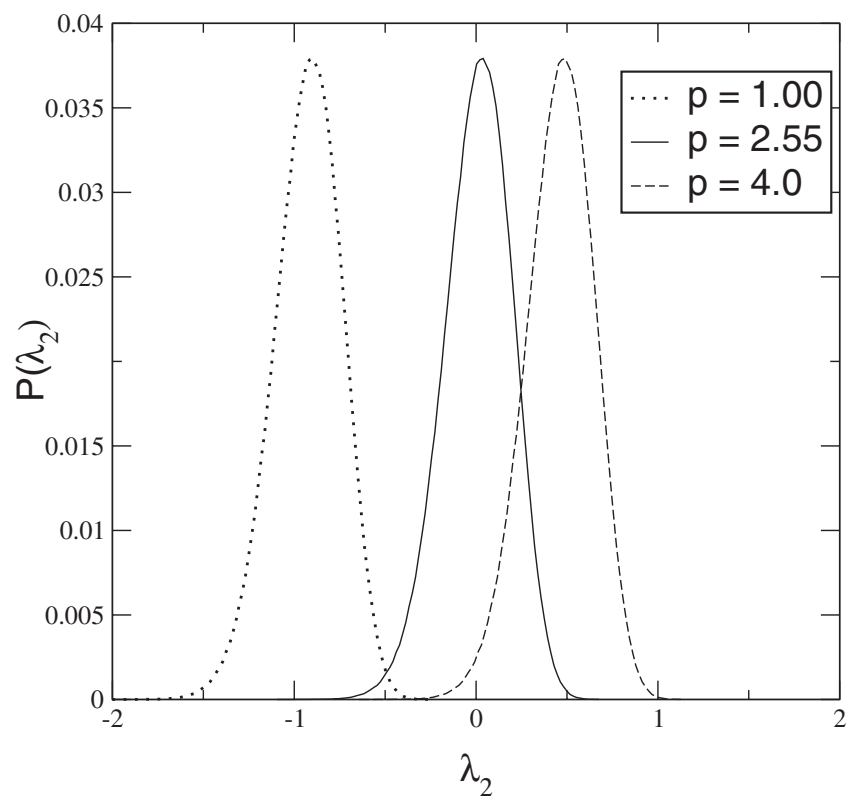

FIG. 20. Probability distributions for the transversal time-50 Lyapunov exponent and some values of the parameter $p$. From Viana, Pinto, et al., 2003.

tions $\left(x_{0}, y_{0}=0\right)$ chosen in the invariant subspace, since, depending on the value of $n$, the summation in $\mathcal{I}$ may assume different values as the trajectory approaches periodic orbits in $A$ which are transversely stable or unstable.

We can obtain a numerical approximation for the probability distribution of the finite-time transverse exponents, $P\left(\tilde{\lambda}_{\perp}(n)\right)$, by considering a large number of trajectories of length $n$ originating from initial conditions randomly chosen in the chaotic attractor A. In Fig. 20 we show distributions of time-50 exponents for values of $p$ before and after the bifurcation. Their shapes are indeed Gaussian-like, and the distributions drift toward positive values of $\lambda_{\perp}$, as $p$ increases past its critical value $p_{c}=1$. The variance of the average, with respect to a sample of size $n$, is a constant value, about 0.035 for all $p$ values, indicating that the Gaussian nature of the distribution $P\left(\tilde{\lambda}_{\perp}\right)$ is not significantly altered as $p$ is varied. Since the variance of the total population is the variance of the average multiplied by the sample size, the total variance of the time-50 exponents is 1.75 for the probability distributions depicted in Fig. 20, and Eq. (30) gives a diffusion coefficient of $D=0.875$.

We can also evaluate the fraction (31) of positive transverse time- $n$ exponents, shown in Fig. 21 as a function of $p$. For $p>1$ it is nonzero (condition 5) and increases monotonically for $p>1$, saturating at $\phi=1$ for large $p$. At the blowout bifurcation point $p^{*}$ we have $\phi$ $=1 / 2$, for exactly half of the time- $n$ exponents are positive. After this point the invariant subspace loses transverse stability, and the chaotic invariant set $A$ is no longer an attractor, even in the weak Milnor sense. There is no longer riddling, since A becomes a nonattracting chaotic saddle. Trajectories starting off but close 


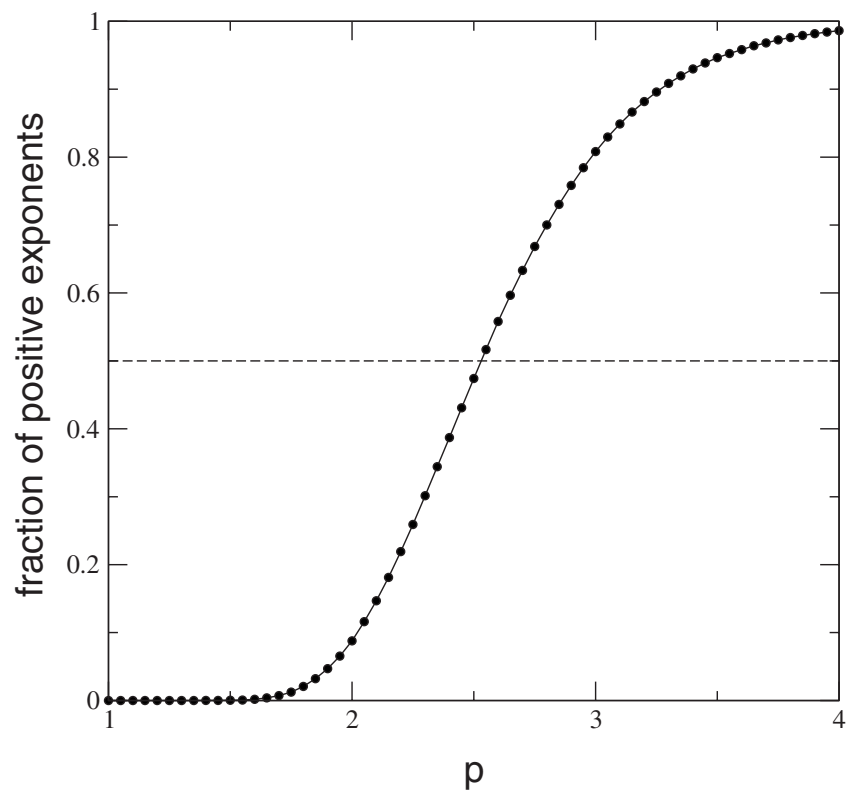

FIG. 21. Fraction of positive time-50 transversal Lyapunov exponents as a function of $p$, for the same parameters as in the previous figures. From Viana, Pinto, et al., 2003.

to this chaotic saddle will eventually diverge with time from the saddle, according to an exponential distribution of escape times. This fact can be exploited in defining exit or escape basins (Woltering and Markus, 1999).

\section{Scaling relations for riddling}

The riddling property can be illustrated by a numerical experiment (Ott et al., 1993, 1994) Consider a phase portrait just after the riddling bifurcation (see, e.g., Fig. 19), focusing on a horizontal line at $y=\ell$, and evaluate the fraction $V_{\ell}$ of its length that belongs to the basin of the chaotic attractor $A$. If the basin of $A$ is riddled with tongues belonging to the basin of infinity, it follows that for any distance $\ell$, no matter how small, there is always a nonzero value of $V_{\ell}$. Since the tongues are anchored at the invariant subspace $y=0$, this fraction tends to zero as $\ell \rightarrow 0$ (in the limit it is a Lebesgue measure zero set). Accordingly, the fraction of length belonging to the basin of the infinity is $P_{*}=1-V_{\ell}$, and it is expected to scale with $\ell$ as a power law,

$$
P_{*}(\ell) \sim|\ell|^{\eta}
$$

where $\eta>0$ is a characteristic scaling exponent. Figure 22 shows the results of Suetani and Horita (2001) for a parameter value $p>p_{c}$, showing that such a scaling law holds for $P_{*}$. Ott and co-workers have developed a stochastic model for explaining the fluctuations of the finite-time exponents, which predicts for this scaling exponent the following value (Ott et al., 1993, 1994):

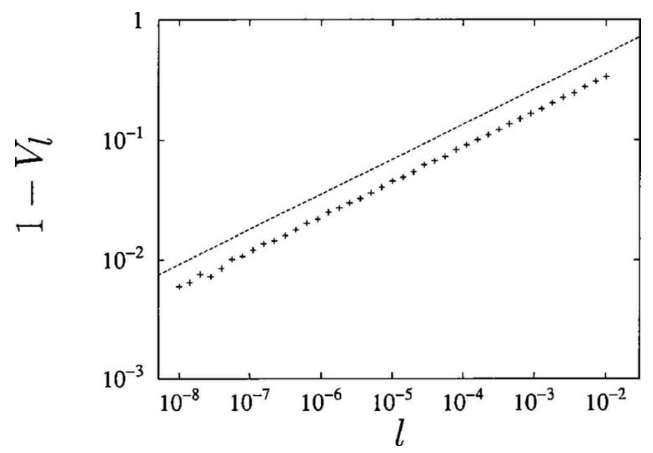

FIG. 22. Variation of the fraction $1-V_{\ell}$ with the distance from the invariant subspace when $p=2.092$. The solid line is a linear fit with slope $\eta=0.292$. From Suetani and Horita, 2001.

$$
\eta=\left|\lambda_{\perp}\right| / D,
$$

where $D$ is the diffusion coefficient obtained through the variance of the finite-time transversal Lyapunov exponent fluctuations.

The scaling law (40) conveys information on the measure of the basins of both attractors, but not about the arbitrarily fine-scaled riddling (Ott et al., 1993). In fact, the riddled basin of the chaotic attractor $A$ is a fat fractal, i.e., a fractal set with positive Lebesgue measure, whose fine-scale structure can be characterized by the so-called uncertainty exponent (Grebogi, McDonald, et al., 1983, 1985; McDonald et al., 1985); see Sec. III.A for more information on fat fractals. Consider again the line $y=\ell$ and choose randomly an initial condition $x_{0}$ on that line. Now choose randomly another initial condition $x_{0}^{\prime}$ with uniform probability within an interval of length $2 \epsilon$ and centered at $x_{0}$. If both points belong to different basins, they can be referred to as $\epsilon$ uncertain.

The fraction of $\epsilon$ uncertain points, denoted by $\langle p\rangle$, is the probability of making a mistake when attempting to predict which basin the initial condition $\left(x_{0}, y_{0}\right)=\ell$ is in, given a measurement uncertainty $\epsilon$, and is expected to scale with the latter as

$$
\langle p\rangle \sim \epsilon^{\varphi},
$$

where $\varphi \geqslant 0$ depends on both $x$ and $\ell$. It should be mentioned that $\langle p\rangle$ and $\varphi$ are the analogs of the uncertain fraction $f$ and uncertainty exponent $\alpha$, respectively, as defined in Sec. IV.C. The stochastic model of Ott and co-workers give the following expression in terms of the infinite-time Lyapunov exponents (Ott et al., 1993, 1994):

$$
\varphi=\lambda_{\perp}^{2} / 4 D \lambda_{\|} .
$$

Figure 23 presents the results of Suetani and Horita (2001) for $\langle p\rangle \epsilon$ for a given $\ell$ and several points on the invariant subspace, their results supporting the scaling relation (42) with an exponent $\varphi$ given by Eq. (43).

\section{E. A physical example}

The conditions for occurrence of riddling can be fulfilled by chaotic dynamical systems of physical interest. 


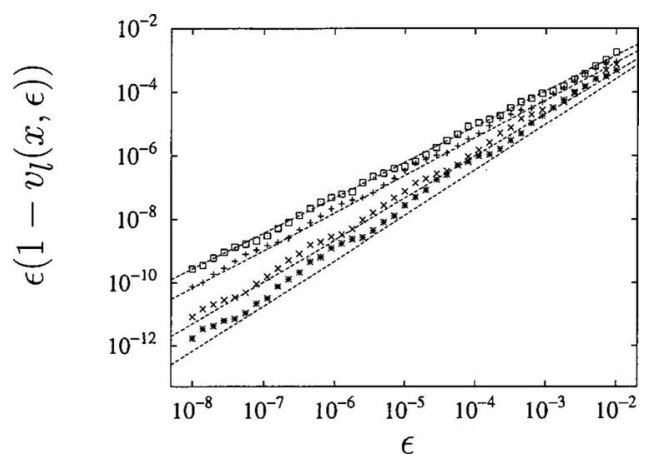

FIG. 23. Variation of the uncertain fraction (where $1-v_{\ell}$ of the figure is the same as $\langle p\rangle$ of the text) with $\epsilon$ for $\ell=0.01$ and various values of $x=\sin (\pi / 15), \sin (\pi / 31), \sin (5 \pi / 63)$, and $\sin (9 \pi / 127)$, corresponding to periodic points with periods 4,5 , 6 , and 7, respectively. The dotted lines are linear fits with slope given by Eq. (42) and $\eta=0.292$. From Suetani and Horita, 2001.

One example (Sommerer and Ott, 1993b; Ott et al., 1993, 1994; Sommerer, 1995) is a point particle of unit mass under the influence of a two-dimensional potential $V(x, y)$, viscous friction, and a time-periodic external forcing, whose equation of motion is

$$
\ddot{\mathbf{r}}+\nu \dot{\mathbf{r}}+\nabla V(\mathbf{r})=f_{0} \sin (\omega t) \hat{\mathbf{x}},
$$

where

$$
V(x, y)=\left(1-x^{2}\right)^{2}+(x+\bar{x}) y^{2}
$$

with $\nu, \bar{x}, f_{0}$, and $\omega$, respectively, the dissipation coefficient, a characteristic scale length, and the amplitude and frequency of the driving force.

The phase space $\mathrm{H}$ of this system is five dimensional, with coordinates $x, v_{x}, y, v_{y}$, and $\theta \equiv \omega t(2 \pi)$, in terms of which the equation of motion can be written as the autonomous vector field,

$$
\begin{aligned}
& \dot{x}=v_{x}, \\
& \dot{v}_{x}=-\nu v_{x}+4 x\left(1-x^{2}\right)-y^{2}+f_{0} \sin \theta, \\
& \dot{y}=v_{y}, \\
& \dot{v}_{y}=-\nu v_{y}+2 y(x+\bar{x}), \\
& \dot{\theta}=\omega .
\end{aligned}
$$

Due to the symmetry $V(x, y)=V(x,-y)$ the particle dynamics is invariant with respect to the $y \rightarrow-y$ and $v_{y} \rightarrow-v_{y}$ transformations in $\mathrm{H}$. Thus the constraints $y$ $=0$ and $v_{y}=0$ define a three-dimensional invariant subspace $\mathrm{M} \in \mathrm{H}$ in such a way that a trajectory with an initial condition $\left[x(0), y(0)=0, v_{x}(0), v_{y}(0)=0, \theta(0)\right]$ belonging to $M$ will remain there forever (condition 1). Restricted to this invariant subspace, the system state is described by the coordinates $\left(x, v_{x}, \theta\right)$, whose dynamics is governed by the equations of the forced double-well Duffing oscillator:

$$
\dot{x}=v_{x},
$$

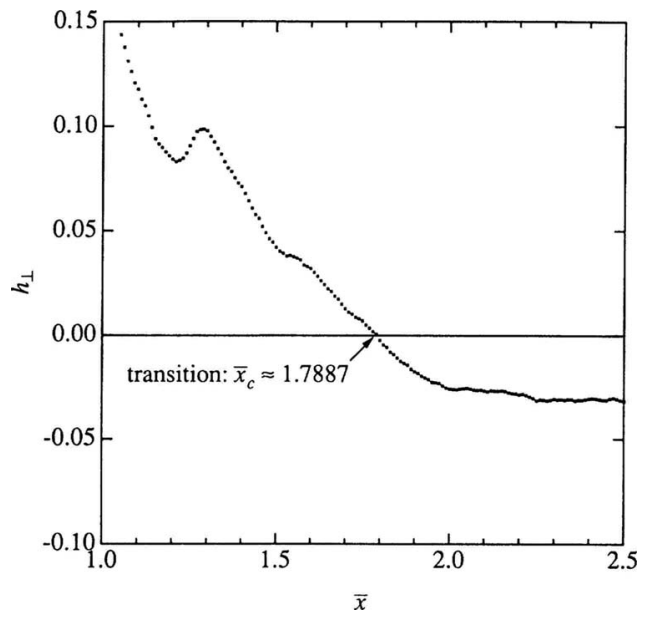

FIG. 24. Transverse Lyapunov exponent as a function of the control parameter $\bar{x}$, where $h_{\perp}$ of the figure is the same as $\lambda_{\perp}$ of the text. From Ott et al., 1993.

$$
\begin{aligned}
& \dot{v}_{x}=-\nu v_{x}+4 x\left(1-x^{2}\right)+f_{0} \sin \theta, \\
& \dot{\theta}=\omega,
\end{aligned}
$$

which has, for conveniently chosen values of its parameters, like $\nu=0.05, f_{0}=2.3$, and $\omega=3.5$, a chaotic attractor $A$ in the invariant subspace $M$ (condition 2). There is another attractor at infinity $\left(|x|=\infty,\left|v_{x}\right|=\infty\right)$ for the same set of parameters (condition 3).

The verification of the remaining conditions for riddling demands the computation of Lyapunov exponents in directions transverse to $M$. There are two such exponents, the largest one denoted $\lambda_{\perp}$, and which is computed by taking a variation of Eqs. (48) and (49) and setting $y=v_{y}=0$, which results in

$$
\begin{aligned}
& \dot{\delta} y=\delta v_{y}, \\
& \dot{\delta} v_{y}=-\nu \delta v_{y}-2[x(t)+\bar{x}] \delta y,
\end{aligned}
$$

where $x(t)$ is computed from a trajectory in the invariant subspace M, obtained by solving Eqs. (51)-(53), and which can be regarded as a driving term for Eq. (55). The largest finite-time transverse exponent, computed for the set of initial conditions $\mathbf{x}_{0} \equiv\{x(0), y(0)$ $\left.=0, v_{x}(0), v_{y}(0)=0, \theta(0)\right\}$, is then given by

$$
\tilde{\lambda}_{\perp}\left(\mathbf{x}_{0}, t\right)=\frac{1}{t} \ln \left[\frac{\delta(t)}{\delta(0)}\right],
$$

where

$$
\delta(t)=\sqrt{[\delta y(t)]^{2}+\left[\delta v_{y}(t)\right]^{2}}
$$

is the magnitude of a transverse displacement to the invariant subspace.

The infinite-time limit of Eq. (56), $\lambda_{\perp}$, determines the transverse stability of the Duffing attractor belonging to M. Figure 24 depicts its variation with the system parameter $\bar{x}$, showing that $\lambda_{\perp}$ takes on negative values for $\bar{x}$ $>\bar{x}_{c}=1.7887 \ldots$ (condition 4). Moreover, if the basin of 


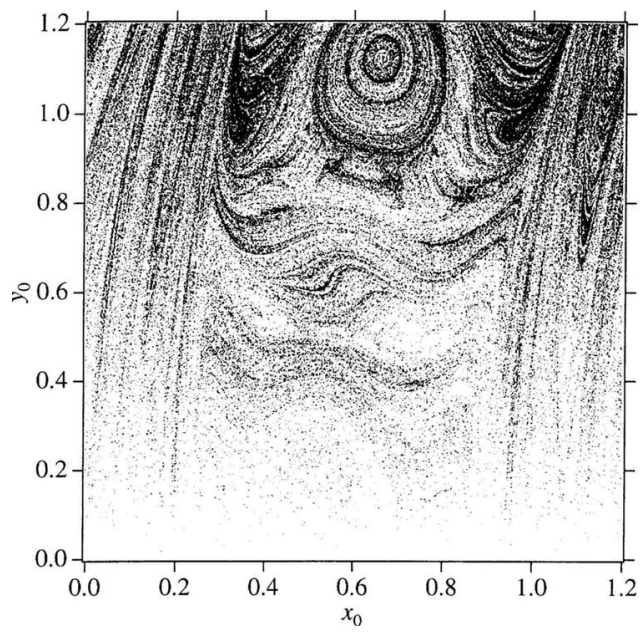

FIG. 25. Two-dimensional cross section of the phase space for the mechanical system given by Eqs. (46)-(50) and $\bar{x}=1.9>\bar{x}_{c}$. White (black) dots belong to the basin of the chaotic attractor (attractor at infinity). From Ott et al., 1993.

the Duffing attractor $A$ is riddled by the basin of the attractor at infinity, condition 5 implies that there are negative as well as positive fluctuations of $\tilde{\lambda}_{\perp}(t)$, in such a way that there is a nonzero fraction of positive finitetime Lyapunov exponents for $\bar{x}>\bar{x}_{c}$.

A glimpse of basin riddling in this system can be obtained from Fig. 25, where a two-dimensional cross section $v_{x}=\theta=0$ of the invariant subspace $\mathrm{M}$ shows points (represented by black dots) belonging to the basin of the infinity attractor $A$, with the blank regions in between representing points of the basin of the chaotic Duffing attractor. Both regions appear to be densely interwoven, which is confirmed by successive magnifications showing that any small region containing a blank area also contain black dots. The basin of infinity, however, is not riddled, since magnifications of a number of regions containing black points do not present blank areas (Ott et al., 1993).

We can perform the same numerical experiment as in the previous section, considering a horizontal line at $y$ $=\ell$ drawn in Fig. 25 and evaluating the fraction $P_{*}(1$ $-V_{\ell}$ ) of the length of that line lying in the basin of infinity, and expected to satisfy the scaling (40) with the distance $\ell$ to the invariant subspace, which intersects the plane depicted by Fig. 25 at the line $y=0$. Figure 26(a) shows the results of Ott et al. (1993), where the solid line represents a least squares fit with exponent $\eta$ $=0.5996 \pm 0.0064$, in good agreement with the theoretical prediction given by Eq. (41); see Fig. 10 of Ott et al., (1994).

Figure 26(b) shows the result of the second numerical experiment, which consisted of picking at random points on the $y=\ell$ line, considering points in intervals of length $2 \epsilon$ each and centered at such points, and evaluating the fraction $\langle p\rangle$ of points which are $\epsilon$ uncertain, i.e., the points within that interval which asymptote to different attractors. This fraction is nearly constant even though $\epsilon$
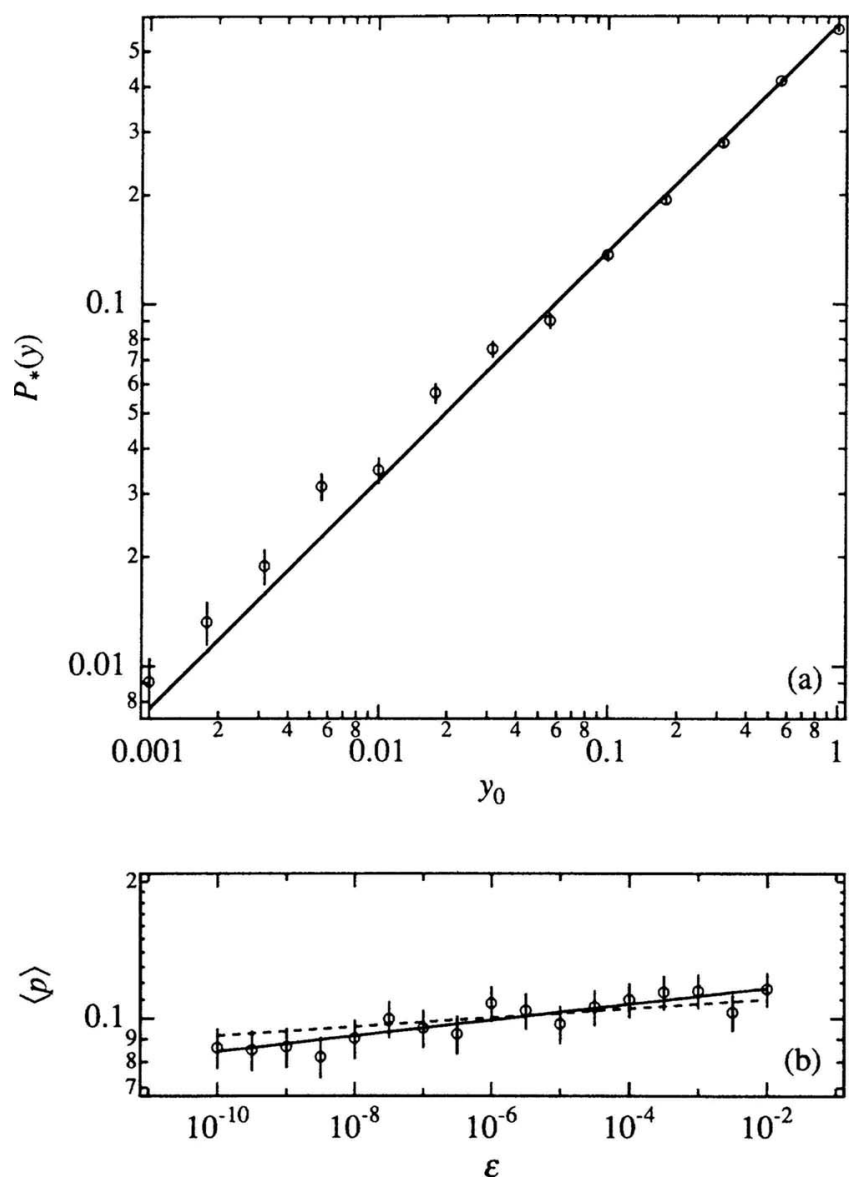

FIG. 26. Scaling laws for riddled basins. (a) Fraction of the length of a line $y=\ell$ which belongs to the basin of the infinity attractor, as a function of $\ell$, for $\bar{x}=1.85$; where $y_{0}$ of the figure is the same as $\ell$ of the text. The solid line is a least squares fit. (b) Fraction of $\epsilon$ uncertain points vs $\epsilon$, for $\bar{x}=1.90$. From Ott $e t$ al., 1993.

is varied over no less than eight orders of magnitude. The solid line is a least squares fit with exponent $\varphi$ $=0.0175 \pm 0.0038$, its smallness due to its quadratic dependence on $\lambda_{\perp}$ which is itself very small near the transition to a riddled basin (which occurs at $\bar{x}=\bar{x}_{c}=1.7887$ ).

The theoretical prediction, Eq. (43), gives for this exponent the value $\varphi=0.0093 \pm 0.0012$, the discrepancy with the numerical value being due to the use of a value $\bar{x}=1.9$ which is far from $\bar{x}_{c}$. A nearer value, on the other hand, would give values for $\varphi$ so small that a least squares fit would require exceptionally good statistics. The practical consequence of a near-zero exponent is that virtually no improvement in the precision of fixing the initial condition would diminish the probability of making a mistake when determining to which attractor that initial condition will asymptote to.

Unlike the simple example presented in the previous section, however, for this system we do not know in advance the bifurcation which actually leads to riddling, but presumably this is due to the loss of transverse stability of some low-period orbit embedded in the chaotic attractor. Moreover, this is typical of systems of physical interest. Therefore we have to use numerical diagnostics 
for characterization of riddling (in particular, the verification of condition 5). We have described two of them here: (i) the existence of the scaling law for the fraction of the basin of the second attractor $B$ when it riddles the basin of the chaotic attractor $A$; (ii) the computation of the finite-time Lyapunov exponents along the transversal directions in order to verify the existence of positive values. Both diagnostics, however, have their shortcomings.

As pointed out by Terry and Ashwin (2000), the scaling law (40) does not necessarily imply riddling, since any map with an unstable fixed point $P$ on an invariant subspace can display the same scaling if there are trajectories forming separatrices that divide the local unstable subspace of $\mathrm{P}$ into different regions according to whether points are asymptotic to an attractor inside or outside the invariant subspace. On the other hand, the mere existence of positive fluctuations of $\tilde{\lambda}_{\perp}(t)$ may also occur in other contexts, like the proximity of a near tangency of the stable and unstable subspaces of a periodic saddle orbit (Dawson et al., 1994). We conclude that the characterization of riddling, in practice, relies on the combination of a number of numerical diagnostics as well as some knowledge of the periodic orbit structure of the system.

\section{F. Riddling and synchronization of chaos}

One of the physical situations where riddled basins play a key role in the understanding of dynamical properties is the synchronization of chaotic trajectories. For this reason, this issue deserves a separate discussion, which nonetheless relies heavily upon that has been developed so far for general dynamical systems.

\section{Synchronization of chaos}

Although a chaotic system exhibits extreme sensitivity to initial conditions, two or more coupled identical systems can synchronize their trajectories so that they have the same values of the dynamical variables (Fujisaka and Yamada, 1983; Pecora and Carroll, 1990). Amplitude synchronization has been observed both numerically and experimentally in a variety of systems of physical and biological interest, such as nonlinear circuits, lasers, heart cells, etc. (Pecora et al., 1997; Boccaletti et al., 2002; Pikovsky et al., 2003).

Consider, for example, $N$ identical coupled systems such as a chain of continuous-time oscillators, each of them characterized by the $D$-dimensional state vector $\mathbf{x}^{(i)}(t), i=1,2, \ldots, N$. They are completely synchronized if

$$
\mathbf{x}^{(1)}(t)=\mathbf{x}^{(2)}(t)=\cdots=\mathbf{x}^{(N)}(t) \equiv \mathbf{s}(t),
$$

representing a set of $N-1$ equations defining a synchronization subspace $\mathrm{S}$, which turns out to be a $D$-dimensional subset of the full $N D$-dimensional phase space. The existence of the synchronization subspace depends on the coupling properties of the system. The oscillator chain can be written in the form

$$
d \mathbf{x}^{(i)} / d t=\mathbf{F}\left(\mathbf{x}^{(i)}\right)+\sum_{j} g_{i j} \mathbf{H}_{c}\left(\mathbf{x}^{(j)}\right),
$$

where $\mathbf{F}$ is the vector field representing the uncoupled and identical oscillators, $\mathbf{H}_{c}$ is a coupling function, and $g_{i j}$ is a coupling matrix. Substituting the synchronized state (58) into Eq. (59), it results that it is a solution of the dynamical equations provided $\Sigma_{j} g_{i j}=0$ for all $i$ $=1,2, \ldots, N$. The same condition holds for a discretetime coupled map lattice of the form

$$
\mathbf{x}_{n+1}^{(i)}=\mathbf{F}\left(\mathbf{x}_{n}^{(i)}\right)+\sum_{j} g_{i j} \mathbf{H}_{c}\left(\mathbf{x}_{n}^{(j)}\right) .
$$

Hence if an initial condition is placed on the synchronization subspace $S$, the resulting trajectory always lies on $S$ for all further times, so that $S$ is also an invariant subspace. Even if the synchronization subspace $S$ does exist, this fact does not necessarily imply that the system will eventually achieve this synchronized state, since $S$ must also be stable under infinitesimal perturbations in directions transverse to it.

When a trajectory starts off near the synchronization subspace it may undergo a variety of behaviors: (i) It can asymptote to $\mathrm{S}$ and remain there forever; (ii) it can stay for some time in the vicinity of $S$ and suddenly be pushed away from it, then return after some time to its vicinity, and so on (intermittent synchronization); (iii) it can wander through the accessible phase-space region without approaching S, i.e., permanent nonsynchronized behavior; or (iv) it can asymptote to another stationary state like a periodic or chaotic attractor, or even diverge to infinity. In order to investigate which behavior the system will follow we pursue first a local approach, which consists in making a linear stability analysis of the synchronization subspace. Second, we must go beyond the linear approximation to discover what the dynamics can be for large times.

Consider first a simple example from Hasler and Maistrenko (1997) consisting of $N=2$ coupled onedimensional $(D=1)$ maps,

$$
\begin{aligned}
& x_{n+1}=T\left(x_{n}\right)+\frac{\varepsilon}{2}\left(y_{n}-x_{n}\right), \\
& y_{n+1}=T\left(y_{n}\right)+\frac{\varepsilon}{2}\left(x_{n}-y_{n}\right),
\end{aligned}
$$

where $T(x)=1-2|x-1 / 2|$ is a tent map, which is a noninvertible transformation of $[0,1]$ onto itself, and $\varepsilon$ is the coupling strength. A cautionary remark is necessary here: for $\varepsilon \neq 0$ the coupled system does not leave the unit square $[0,1] \times[0,1]$ invariant, hence we have to extend the domain of Eqs. (61) and (62) to the real axis, so as to omit hereafter the restrictions $x \geqslant 0$ and $x \leqslant 1$.

The synchronized state $x_{n}=y_{n}$ is a possible solution of Eqs. (61) and (62) and thus defines a synchronization subspace $S$ represented by the $45^{\circ}$ diagonal line segment in the two-dimensional phase space. If we take an initial condition on the diagonal line $x=y$ but outside the interval defining $S$, the resulting trajectory will asymptote to 
infinity. Hence although the coupled map leaves the whole diagonal line invariant, we restrict the meaning of synchronized trajectories to those inside the interval S. The uncoupled maps $(\varepsilon=0)$ have positive Lyapunov exponent, namely, $\lambda^{U}=\ln 2>0$. Once a trajectory reaches the synchronization subspace $\mathrm{S}$, its dynamics is governed by the tent map, which means that there can exist synchronized chaotic trajectories in S. Whether or not an initial condition $\left(x_{0}, y_{0}\right)$ will produce a trajectory which asymptotes to $S$ and eventually becomes synchronized depends on the transversal stability of S.

Thanks to the simplicity of the tent map (its slope is constant for almost all points of $[0,1]$, except for a Lebesgue measure zero set), we can analyze the transversal stability of the synchronization subspace by explicitly computing the Jacobian matrix of the coupled map system (61) and (62) whose eigenvalues are

$$
\xi_{\|}=\left\{\begin{array}{cl}
2 & \text { if } x \leqslant 1 / 2, \\
-2 & \text { if } x>1 / 2,
\end{array} \quad \xi_{\perp}=\left\{\begin{array}{cc}
2-\varepsilon & \text { if } x \leqslant 1 / 2, \\
-2-\varepsilon & \text { if } x>1 / 2
\end{array}\right.\right.
$$

corresponding to the directions parallel and transverse to the synchronization subspace, respectively.

\section{Local and global riddling}

Now we turn to the relation between synchronization of chaos and riddled basins. The fact that the synchronization subspace, if it exists, is invariant under the system dynamics already fulfills condition 1 for riddling. Moreover, if the coupled identical systems undergo chaotic motion, their synchronized state is also chaotic, i.e., there is a chaotic attractor $A$ embedded in the synchronization subspace $S$ (condition 2). For condition 3 be satisfied there must exist another attractor B off the synchronized subspace (global riddling). There are many situations, however, for which there is no such attractor, but the trajectories off $S$ spend an arbitrarily large time wandering through the available phase-space region before returning to $S$ (local riddling). In the latter case we think of this nonsynchronized transient regime as a second possible behavior and assign to it the properties formerly possessed by the second attractor B, namely, conditions 4 and 5 of riddling. Local riddling has been investigated with respect to coupled chaotic systems (Kapitaniak and Maistrenko, 1998, 1999; Kapitaniak et al., 1998; Maistrenko et al., 1998, 1999a, 1999b; Manscher et al., 1998; Kim and Lim, 2001).

In the case of two coupled tent maps, Eqs. (61) and (62), the transverse stability can be inferred from the transverse Lyapunov exponent, which using Eq. (63) is given by

$$
\lambda_{\perp}=\lim _{n \rightarrow \infty} \sum_{m=0}^{n-1} \ln \left|\xi_{\perp}\right|=\int_{0}^{1} \rho(x) \ln \left|\xi_{\perp}\right| d x,
$$

where we have used Birkhoff's ergodic theorem. The invariant natural density of the skew tent map is uniform $(\rho=1)$ so that

$$
\lambda_{\perp}=-\frac{1}{2} \ln \left|4-\varepsilon^{2}\right|,
$$

which, being independent of $x$ and $y$, should also hold for the finite-time version. Hence in this case the probability distribution of the finite-time transversal exponents is a singular one,

$$
P\left(\tilde{\lambda}_{\perp}(n)\right)=\delta\left(\tilde{\lambda}_{\perp}(n)-\lambda_{\perp}\right) .
$$

In order to verify condition 4 for riddling, we investigate which values of the coupling coefficient $(\epsilon)$ the transverse Lyapunov exponent is negative. From Eq. (65) it follows that this is true provided $\epsilon$ belongs to either one of the two intervals $r_{1}(\epsilon) \equiv[\sqrt{3}, \sqrt{5}]$ and $r_{2}(\epsilon)$ $\equiv[-\sqrt{5},-\sqrt{3}]$. Now we turn to condition 5 , which implies that a set of unstable periodic orbits immersed in the chaotic synchronized state loses transversal stability, which can be analyzed by considering first the fixed points of the coupled maps (61) and (62), which are $\mathbf{0}$ $=(0,0)$ and $\mathbf{P}=(2 / 3,2 / 3)$. From Eq. (63) the transverse eigenvalues of the Jacobian matrix evaluated at these points are $\xi_{\perp}(\mathbf{0})=2-\boldsymbol{\epsilon}$ and $\xi_{\perp}(\mathbf{P})=-2-\boldsymbol{\epsilon}$.

The fixed point $\mathbf{O}$ will be transversely stable if $\left|\xi_{\perp}(\mathbf{0})\right|<1$, which occurs for $1<\epsilon<3$, which contains the interval $r_{1}(\epsilon)$ of the previous condition. Similarly, $\mathbf{P}$ is transversely stable if $\left|\xi_{\perp}(\mathbf{P})\right|<1$, which implies $-3<\epsilon$ $<-1$, also containing the second interval $r_{2}(\epsilon)$. Regardless of $\epsilon$ being positive or negative, if condition 4 holds, at least one of the fixed points is transversely unstable, and so are all its preimages, since the map is noninvertible. The existence of a denumerable infinite number of periodic points which are transversely unstable verifies condition 5 for riddling provided $\epsilon \in\left\{r_{1}(\epsilon) \cup r_{2}(\epsilon)\right\}$.

The set of initial conditions in $\mathbb{R}^{2}$ that asymptotes to the chaotic attractor $A$ is called its basin of synchronization. Since $\lambda_{\perp}<0$ (condition 4), almost all synchronized trajectories are transversely attracting, and $\mathrm{A}$ is a weak Milnor attractor (Hasler and Maistrenko, 1997; Tan et al., 2003). The open dense set of transversely unstable points in A (condition 5) makes the basin of synchronization at least locally riddled: there is a neighborhood $U$ of $\mathrm{A}$ such that in any neighborhood $V$ of any point in $\mathrm{A}$ there is a set of points in $V \cap U$ of positive Lebesgue measure which leave $U$ in a finite time. In other words, starting from any point near the chaotic synchronized trajectory in $\mathrm{S}$, there is a nonzero probability that a trajectory is pushed away from $\mathrm{S}$. A schematic picture is provided by the top panel of Fig. 27, where the unit square $[0,1] \times[0,1]$ is represented with the corresponding synchronization subspace depicted as the bold segment lying on the dotted line. The sets $U$ and $V$ have a nonempty intersection, which contains another set with points generating orbits that eventually leave $U$, one of them represented by a curved arrow.

The basic mechanism by which those nonsynchronized trajectories are repelled from $S$ is the same as in the two-dimensional map previously discussed. Local riddling in this case implies that there is a neighborhood $U$ of $\mathrm{S}$ and a set $V$ of tongues anchored at the trans- 


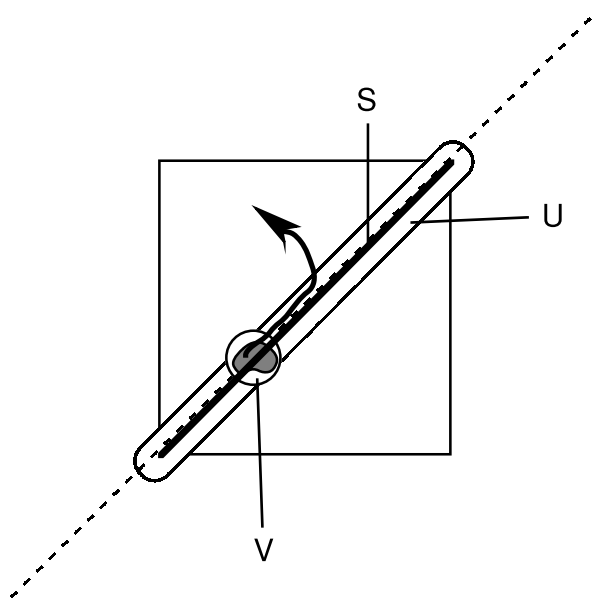

(a)

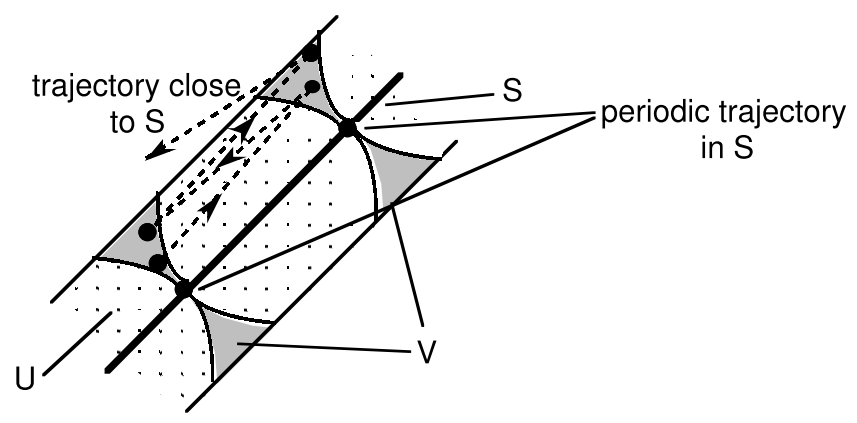

(b)

FIG. 27. Locally riddled attractor for two coupled tent maps. Top: Scheme showing the sets involved in the definition of the local riddling (see text). Bottom: Tonguelike structures emanating from a transversely unstable orbit embedded in the synchronization subspace $\mathrm{S}$. Trajectories close to $\mathrm{S}$ are ejected away from S, after entering such tongues. From Hasler and Maistrenko, 1997.

versely unstable points in the synchronization subspace (see the bottom panel in Fig. 27). Any trajectory off but close to $\mathrm{S}$ that falls into $V$ can leave $V$ only through the boundary of $U$ (Hasler and Maistrenko, 1997). Since the preimages of the transversely unstable points are dense in $\mathrm{S}$, there is always a nonzero probability that the trajectory will be repelled from $S$ through one of these tongues.

The fate of the trajectories off the synchronization subspace cannot be told only from a local stability analysis of periodic orbits in $S$ and thus requires a global analysis of the transverse dynamics. If there are attractors other than the synchronized one, a trajectory which starts from an initial condition off but close to $S$ will stay in its vicinity for a while, yielding a nearly synchronized trajectory, until it enters a tongue and is repelled from S, which generates a bursting nonsynchronized trajectory. If the basin of synchronization is locally riddled, such trajectories leaving the neighborhood of S eventually return to it, generating another "laminar" region of synchronization and so on. This intermittent switching between synchronized and nonsynchronized behavior has been observed in a variety of other systems (Viana, Grebogi, et al., 2003, 2005).
By contrast, suppose that in the coupled system there is a second attractor $B$, such that the basin of the synchronized attractor is riddled with tongues belonging to the basin of B, or global riddling. Tan et al. (2003) performed a global analysis of two coupled skew-tent maps, for which our Eqs. (61) and (62) follow as particular cases [note that the coupling constant in Tan et al. (2003) differs from ours by an unessential factor of 2]. For the latter, the results of Tan et al. (2003) imply that the coupling parameter values corresponding to locally riddled basins are the isolated values $\varepsilon=2$ and $\varepsilon=-2$. Moreover, if we consider the attractor at infinity as the attractor B off the invariant subspace, it is possible to observe global riddling. Accordingly, the coupling parameter interval for which global riddling takes place was found to be (i) $\varepsilon \in r_{1}(\epsilon)$ and $\varepsilon \neq 2$, and (ii) $\varepsilon \in r_{2}(\varepsilon)$ and $\varepsilon \neq-2$, which is a dense set of values of $\varepsilon$, thus structurally stable with respect to small parameter variations due to noise, for example.

\section{G. Riddledlike basins}

The mathematical conditions for riddling, as introduced in Sec. VIII.B, are difficult to verify directly in dynamical systems of physical interest. Conditions 1-3 are the easiest to fulfill, whereas 4 and 5 depend on the knowledge of the periodic orbit which becomes transversely unstable, while the invariant subspace itself remains transversely stable. This analysis is feasible only in low-dimensional examples, as we have seen. Normally, when faced with dynamical systems of higher dimensionality, one has to resort to other numerical diagnostics of riddling, like finite-time transversal Lyapunov exponents and scaling relations. In general, we can think of any of the five conditions to be broken in some situation, and many alternative concepts have appeared in recent years, which can be gathered together in the general category of riddledlike basins.

\section{Catastrophe of riddling}

Condition 1 for riddling requires the existence of an invariant subspace of the dynamical system. If this subspace is a geometrical consequence of a given symmetry of the system, once this symmetry is broken by some means-as extrinsic noise, for example-there is no longer an invariant subspace and we cannot have, strictly speaking, riddled basins. Lai and Grebogi studied the loss of the riddling property as the dynamical system loses its invariant subspace, calling it the catastrophe of riddling (Lai and Grebogi, 2000). In this case, a symmetry-breaking perturbation can replace riddled basins by fractal ones. The mathematical reason is the presence of open sets following the shift of the periodic orbit locations due to the symmetry-breaking perturbation. From the physical point of view, however, many consequences of riddling can still be observed. For example, even though a riddled basin becomes fractal, it may lead to a similar type of extreme sensitivity of the 
(a) $\varepsilon=0$

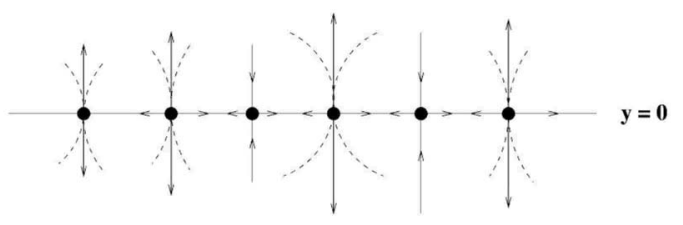

(b) $|\varepsilon| \sim 0$

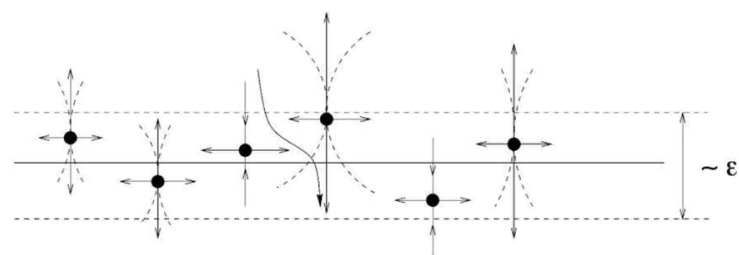

- attractor

FIG. 28. Schematic of the transversal dynamics for (a) $\epsilon=0$ (riddled basins) and (b) $|\epsilon| \approx 0$ (fractal basins). From Lai, 2000.

asymptotic attractor on initial conditions to that caused by riddled basins (Lai and Andrade, 2001).

In order to illustrate these points, we return to the two-dimensional map used in Sec. VIII.C, adding a symmetry-breaking parameter $\epsilon$ to the $y$ dynamics:

$$
\begin{aligned}
& x_{n+1}=r x_{n}\left(1-x_{n}\right), \\
& y_{n+1}=\epsilon+p e^{-5\left(x_{n}-\chi\right)^{2}} y_{n}+y_{n}^{3},
\end{aligned}
$$

where $x \in[0,1], r=4, \chi=3 / 4$, and $-\infty<y<+\infty$ (Viana and Grebogi, 2001). If $\epsilon=0$, we have a perfect $y \rightarrow-y$ symmetry and thus $y=0$ is an invariant subspace $\mathrm{S}$, on which there is a chaotic attractor. In this case, we have seen that there is another attractor at $y=+\infty$. By symmetry there is yet another one at $y=-\infty$. For $p<p_{c}=1$ the chaotic attractor is transversely stable and all periodic orbits embedded in it are transversely stable.

After $p_{c}=1$ (riddling bifurcation) the fixed point ( $x^{*}$ $=3 / 4, y^{*}=0$ ) and all its preimages become transversely unstable, while the chaotic attractor is still transversely stable. The periodic points become the roots of a set of tongues anchored at the invariant subspace. Tongues with positive or negative values of $y$ belong to the basin of the attractor at $y= \pm \infty$, respectively. The roots of these tongues are dense in $S$ and have Lebesgue measure zero, its complement assuming the full measure in $\mathrm{S}$. By continuity, in the vicinity of $S$ the complement of the set of the tongues, which is the basin of the chaotic attractor in S, has a positive Lebesgue measure [Fig. 28(a)]. Hence the basin of the chaotic attractor is riddled with measure-theoretic holes, but with a positive Lebesgue measure, and contains no open sets.

Now we consider a nonzero value for $\epsilon$, thus destroying the invariance of the subspace $S$. If $\epsilon$ is small enough, we expect that the periodic orbits in $S$ will not change their stability properties, i.e., if they are transversely stable (or unstable) when $\epsilon=0$, they will remain so for $\epsilon \neq 0$. However, it turns out that the locations of such orbits shift under this perturbation, but nevertheless they will be confined within a strip of width $\sim \epsilon$ centered at $y=0$ [Fig. 28(b)]. In particular, all transversely unstable repellers will be shifted upwards $(y>0)$, whereas all transversely stable saddles will be shifted downwards $(y<0)$. As a consequence, a trajectory starting from an initial condition placed in $y>0$ can move across the $x$ axis and asymptote to the attractor at $y=$ $-\infty$. Hence the basin of $y=-\infty$ acquires points with positive $y$. There is evidently still an open set with $y>0$ which asymptotes to $y=+\infty$, but it is no longer dense. The boundary between the basins of $+\infty$ and $-\infty$ is a fractal set, and the formerly riddled basins have changed into fractal basins, irrespective of how small $\epsilon$ may be.

In Sec. VIII.C we have also seen that, for $\epsilon=0$, as the parameter $p$ is increased beyond a second critical value $p^{*} \approx 2.55358 \ldots$, the formerly chaotic attractor at the invariant subspace $S$ loses transversal stability as a whole, and it is no longer an attractor. When $\epsilon \neq 0$, the metamorphosis arisen when a riddled basin becomes a fractal one is followed by the creation of chaotic transients. If $p<p^{*}$, the transient lifetimes scale with the parameter difference as

$$
\tau \sim \exp \left[C(\epsilon)\left(p^{*}-p\right)|\ln \epsilon|\right] \quad\left(p<p^{*}\right)
$$

where $C(\epsilon)>0$. When $p>p^{*}$, the transient lifetime is much shorter than that yielded by this scaling law (Lai and Grebogi, 2000). Lai and Andrade (2001) worked out the stochastic model of Ott et al. (see Sec. VIII.D) and obtained scaling relations for the symmetry-broken case.

Moreover, the uncertainty dimension $d$ of the fractal basin boundary which results from the catastrophe of riddling is close to that of the phase-space dimension $D$ itself. This is characteristic of an extreme form of final state sensitivity which has formerly been described in systems of coupled oscillators (Lai, 1995), and makes predictability in these systems even harder than if they showed fractal or Wada basins. In terms of the uncertain fraction of the phase space, which scales as a power law with the uncertainty radius as $\epsilon^{\alpha}$, where $\alpha=D-d$ is the uncertainty exponent (Grebogi, McDonald, et al., 1983; McDonald et al., 1985), we thus have $D \approx d$ and $\alpha \approx 0$ after the catastrophe of riddling. In practical applications, we can regard $\epsilon$ as coming from the extrinsic (and unavoidable) noise. Moreover, in coupled oscillator systems, the invariant subspace can be viewed as the synchronization subspace only if the coupled systems are identical, which is extremely difficult to ensure. Hence the catastrophe of riddling is quite common in mechanical systems but, since the observable consequences are similar to riddled basins, these cases have been treated as a kind of practical riddling (Blazejczyk-Okolewska et al., 2000; Kapitaniak, 2001). 


\section{Pseudoriddling}

Our condition 2 for riddling is that the system possesses an invariant subspace with a chaotic attractor therein. The key point on having a chaotic attractor is the existence of an infinite number of unstable periodic points embedded on it, such that many of them can become transversely unstable without changing the overall transversal stability of the chaotic attractor itself. Lai (2001) argued that such condition is only sufficient but not necessary, and can be replaced by the weaker requirement that a chaotic invariant set must exist in the invariant subspace $S$. In such cases, if the remaining conditions are satisfied, one would have pseudoriddling.

A chaotic attractor is obviously such an invariant set, but it would also be a nonattracting chaotic set. Such sets are common in dynamical systems since they may occur as a result of sudden changes on chaotic attractors caused by arbitrarily small parameter changes; see Sec. IV.D for more information. A well-known example is the existence of an infinite number of periodic windows embedded into the mainly chaotic region of the bifurcation diagram of a unimodal map like the logistic map $x_{n+1}=a x_{n}\left(1-x_{n}\right)$ for $a>a_{\infty}=3.569945672 \ldots$ (Gulick, 1992). At any given periodic window there is the coexistence of an attracting periodic orbit and a nonattracting chaotic set. For the period-3 window starting at $a$ $=a_{3}=1+\sqrt{8}=3.828427 \ldots$, we have a coexistent nonattracting chaotic set described by Li and Yorke (1975). In terms of the weakened conditions for riddling introduced by Lai, one can analyze the dynamics where there is a nonattracting chaotic set in an invariant subspace $\mathrm{S}$ and an attracting periodic orbit off the invariant subspace. This may be achieved with the two-dimensional prototype (67) and (68), provided $a$ be chosen to be within a period-3 window of the logistic map $\left(a \gtrsim a_{3}\right)$. It has been found that pseudoriddling can occur in the transverse vicinity of a nonattracting chaotic set, with scaling laws similar to those described for riddled basins of attraction of chaotic attractors (Lai and Grebogi, 1999; Terry and Ashwin, 2000; Lai and Grebogi, 2000).

\section{EXPERIMENTAL VERIFICATION OF FRACTAL BASINS}

Since the appearance of fractality and uncertainty associated with nonlinear dynamics, many experimental verifications of the existence of fractal structures have been carried out. Most of this work has been focused on chaotic attractors and their fractal dimension. The reason is that chaotic attractors are invariant attracting structures, and therefore it is easy to reproduce them in many real systems.

The literature on the experimental realization of fractal basin boundaries, on the contrary, is surprisingly short. The reason is the extreme difficulty of developing in a real system the typical numerical technique, that is, the division of phase space in a fine grid of initial conditions and their iteration until a final state is reached (Sweet and Ott, 2000a, 2000b). In general, it is not pos- sible to prepare the initial conditions with such precision or such density that the fine fractal scale is observable. Furthermore, many experiments are so long that cannot be repeated the number of times that is needed to plot a basin diagram, and actually in many cases each reproduction of the experiment is influenced by the former one. This promising field of experimental nonlinear dynamics started when Heagy et al. (1994b) were able to follow the present methodology and proved experimentally the existence of riddling in an electrical circuit. It is in some sense ironic that the strangest fractal structure, and one of the latest to be discovered, was the first one to be seen in nature. One year later, Cusumano and Kimble (1995), following the work by Heagy et al., overcame the disadvantage of needing a fine grid of initial conditions, and developed a general procedure for dissipative mechanical systems. After these two seminal papers on the subject, other methods that simplify the experimental realizations have been developed, and the search for real fractal basin boundaries has mainly been focused on fields where fractality can be enjoyed with the naked eye, such as fluids or optical devices.

In this section, we follow a historical order to present the different experimental works, as each step in this difficult task has been deeply influenced by the earlier ones. We have divided the examples in two main groups, according to the fields that have shown to be more promising for the visualization of these structures: riddled basins in electrical circuits, and fractal and Wada basins associated with chaotic scattering systems.

\section{A. Riddled basins in electrical circuits}

The first laboratory observation of a phenomenon identified with riddled basins was by Ashwin et al. (1994), who observed attractor bubbling near the synchronization threshold of two coupled chaotic circuits. However, the first direct experimental evidence of riddling was by Heagy et al. (1994b), who investigated global riddling related to the synchronization subspace of a system composed of four Rössler-like circuits with diffusive coupling. Moreover, they compared their findings with numerical simulations from the circuit equations, both approaches giving similar results for the scaling typical of riddling.

The circuits used are described in Heagy et al. (1994a) and their equations constitute a modified version of the Rössler equations, where the quadratic nonlinearity is replaced by a piecewise linear element. Each circuit was capable of exhibiting chaotic behavior over a large range of parameters, so that weak coupling to other circuits would not immediately destroy the chaotic behavior. The configuration consisted of four identical such circuits arranged in a ring, and coupled diffusively to the nearest neighbors of each other through one of their variables.

The equations describing the system are, in nondimensional form,

$$
\dot{x}_{i}=-f y_{i}-z_{i}-r x_{i}+k\left(x_{i+1}+x_{i-1}-2 x_{i}\right),
$$




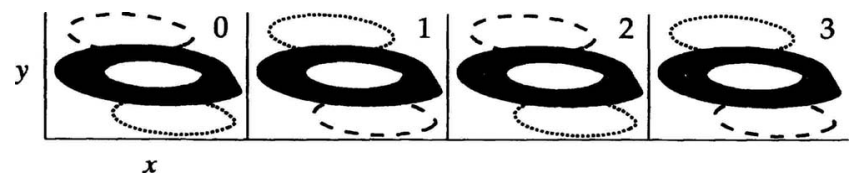

FIG. 29. Projection, in the $x-y$ plane, of the coexisting attractors (solid line, synchronized chaotic attractor, dashed and dotted lines, nonsynchronized period-1 attractors) of four coupled Rössler-like systems for $k=0.935$. From Heagy et al., $1994 \mathrm{~b}$.

$$
\begin{aligned}
& \dot{y}_{i}=x_{i}+a y_{i}, \\
& \dot{z}_{i}=-b z_{i}-+\operatorname{cg}\left(x_{i}\right),
\end{aligned}
$$

where $i=1, \ldots, 4$, and $g(x)$ is piecewise linear, given by

$$
g(x)= \begin{cases}0 & \text { if } x \leqslant d \\ x-d & \text { if } x>d,\end{cases}
$$

and periodic boundary conditions are assumed. The parameters were set as $a=0.13, b=1.0, c=15.0, d=3.0, f$ $=0.5$, and $r=0.05$; the control parameter $k$, can vary from 0.0 to 1.0 .

Heagy et al. (1994a) found a stable synchronous chaotic state for coupling values $0.031<k<0.945$ in the model and $0.08<k<0.82$ in the circuit. In the vicinity of a bifurcation value $k=0.945$ there are also two other period-1 attractors off the synchronization subspace. This bifurcation corresponded to the onset of a shortwavelength instability that shows up as bursts taking the overall system to the periodic attractors. Figure 29 shows a projection of the three coexisting attractors for $k=0.935$, which corresponds, in the experimental setting, to the value $k_{\mathrm{EXP}}=0.805$. The phase space of this system is 12-dimensional, and the three-dimensional synchronization subspace is the hyperplane $\mathrm{S}$ defined by the conditions $x_{1}=x_{2}=x_{3}=x_{4}=x_{s}, y_{1}=y_{2}=y_{3}=y_{4}=y_{s}$, and $z_{1}=z_{2}$ $=z_{3}=z_{4}=z_{s}$. The subspace $\mathrm{S}$ is defined invariant under the system dynamics given by Eqs. (70)-(72) (condition 1 for riddling) and contains a synchronized chaotic attractor for reasonably large intervals of the system parameters (condition 2), as well as nonchaotic attractors off $\mathbf{S}$ (condition 3).

For ease of visualization, a two-dimensional grid of initial conditions was conveniently chosen so that the horizontal coordinate $(\Delta x)$ stands for the synchronization subspace, the vertical one $(\Delta y)$ in the transverse direction. All $z$ variables were set to the same value $z_{s}$ for all initial conditions. The horizontal direction was chosen to be the deviation $\Delta x$ of the initial $x$ value from $x_{s}$ for all oscillators. The vertical direction, however, was chosen to have a pure transverse Fourier mode by setting $y_{i}=y_{s}+(-1)^{i} \Delta y$, where $i=0,1,2,3$. For each grid point the circuit variables were evolved through a given time $N_{1}$ to let transients die out, and the Fourier mode coefficient $S_{2}=y_{0}-y_{1}+y_{2}-y_{3}$ was then computed and averaged over a time interval $N_{2}$. Here $N_{1} \approx 1000$ and $N_{2}$ $=100$ are taken in number of cycles around the Rösslerlike attractor.

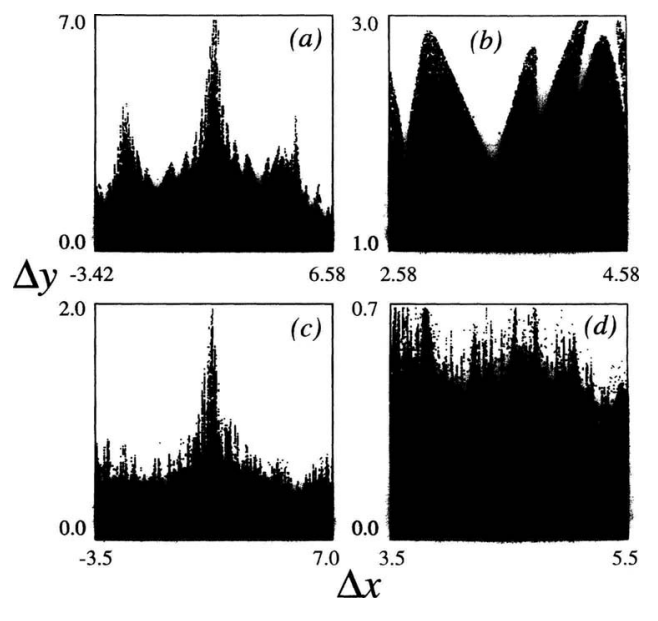

FIG. 30. Basins of attraction for coupled Rössler-like systems in the $\Delta x-\Delta y$ plane. Black, basin of synchronized chaotic attractor; white, basin of period-1 attractor, obtained by (a) experiment, (c) numerical integration. (b), (d) Magnifications of regions belonging to (a) and (c), respectively. From Heagy et al., 1994b.

If the average value of $S_{2}$ falls below some threshold, it was assumed that the circuit reached the synchronized state $S$ and a black dot was plotted at the grid point. Otherwise, no dot was plotted and the system asymptotes to one of the two periodic (according to the sign of $\left.\left\langle S_{2}\right\rangle\right)$ attractors. Figure 30 shows the results of Heagy et al. (1994b) for a $200 \times 200$ grid of initial conditions and only one of the periodic attractors. The basin of the synchronous attractor (black dots) is indeed riddled, a fact confirmed both experimentally [Fig. 30(a) with a magnification in Fig. 30(b)] and numerically [Figs. 30(c) and 30(d)].

Conditions 4 and 5 for riddling cannot be verified directly for this example, since the dimensionality of the phase space is too high Kapitaniak et al. (2003). However, we can get indirect evidence for riddling by verifying the scaling relations typical of riddled basins. One of them involves the measure of a line length of a given transverse distance to the synchronized subspace, which we denote as $P_{*}$ (see Sec. VIII.D), and which turns here to be the probability of going to the period-1 (nonsynchronized) attractor, as a function of the mode-2 Fourier amplitude $\Delta y$, which plays the role of the transverse distance [see Figs. 31(a) and 31(b)]. It has been verified that $P_{*}$ scales with this distance as $|\Delta y|^{\eta}$, in accordance with Eq. (40), with exponents $\eta=2.03 \pm 0.08$ and $2.06 \pm 0.08$ for experimental data and numerical simulations, respectively. One of the reasons behind these slightly different results lies in the behavior of the phase-space trajectories near the synchronization subspace, for the probability of going to the periodic attractor levels off due to noise effects on the experimental apparatus.

\section{B. Fractal basins in chaotic scattering}

\section{Hydrodynamical flows}

It is of general knowledge that a mixture of different fluids can give rise to very complex patterns, but what 

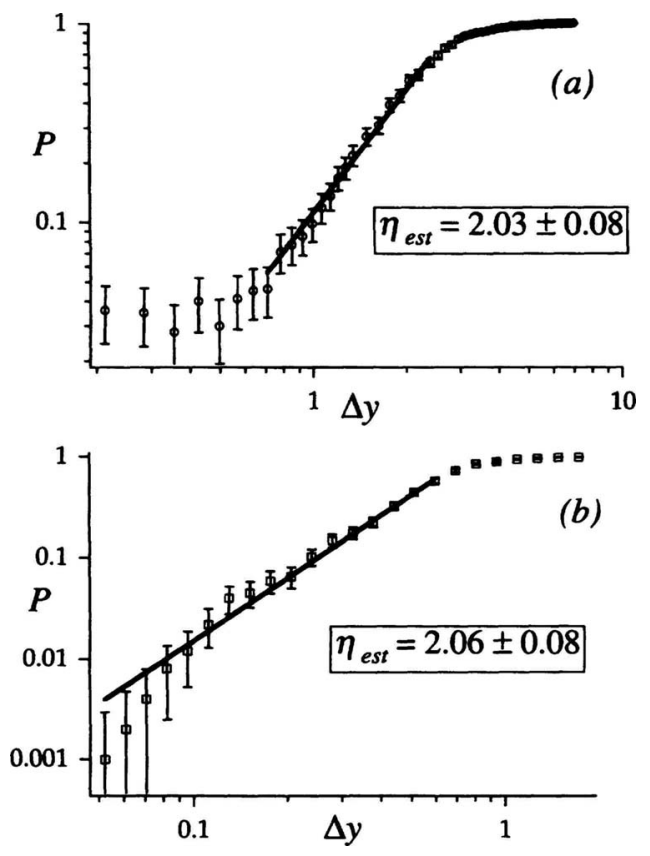

FIG. 31. Probability of going to the period-1 attractor as a function of the mode-2 Fourier amplitude $\Delta y$, obtained by (a) experiment and (b) numerical integration. From Heagy et al., 1994b.

most people ignore is that these structures represent examples of fractality in nature. In fact, these peculiar shapes were observed in several classical experiments (Yamada and Matsui, 1978; van Dyke, 1982) but they were not analyzed from the point of view of nonlinear dynamics, as the concept of fractality had not been yet sufficiently developed. When fractality in open hydrodynamical flows started to be studied from the point of view of chaotic scattering, that is, as the consequence of the existence of a chaotic saddle embedded in the system, it became clear that it would be a good context to develop experimental verifications of fractal basin boundaries. Péntek et al. (1995b) observed that determining approximately the chaotic saddle and its stable and unstable manifolds should be possible in a laboratory experiment. This was done as follows. Inject a droplet in the upstream region, just before the mixing region where the chaotic saddle is inserted. After several periods of time, the ink will have traced out the unstable manifold; [see Sec. V.B.2 and Tél and Gruiz (2006) for several examples of natural chaotic advection phenomena, where plankton, clouds, ice, etc., spread around different obstacles such as islands or mountains and give rise to beautiful and real fractal unstable manifolds]. Plotting the stable manifold is more difficult and needs video techniques (Solomon et al., 1993, 1994; Sommerer and Ott, 1993a; Sommerer, 1994), as we must find the points that approach the chaotic saddle and stay in its neighborhood for a long time. For the chaotic saddle, one needs to sprinkle a lot of orbits in the mixing region, keeping the trajectory of those that stay in the system for a long time. The chaotic saddle is obtained from a section in the middle of the process. Finally, Péntek et al. (1995b) presented a modification of the sprinkler method (Kantz and Grassberger, 1985; Lai et al., 1993) for real systems. The main advantage of this method is that it provides all three invariant sets at the same time. We fix a critical number $n_{0}$ of periods and follow several orbits that stay in the mixing region for more than $n_{0}$ periods. The initial points are the stable manifold, the final points are the unstable manifold, and the middle points are the chaotic saddle. The accuracy of the method increases with $n_{0}$.

Despite all these methods, very few real experiments have been done in this direction. An easy-to-read review of the applicability of chaos theory to the study of twodimensional fluid flows was done by Sommerer et al. (1997). They pay special attention to the different experimental realizations, and explain the advantages and disadvantages of approaching such a hard task with the tools of nonlinear dynamics (that is, chaotic advection). Furthermore, they explain the way to calculate Lyapunov exponents [by applying the particle tracking technique (Diamond, 1994)], the fractal dimension, and the average decay time of the fractal structures associated to a fluid experiment. These quantities are specially important when developing an experiment, as in Kantz and Grassberger (1985) a simple theoretical relation between them was obtained, and therefore the verification of this relation is a proof of the certainty of the experimental results.

Among the real visualizations of fractal structures shown in Sommerer et al. (1997), it is worth remarking on the first experimental evidence of chaotic scattering in a fluid wake [work presented by Sommerer et al. (1996)]. The experiment consisted of a moving hard cylinder in a canal. The results were a demonstration of the difficulty that experimentalists face when trying to obtain information about fractality in real systems. They were obliged to verify the existence of chaotic scattering in an indirect way, as obtaining the plot of a scattering function with singularities seems in practice impossible for a real fluid wake. Among other evidences, they observed in the experiment that a fraction of the initial conditions stay for a long time in the mixing region just after the cylinder. Furthermore, they created a structure that resembles fractal, whose dimension is indeed noninteger and coincides with the theoretically expected value Kantz and Grassberger (1985). Figure 32 shows the observed dye lines after the cylinder, and how they correspond precisely to the computed streak lines obtained using a multigrid domain decomposition approach incorporating the pseudospectral element method; see Ku et al. $(1989,1990,1994)$ for the details of this numerical method.

\section{Optical systems}

In 1999 the first real verification of the existence of Wada basins was carried out (Sweet et al., 1999). The experiment was an optical three-dimensional chaotic scattering billiard, and so simple to build that anyone can make it at home. Place four mirrored spheres (e.g., 


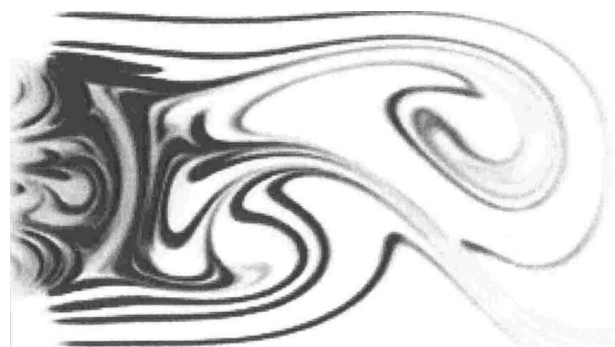

(a)

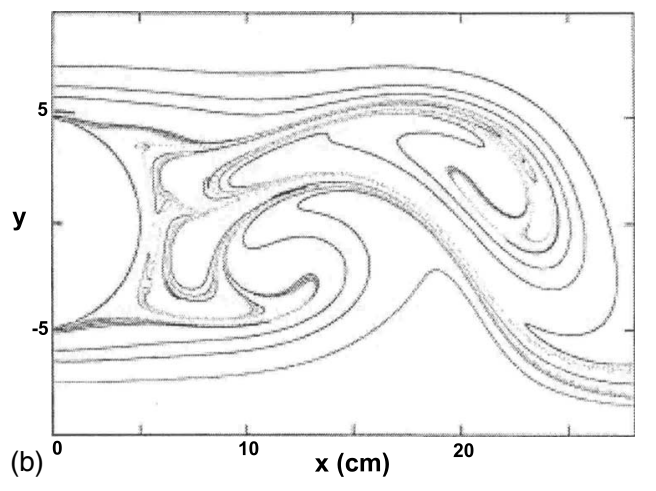

FIG. 32. Fractal structures can be detected in nature with the naked eye: (a) fractal dye lines obtained with a charge-coupled device camera fixed to a movable cylinder in a fluid wake; (b) computed streak lines corresponding to a numerical twodimensional approach of the same system. From Sommerer et al., 1996.

four Christmas balls) forming a tetrahedron in such a way that three of its four exits are covered with white, red, and blue cardboards. Illuminate each colored exit from the outside and look at it from the open side, and what you see is a fractal structure where the boundaries of all three colors are mixed on all scales, satisfying the Wada property. This surprising discovery deserved the cover of Nature magazine (Sweet et al., 1999), as well as the cover of Ott (2002); Fig. 33 shows an example of this phenomenon.

The work by Sweet et al. was the proof that the easiest way to verify the existence of fractal basins associated to chaotic scattering is the use of mirrored structures in optical systems. There are two reasons. First, optical configurations show the fundamental advantage of presenting in only one experiment the totality of the basin diagram, as each ray of light represents one orbit. Second, they are usually cheap and easy to manipulate, as only mirrors and a digital camera (or a charge-coupled device camera) are needed.

Sweet and Ott (2000a, 2000b) numerically studied a system based on a mirrored ellipsoid placed inside a hard tube of four curved walls. The system was thoroughly analyzed, and clear explanations were presented to build the system. Two exits were possible, as light could escape from the system in the upward and downward directions. Furthermore, one of the main results was that the basin boundaries obtained had the structure of a continuous, nowhere-differentiable surface that could be appreciated with the naked eye. However, the

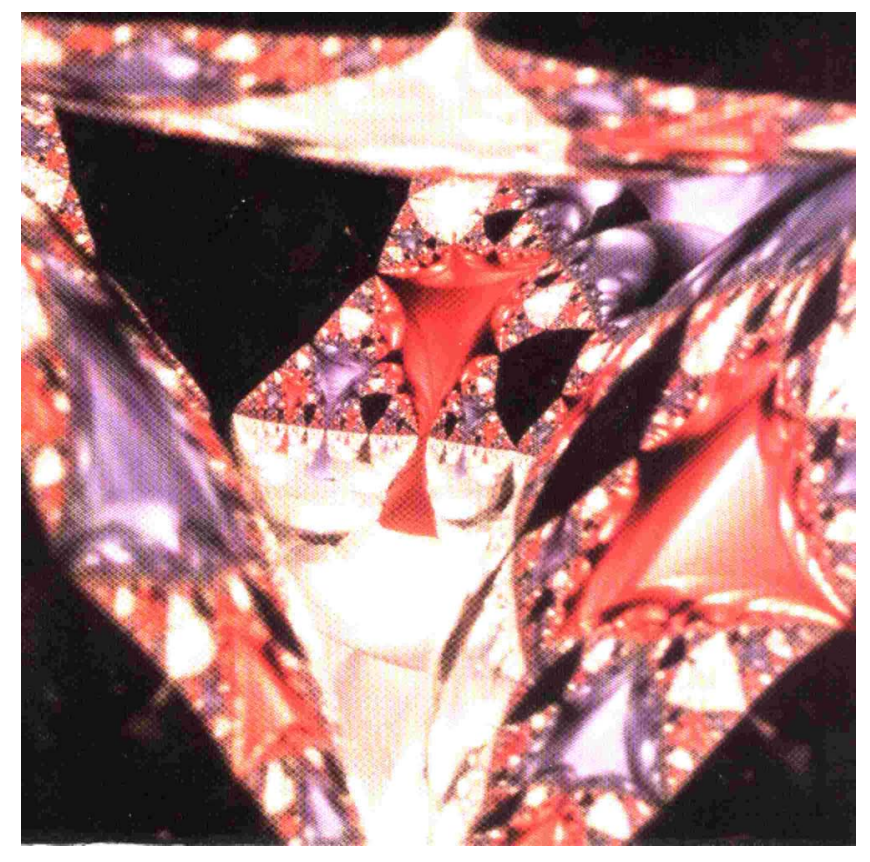

FIG. 33. (Color online) Experimental Wada basins obtained by placing four Christmas balls forming a tetrahedron and covering three of its four exits with red, blue, and white cardboard. Adapted from Ott, 2002.

experiment was not carried out, and we had to wait one year to see the first real manifestation of these peculiar basins. The same research group, working with a configuration of four mirrored spheres placed in a way that each one was in contact only with two other spheres and there were again two possible exits, showed in Sweet, Zeff, et al. (2001) real images of continuous but nowhere-differentiable basin boundaries, and compared their properties with those of the Wada basins present in the tetrahedron configuration already studied by Sweet et al. (1999) (where four exits are possible). The fractal dimension, as Sommerer et al. (1996) did for photos of fluids, was obtained from the digital images. The results matched the numerical predictions well. In order to encourage the reader to follow this line of research and take real images of new fractal basin boundaries in optical configurations, precise details of the experimental realization were given by Sweet, Zeff, et al. (2001). Furthermore, several possibilities for future study were proposed, such as placing four spheres in a tetrahedron configuration and lifting the upper one, or placing the spheres in a tetrahedron configuration in such a way that they are not in contact. This last possibility is especially challenging, as there is only one possible exit, and therefore there is only one basin defined. The existence of the stable manifold of the chaotic saddle should be detected by searching in the digital photograph for fractal regions where the image is darker (that is, the orbits that remain inside the system for a longer time, and are more absorbed by the spheres).

Finally, and as another example of possible future work, Aguirre and Sanjuán (2003) presented a numerical analysis of a peculiar phenomenon that takes place 
when the size of the exits of open Hamiltonian systems tends to zero. These fractal basins were named uncertain basins because of their dramatic consequences for predictability. The striking result was that, when the size of the exits shrinks, the exit basins show a behavior similar to that of riddled basins in dissipative systems; see Sec. VIII.A for more information on riddledlike basins. The exit basins suffer a total fractalization, tending to become their own boundaries while the dimension of the invariant sets tends to that of phase space. Furthermore, these invariant sets tend to fill up the whole phase space for both hyperbolic and nonhyperbolic systems, and an experimental verification of this fact seems a promising task. In the paper, an experimental optical verification of these results was proposed, based on a simple triangular configuration of three curved mirrors in which the distance between mirrors $d$ could be shrunk. If two of its three exits are covered with different colors (e.g., red and yellow cardboard) while we look into the system through the third exit, vertical lines of both colors will be mixed on all scales in the boundary that separates them. They believe it would be challenging to verify if in the limit of very small distance $(d \rightarrow 0)$ the mixture of both colors (e.g., orange) is recognizable.

In summary, the observation in nature of fractal basin boundaries is still in its first steps, mainly due to the difficulty of developing real experiments with nonattracting sets of orbits. There is no doubt that this is one of the most fruitful open fields in experimental nonlinear dynamics, where so much work still should be done.

\section{CONCLUSIONS}

The ideas of chaos and fractals developed since the last half of the 20th century have had a significant impact on a large variety of scientific disciplines, such as astrophysics, general relativity and cosmology, atomic and molecular dynamics, fluid dynamics, plasma physics, optics, etc. They have affected many other fields as well, which in principle are far away from physics, such as economy or ecology, which have also turned out to be fruitful environments where fractal structures appear naturally. As a consequence of the many different situations in which fractal structures appear in dynamical systems, fractal basin boundaries in particular deserved a unifying review, where theoretical, numerical, and graphical results as well as experimental setups could coexist in an organized structure.

The natural way to analyze our ability to predict the future of a deterministic system is by studying the basin diagram made out of the initial conditions that lead to each final state, be it a basin of attraction in the case of a dissipative system or an exit basin in the case of an open Hamiltonian system. Therefore our main topic has been the analysis of the existence and nature of the different kinds of fractal basin, both in models and in real physical systems. Special attention has been paid to the broad span of interests that potential readers from all fields of physics may have.
- We have presented in a simple and clarifying way the main properties of a fractal basin boundary, that is, its nature related to the existence of an invariant chaotic set and the typical way to measure its fractality: the fractal dimension. In parallel to the theoretical explanations, extensive numerical and graphical techniques have been presented to obtain from each system all the information that the tools of nonlinear dynamics can offer about its future behavior.

- From the pioneering work of McDonald et al. (1985), where the first classification of fractal basin boundaries was presented, many other different types of fractal basin have appeared in the literature. This wide variety of examples needed an illustrative presentation and organization, casting light on the similarities and differences between them. We started out presenting them in a comparative way, but later we paid special attention to the two types that have given rise to more extensive work: Wada and riddled basins.

- Our main goal is to show that fractal structures not only are mathematical artifacts appearing in certain dynamical systems, but can be applied to many different fields, in both physics and other scientific disciplines. From this point of view, a final analysis of the experimental verification of fractal structures in physical and natural systems was convenient. Moreover, this emerging and challenging topic is one of the open areas in which we believe that other researchers might find a promising subject to work on, by applying some of the fundamental ideas to their particular research field.

\section{ACKNOWLEDGMENTS}

We thank Professor James A. Yorke for his careful reading of the manuscript, as well as for the fruitful comments and advice that we received from him during the writing of this manuscript. R.L.V. would like to thank C. Grebogi, S. R. Lopes, and R. F. Pereira for useful discussions and valuable suggestions. This work was supported by the Spanish Ministry of Science and Technology under Project No. BFM2003-03081, by the Spanish Ministry of Education and Science under Project No. FIS2006-08525, and by CNPq and CAPES (Brazilian Government Agencies).

\section{REFERENCES}

Aguirre, J., and M. A. F. Sanjuán, 2002, Physica D 171, 41. Aguirre, J., and M. A. F. Sanjuán, 2003, Phys. Rev. E 67, 056201.

Aguirre, J., J. C. Vallejo, and M. A. F. Sanjuán, 2001, Phys. Rev. E 64, 066208.

Aguirre, J., J. C. Vallejo, and M. A. F. Sanjuán, 2003, Int. J. Mod. Phys. B 17, 4171.

Aguirregabiria, J. M., 1997, Phys. Lett. A 224, 234.

Alexander, J. C., I. Kan, J. A. Yorke, and Z. You, 1992, Int. J. Bifurcation Chaos Appl. Sci. Eng. 2, 795. 
Alligood, K. T., and T. Sauer, 1988, Commun. Math. Phys. 120, 105.

Alligood, K. T., T. D. Sauer, and J. A. Yorke, 2000, Chaos. An Introduction to Dynamical Systems (Springer, New York).

Alligood, K. T., and J. A. Yorke, 1992, Ergod. Theory Dyn. Syst. 12, 377.

Anderson, D. M., and D. L. Garrison, 1997, Limnol. Oceanogr. 42, 1009.

Aref, H., and S. Balachandar, 1986, Phys. Fluids 29, 3515.

Aref, H., S. W. Jones, S. Mofina, and I. Zawadzki, 1989, Physica D 37, 423.

Arístegui, J., P. Tett, A. Hernández-Guerra, G. Basterretxea, M. F. Montero, K. Wild, P. Sangrá, S. Hernández-León, M. Cantón, J. A. García-Braun, M. Pacheco, and E. D. Barton, 1997, Deep-Sea Res., Part I 44, 71.

Ashwin, P., 2005, in Dynamics of Coupled Map Lattices and of Related Spatially Extended Systems, edited by J.-R. Chazottes and B. Fernández, Lecture Notes in Physics Vol. 671 (Springer, Berlin).

Ashwin, P., and M. Breakspear, 2001, Phys. Lett. A 280, 139.

Ashwin, P., J. Buescu, and I. Stewart, 1994, Phys. Lett. A 193, 126.

Ashwin, P., J. Buescu, and I. Stewart, 1996, Nonlinearity 9, 703. Ashwin, P., X.-C. Fu, and J. R. Terry, 2002, Nonlinearity 15, 633.

Balescu, R., M. Vlad, and F. Spineanu, 1998, Phys. Rev. E 58, 951.

Barge, M., and R. Gillette, 1991, Contemp. Math. 117, 13.

Barnsley, M. F., 1993, Fractals Everywhere, 2nd ed. (Academic, Cambridge, MA).

Barton, E., et al., 1998, Prog. Oceanogr. 41, 455.

Bellido, F., and J. B. Ramírez-Malo, 2006, Int. J. Non-Linear Mech. 41, 860.

Berry, M. V., 1981, Eur. J. Phys. 2, 91.

Birkhoff, G. D., 1927, Acta Math. 50, 359.

Birkhoff, G. D., 1932, Bull. Soc. Math. France 60, 1.

Blanchard, P., 1984, Bull., New Ser., Am. Math. Soc. 11, 1.

Blazejczyk-Okolewska, B., J. Brindley, and T. Kapitaniak, 2000, Chaos, Solitons Fractals 11, 2511.

Bleher, S., C. Grebogi, and E. Ott, 1990, Physica D 46, 87.

Bleher, S., C. Grebogi, E. Ott, and R. Brown, 1988, Phys. Rev. A 38, 930

Boccaletti, S., J. Kurths, G. Osipov, D. L. Valladares, and C. Zhou, 2002, Phys. Rep. 366, 1.

Bollt, E., Y.-C. Lai, and C. Grebogi, 1997, Phys. Rev. Lett. 79, 3787.

Born, M., 1955, Phys. Bl. 11, 49.

Breban, R., and H. E. Nusse, 2005, Physica D 207, 52.

Breban, R., H. E. Nusse, and E. Ott, 2003, Phys. Lett. A 319, 79.

Breymann, W., Z. Kovács, and T. Tél, 1994, Phys. Rev. E 50, 1994.

Brolin, H., 1966, Ark. Mat. 6, 103.

Brouwer, L. E. J., 1910, Math. Ann. 68, 422.

Brú, A., S. Albertos, J. L. Subiza, J. L. García-Asenjo, and I. Brú, 2003, Biophys. J. 85, 2948.

Brú, A., J. M. Pastor, I. Fernaud, I. Brú, S. Melle, and C. Berenguer, 1998, Phys. Rev. Lett. 81, 4008.

Bruhn, B., and B. P. Koch, 1993, Int. J. Bifurcation Chaos Appl. Sci. Eng. 3, 999.

Budyansky, M., M. Uleysky, and S. Prants, 2004, Physica D 195, 369 .
Bunde, A., and S. Havlin, 1994, Fractals in Science (Springer, Berlin).

Bunimovich, L. A., and Y. G. Sinai, 1980, Commun. Math. Phys. 78, 479.

Burkholder, J. M., E. J. Noga, C. W. Hobbs, H. B. Glasgow, and S. A. Smith, 1992, Nature (London) 358, 407.

Burrough, P. A., 1981, Nature (London) 294, 240.

Cantor, G., 1881, Math. Ann. 21, 545.

Cartwright, M. L., and J. E. Littlewood, 1945, J. Lond. Math. Soc. 20, 180.

Cartwright, M. L., and J. E. Littlewood, 1951, Ann. Math. 54, 1.

Cayley, A., 1879, Quart. J. Pure Appl. Math. 16, 179.

Cazelles, B., 2001a, Chaos, Solitons Fractals 12, 301.

Cazelles, B., 2001b, Phys. Rev. E 64, 032901.

Chaiken, J., R. Chevray, M. Tabor, and Q. M. Tan, 1986, Proc.

R. Soc. London, Ser. A 408, 154.

Chen, J., J. L. Rexford, and Y. C. Lee, 1990, Geophys. Res. Lett. 17, 1049.

Chilver, L., 2006, Nonlinear Dyn. 43, 3.

Codd, G. A., 1995, Water Sci. Technol. 32, 149.

Contopoulos, G., 1990, Astron. Astrophys. 231, 41.

Contopoulos, G., H. E. Kandrup, and D. Kaufmann, 1993, Physica D 64, 310.

Contopoulos, G., and D. Kaufmann, 1992, Astron. Astrophys. 253, 379.

Crisanti, A., M. Falcioni, A. Vulpiani, and G. Paladin, 1991, Riv. Nuovo Cimento 14, 1.

Cross, S. S., A. J. G. McDonagh, T. J. Stephenson, D. W. K. Cotton, and J. C. Underwood, 1995, Am. J. Dermatopathol. 17, 374 .

Curry, J. H., L. Garnett, and D. Sullivan, 1983, Commun. Math. Phys. 91, 267.

Cusumano, J. P., and B. W. Kimble, 1995, Nonlinear Dyn. 8, 213.

Cvitanović, P., R. Artuso, R. Mainieri, G. Tanner, and G. Vattay, 2005, Chaos: Classical and Quantum (ChaosBook.org, Niels Bohr Institute, Copenhagen).

da Silva, E. C., I. L. Caldas, R. L. Viana, and M. A. F. Sanjuán, 2002, Phys. Plasmas 9, 4917.

Dawson, S., C. Grebogi, T. Sauer, and J. A. Yorke, 1994, Phys. Rev. Lett. 73, 1927.

de Laplace, P. S., 1814, Essai Philosophique sur les Probabilités (Veuve Courcier, Paris).

de Moura, A. P. S., and C. Grebogi, 2001, Phys. Rev. Lett. 86, 2778.

de Moura, A. P. S., and P. S. Letelier, 1999, Phys. Lett. A 256, 362.

de Moura, A. P. S., and P. S. Letelier, 2000a, Phys. Rev. E 61, 6506.

de Moura, A. P. S., and P. S. Letelier, 2000b, Phys. Lett. A 266, 309.

de Moura, A. P. S., and P. S. Letelier, 2000c, Phys. Rev. E 62, 4784.

Diamond, S. D., 1994, Johns Hopkins APL Tech. Dig. 15 (3), 188.

Ding, M., C. Grebogi, E. Ott, and J. A. Yorke, 1990, Phys. Rev. A 42, 7025 .

Ding, M., and W. Yang, 1996, Phys. Rev. E 54, 2489.

Dorfman, J. R., 1999, An Introduction to Chaos in NonEquilibrium Statistical Mechanics (Cambridge University Press, Cambridge, England).

Douady, A., 1983, Séminaire Bourbaki 1982/1983 No. 599, p. 
161.

Douady, A., and J. H. Hubbard, 1982, C. R. Acad. Sci. Paris 294, 123.

Dronov, V., and E. Ott, 2000, Chaos 10, 291.

Eckhardt, B., 1987, J. Phys. A 20, 5971.

Eckhardt, B., 1988, Physica D 33, 89.

Eckhardt, B., and C. Jung, 1986, J. Phys. A 19, L829.

Eckmann, J. P., 1983, Recherche 14, 260.

Eckmann, J. P., and D. Ruelle, 1985, Rev. Mod. Phys. 57, 617.

Edouard, S., B. Legras, F. Lefvre, and R. Eymard, 1996, Nature (London) 384, 444.

Edouard, S., B. Legras, and V. Zeitlin, 1996, J. Geophys. Res. 101, 16771.

Engelhardt, W., and W. Fenenberg, 1978, J. Nucl. Mater. 76-77, 518.

Eykholt, R., and D. K. Umberger, 1988, Physica D 30, 43.

Farmer, J. D., 1985, Phys. Rev. Lett. 55, 351.

Fatou, P., 1919, Bull. Soc. Math. France 47, 161.

Fatou, P., 1920, Bull. Soc. Math. France 48, 33.

Feudel, U., C. Grebogi, B. R. Hunt, and J. A. Yorke, 1996, Phys. Rev. E 54, 71.

Feynman, R. P., R. B. Leighton, and M. Sands, 1963, Mainly Mechanics, Radiation and Heat, The Feynman Lectures on Physics Vol. 1 (Addison-Wesley, Reading, MA).

Flaherty, J. E., and F. C. Hoppensteadt, 1978, Stud. Appl. Math. 58, 5.

Frame, M. L., and B. B. Mandelbrot, 2002, Fractals, Graphics, and Mathematics Education (Cambridge University Press, Cambridge, England).

Fujisaka, H., and T. Yamada, 1983, Prog. Theor. Phys. 69, 32.

Gaite, J., A. Domínguez, and J. Pérez-Mercader, 1999, Astrophys. J. Lett. 522, L5.

Gaite, J., and S. C. Manrubia, 2002, Mon. Not. R. Astron. Soc. 335, 977.

Gaspard, P., 2005, Chaos, Scattering, and Statistical Mechanics (Cambridge University Press, Cambridge, England).

Gaspard, P., and S. A. Rice, 1989a, J. Chem. Phys. 90, 2225.

Gaspard, P., and S. A. Rice, 1989b, J. Chem. Phys. 90, 2242.

Gaspard, P., and S. A. Rice, 1989c, J. Chem. Phys. 90, 2255.

Gause, G. F., and A. A. Witt, 1935, Am. Nat. 69, 596.

Gibson, C. H., and W. H. Thomas, 1995, J. Geophys. Res. 100, 24841.

Gilpin, M. E., 1975, Am. Nat. 109, 51.

Gollub, J. P., and D. J. Pine, 2006, Phys. Today 59 (8), 8.

Gong, P.-L., and J.-X. Xu, 2001, Phys. Rev. E 63, 031906.

Grassberger, P., 1986, in Chaos, edited by A. V. Holden (Manchester University, Manchester).

Grebogi, C., E. Kostelich, E. Ott, and J. A. Yorke, 1987, Physica D 25, 347.

Grebogi, C., S. W. McDonald, E. Ott, and J. A. Yorke, 1983, Phys. Lett. 99A, 415.

Grebogi, C., S. W. McDonald, E. Ott, and J. A. Yorke, 1985, Phys. Lett. 110A, 1.

Grebogi, C., H. E. Nusse, E. Ott, and J. A. Yorke, 1988, in Dynamical Systems, edited by J. C. Alexander, Springer Lecture Notes in Mathematics Vol. 1342 (Springer, New York), pp. 220-250.

Grebogi, C., E. Ott, and J. A. Yorke, 1982, Phys. Rev. Lett. 48, 1507.

Grebogi, C., E. Ott, and J. A. Yorke, 1983a, Physica D 7, 181. Grebogi, C., E. Ott, and J. A. Yorke, 1983b, Phys. Rev. Lett. 50, 935.

Grebogi, C., E. Ott, and J. A. Yorke, 1985, Ergod. Theory
Dyn. Syst. 5, 341.

Grebogi, C., E. Ott, and J. A. Yorke, 1987, Physica D 24, 243. Guckenheimer, J., and P. Holmes, 1983, Nonlinear Oscillations, Dynamical Systems, and Bifurcations in Vector Fields (Springer, New York).

Gulick, D., 1992, Encounters with Chaos (McGraw-Hill, New York).

Gumowsky, I., and C. Mira, 1980, Recurrences and Discrete Dynamical Systems, Lecture Notes in Mathematics Vol. 809 (Springer, Berlin).

Gwinn, E. G., and R. M. Westervelt, 1985, Phys. Rev. Lett. 54, 1613.

Gwinn, E. G., and R. M. Westervelt, 1986, Phys. Rev. A 33, 4143.

Hammel, S. M., and C. K. R. T. Jones, 1989, Physica D 35, 87. Hanson, J. D., 1987, Phys. Rev. A 35, 1470.

Hardin, G., 1960, Science 131, 1292.

Hasler, M., and Y. L. Maistrenko, 1997, IEEE Trans. Circuits Syst., I: Fundam. Theory Appl. 44, 856.

Hayli, A., and A. Vidovic, 1994, Phys. Lett. A 195, 59.

Heagy, J. F., T. L. Carroll, and L. M. Pecora, 1994a, Phys. Rev. E 50, 1874.

Heagy, J. F., T. L. Carroll, and L. M. Pecora, 1994b, Phys. Rev. Lett. 73, 3528.

Hénon, M., 1976, Commun. Math. Phys. 50, 69.

Hénon, M., and C. Heiles, 1964, Astron. J. 69, 73.

Hocking, J. G., and G. S. Young, 1961, Topology (AddisonWesley, Reading, MA).

Hofbauer, F., J. Hofbauer, P. Raith, and T. Steinberger, 2004, J. Math. Biol. 49, 293.

Holmes, P., 1990, Phys. Rep. 193, 137.

Horvath, G. Zs. K., J.-L. Hernandez-Pozos, K. Dholakia, J. Rink, D. M. Segal, and R. C. Thompson, 1998, Phys. Rev. A 57, 1944.

Hsu, G.-H., E. Ott, and C. Grebogi, 1988, Phys. Lett. A 127, 199.

Huisman, J., and F. J. Weissing, 1999, Nature (London) 402 , 407.

Huisman, J., and F. J. Weissing, 2001, Am. Nat. 157, 488.

Hunt, B. R., E. Ott, and E. Rosa, Jr., 1999, Phys. Rev. Lett. 82, 3597.

Hunt, B. R., and J. A. Yorke, 1993, Nonlinear Sci. Today 3, 1. Jakobson, M. V., 1968, Math. USSR. Sb. 6, 97.

Jánosi, I. M., and T. Tél, 1994, Phys. Rev. E 49, 2756.

Jones, B. J. T., V. J. Martínez, E. Saar, and V. Trimble, 2004, Rev. Mod. Phys. 76, 1211.

Julia, G., 1918, J. Math. Pures Appl. 4, 47.

Jung, C., and H. J. Scholz, 1987, J. Phys. A 20, 3607.

Jung, C., and T. Tél, 1991, J. Phys. A 24, 2793.

Jung, C., T. Tél, and E. Ziemniak, 1993, Chaos 3, 555.

Kan, I., 1994, Bull., New Ser., Am. Math. Soc. 31, 68.

Kantz, H., and P. Grassberger, 1985, Physica D 17, 75.

Kapitaniak, T., 2001, Chaos, Solitons Fractals 12, 2363.

Kapitaniak, T., and Y. L. Maistrenko, 1998, Chaos, Solitons Fractals 9, 271.

Kapitaniak, T., and Y. L. Maistrenko, 1999, Physica D 126, 18. Kapitaniak, T., Y. L. Maistrenko, and C. Grebogi, 2003, Chaos, Solitons Fractals 17, 61.

Kapitaniak, T., Yu. Maistrenko, A. Stefanski, and J. Brindley, 1998, Phys. Rev. E 57, R6253.

Kaplan, J. L., and J. A. Yorke, 1979, Commun. Math. Phys. 67, 93.

Károlyi, G., A. Péntek, I. Scheuring, T. Tél, and Z. Toroczkai, 
2000, Proc. Natl. Acad. Sci. U.S.A. 97, 13661.

Károlyi, G., A. Péntek, Z. Toroczkai, T. Tél, and C. Grebogi, 1999, Phys. Rev. E 59, 5468.

Katz, A. J., and A. H. Thompson, 1985, Phys. Rev. Lett. 54, 1325.

Keck, J. C., 1967, Adv. Chem. Phys. 13, 85.

Kennedy, J., 1995, A Brief History of Indecomposable Continua. Continua with the Houston Problem Book, Lecture Notes in Pure and Applied Mathematics, Vol. 170 (CRC, New York), pp. 103-126.

Kennedy, J., M. A. F. Sanjuán, J. A. Yorke, and C. Grebogi, 1999, Topol. Appl. 94, 207.

Kennedy, J., and J. A. Yorke, 1991, Physica D 51, 213.

Kennedy, J., and J. A. Yorke, 1997, Topol. Appl. 80, 201.

Kim, S.-Y., and W. Lim, 2001, Phys. Rev. E 63, 026217.

Klages, R., and C. Dellago, 2000, J. Stat. Phys. 101, 145.

Kohmoto, M., L. P. Kadanoff, and C. Tang, 1983, Phys. Rev. Lett. 50, 1870.

Koon, W. S., M. W. Lo, J. E. Marsden, and S. D. Ross, 2000, Chaos 10, 427.

Kostelich, E. J., I. Kan, C. Grebogi, E. Ott, and J. A. Yorke, 1997, Physica D 109, 81.

Kóvacs, Z., and L. Wiesenfeld, 1995, Phys. Rev. E 51, 5476.

Kóvacs, Z., and L. Wiesenfeld, 2001, Phys. Rev. E 63, 056207.

Ku, H.-C., H. E. Gilreath, R. Raul, and J. C. Sommerer, 1994, Contemp. Math. 180, 509.

Ku, H.-C., R. S. Hirsh, T. D. Taylor, and A. P. Rosenburg, 1989, J. Comput. Phys. 83, 260.

Ku, H.-C., A. P. Rosenburg, and T. D. Taylor, 1990, Lecture Notes in Physics, edited by K. W. Morton (Springer, Berlin).

Kuratowski, C., 1924, Fundam. Math. 6, 130.

Lai, Y.-C., 1995, Phys. Rev. E 51, 2902.

Lai, Y.-C., 2000, Phys. Rev. E 62, R4505.

Lai, Y.-C., 2001, Physica D 150, 1.

Lai, Y.-C., and V. Andrade, 2001, Phys. Rev. E 64, 056228.

Lai, Y.-C., A. P. S. de Moura, and C. Grebogi, 2000, Phys. Rev. E 62, 6421.

Lai, Y.-C., and C. Grebogi, 1995, Phys. Rev. E 52, R3313.

Lai, Y.-C., and C. Grebogi, 1996, Phys. Rev. Lett. 77, 5047.

Lai, Y.-C., and C. Grebogi, 1999, Phys. Rev. Lett. 83, 2926.

Lai, Y.-C., and C. Grebogi, 2000, Phys. Rev. Lett. 85, 473.

Lai, Y.-C., C. Grebogi, J. A. Yorke, and S. C. Venkataramani, 1996, Phys. Rev. Lett. 77, 55.

Lai, Y.-C., T. Tél, and C. Grebogi, 1993, Phys. Rev. E 48, 709.

Lai, Y.-C., and R. L. Winslow, 1994, Phys. Rev. Lett. 72, 1640.

Lau, Y. T., J. M. Finn, and E. Ott, 1991, Phys. Rev. Lett. 66, 978.

Levi, M., 1981, Mem. Am. Math. Soc. 32, 1.

Levinson, N., 1949, Ann. Math. 50, 127.

Li, T. Y., and J. A. Yorke, 1975, Am. Math. Monthly 82, 985.

Lorentz, H. A., 1905a, Proc. R. Acad. Sci. Amsterdam 7, 438.

Lorentz, H. A., 1905b, Proc. R. Acad. Sci. Amsterdam 7, 585.

Lorentz, H. A., 1905c, Proc. R. Acad. Sci. Amsterdam 7, 684.

Lorenz, E., 1963, J. Atmos. Sci. 20, 130.

Lorenz, H.-W., and H. E. Nusse, 2002, Chaos, Solitons Fractals $13,957$.

Losa, G. A., 1995, Pathologica 87, 310.

Losa, G. A., G. Baumann, and T. F. Nonnenmacher, 1992, Pathol. Res. Pract. 188, 680.

Maistrenko, Yu. L., V. L. Maistrenko, A. Popovich, and E. Mosekilde, 1998, Phys. Rev. Lett. 80, 1638.

Maistrenko, Yu. L., V. L. Maistrenko, O. Popovich, and E. Mosekilde, 1999a, Phys. Lett. A 262, 355.
Maistrenko, Yu. L., V. L. Maistrenko, O. Popovych, and E. Mosekilde, 1999b, Phys. Rev. E 60, 2817.

Mandelbrot, B. B., 1967, Science 156, 636.

Mandelbrot, B. B., 1975, Les Objets Fractals, Forme, Hasard et Dimension (Flammarion, Paris).

Mandelbrot, B. B., 1977, Fractals, Form, Chance, and Dimension (Freeman, San Francisco).

Mandelbrot, B. B., 1980, Ann. N.Y. Acad. Sci. 357, 249.

Mandelbrot, B. B., 1982, The Fractal Geometry of Nature (Freeman, San Francisco).

Mané, R., P. Sad, and D. Sullivan, 1983, Ann. Sci. Ec. Normale Super. 16, 193.

Manscher, M., M. Nordahn, E. Mosekilde, and Y. L. Maistrenko, 1998, Phys. Lett. A 238, 358.

Marcos, S. H. C., S. R. Lopes, and R. L. Viana, 2003, Chaos, Solitons Fractals 15, 417.

May, R. M., and W. J. Leonard, 1975, SIAM J. Appl. Math. 29, 243.

McDonald, S. W., C. Grebogi, E. Ott, and J. A. Yorke, 1985, Physica D 17, 125.

Metcalfe, G., and J. M. Ottino, 1994, Phys. Rev. Lett. 72, 2875. Ottino, J. M., A. Souvaliotis, and G. Metcalfe, 1995, Chaos, Solitons Fractals 6, 425.

Milnor, J., 1985, Commun. Math. Phys. 99, 177.

Moon, F., and P. J. Holmes, 1979, J. Sound Vib. 65, 275.

Moon, F., and G.-X. Li, 1985, Phys. Rev. Lett. 55, 1439.

Moresco, P., and S. P. Dawson, 1999, Physica D 126, 38.

Motter, A. E., and Y.-C. Lai, 2001, Phys. Rev. E 65, 015205(R).

Motter, A. E., and P. S. Letelier, 2001a, Regular Chaotic Dyn. 6, 215.

Motter, A. E., and P. S. Letelier, 2001b, URL http:// www.citebase.org/cgi-bin/citations?id=oai:arXiv.org:nlin/ 0101021.

Motter, A. E., and P. S. Letelier, 2001c, Phys. Lett. A 285, 127.

Muzzio, F. J., P. D. Swanson, and J. M. Ottino, 1992, Int. J. Bifurcation Chaos Appl. Sci. Eng. 2, 37.

Nakajima, H., and Y. Ueda, 1996, Physica D 99, 35.

Napiórkowski, M., 1986, Phys. Rev. A 33, 4423.

Nauenberg, M., and H. J. Schellnhuber, 1989, Phys. Rev. Lett. 62, 1807.

Nobbe, B., 1995, J. Stat. Phys. 78, 1591.

Nusse, H. E., and J. A. Yorke, 1989, Physica D 36, 137.

Nusse, H. E., and J. A. Yorke, 1991, Nonlinearity 4, 1183.

Nusse, H. E., and J. A. Yorke, 1996, Physica D 90, 242.

Nusse, H. E., and J. A. Yorke, 1997, Dynamics: Numerical Explorations (Springer, New York).

Nusse, H. E., and J. A. Yorke, 2000, Phys. Rev. Lett. 84, 626.

Ostlund, S., R. Pandit, D. Rand, H. J. Schellnhuber, and E. D.

Siggia, 1983, Phys. Rev. Lett. 50, 1873.

Ott, E., 1981, Rev. Mod. Phys. 53, 655.

Ott, E., 2002, Chaos in Dynamical Systems (Cambridge University Press, Cambridge).

Ott, E., J. C. Alexander, I. Kan, J. C. Sommerer, and J. A. Yorke, 1994, Physica D 76, 384.

Ott, E., and J. C. Sommerer, 1994, Phys. Lett. A 188, 39.

Ott, E., J. C. Sommerer, J. C. Alexander, I. Kan, and J. A. Yorke, 1993, Phys. Rev. Lett. 71, 4134.

Ott, E., and T. Tél, 1993, Chaos 3, 417.

Ott, E., E. D. Yorke, and J. A. Yorke, 1985, Physica D 16, 62. Ottino, J. M., 1989, The Kinematics of Mixing: Stretching, Chaos and Transport (Cambridge University Press, Cambridge, England).

Ottino, J. M., 1990, Annu. Rev. Fluid Mech. 22, 207. 
Park, B.-S., C. Grebogi, E. Ott, and J. A. Yorke, 1989, Phys. Rev. A 40, 1576.

Parmenter, R. H., and L. Y. Yu, 1994, Phys. Lett. A 189, 181.

Pecora, L. M., and T. L. Carroll, 1990, Phys. Rev. Lett. 64, 821.

Pecora, L. M., T. L. Carroll, G. A. Johnson, D. J. Mar, and J. F. Heagy, 1997, Chaos 7, 520.

Peitgen, H. O., H. Jürgens, and D. Saupe, 1992, Chaos and Fractals. New Frontiers of Science (Springer, Berlin).

Peitgen, H. O., and P. H. Richter, 1986, The Beauty of Fractals (Springer, New York).

Peitgen, H. O., D. Saupe, and F. V. Haesler, 1984, Math. Intell. 6, 11.

Pelikan, S., 1985, Trans. Am. Math. Soc. 292, 692.

Péntek, A., T. Tél, and Z. Toroczkai, 1995a, J. Phys. A 28, 2191.

Péntek, A., T. Tél, and Z. Toroczkai, 1995b, Fractals 3, 33.

Péntek, A., T. Tél, and Z. Toroczkai, 1996, Int. J. Bifurcation Chaos Appl. Sci. Eng. 62619.

Péntek, A., Z. Toroczkai, T. Tél, C. Grebogi, and J. A. Yorke, 1995, Phys. Rev. E 51, 4076.

Pereira, R. F., S. Camargo, S. E. de S. Pinto, S. R. Lopes, and R. L. Viana, 2008, Phys. Rev. E 78, 056214.

Pereira, R. F., S. E. de S. Pinto, R. L. Viana, S. R. Lopes, and C. Grebogi, 2007, Chaos 17, 023131.

Pikovsky, A., and P. Grassberger, 1991, J. Phys. A 24, 4587.

Pikovsky, A., M. Rosenblum, and J. Kurths, 2003, Synchronization: A Universal Concept in Nonlinear Sciences (Cambridge University Press, Cambridge, England).

Pine, D. J., J. P. Gollub, J. F. Brady, and A. M. Leshansky, 2005, Nature (London) 438, 997.

Poincaré, H., 1885, Acta Math. 7, 259.

Poincaré, H., 1897, Les Méthodes Nouvelles de la Mécanique Céleste (Gauthier-Villars, Paris), Vols. I and II.

Poincaré, H., 1908, Science et Méthode (Flammarion, Paris).

Poon, L., J. Campos, E. Ott, and C. Grebogi, 1996, Int. J. Bifurcation Chaos Appl. Sci. Eng. 6, 251.

Portela, J. S. E., I. L. Caldas, R. L. Viana, and M. A. F. Sanjuán, 2007, Int. J. Bifurcation Chaos Appl. Sci. Eng. 17, 4067.

Prigogine, I., 1996, La Fin des Certitudes (Editions Odile Jacob, Paris).

Rammal, R., 1984, J. Phys. (France) 45, 191.

Rosa, E., and E. Ott, 1999, Phys. Rev. E 59, 343.

Rosa, E., E. Ott, and M. H. Hess, 1998, Phys. Rev. Lett. 80, 1642.

Sander, L. M., 1986, Nature (London) 322, 789.

Sanjuán, M. A. F., T. Horita, and K. Aihara, 2003, Chaos 13, 17.

Sanjuán, M. A. F., J. Kennedy, C. Grebogi, and J. A. Yorke, 1997, Chaos 7, 125.

Sanjuán, M. A. F., J. Kennedy, E. Ott, and J. A. Yorke, 1997, Phys. Rev. Lett. 78, 1892.

Scheuring, I., G. Károly, A. Péntek, T. Tél, and Z. Toroczkai, 2000, Freshwater Biol. 45, 123.

Schmick, M., E. Goles, and M. Markus, 2000, Phys. Rev. E 62, 397.

Schneider, J., T. Tél, and Z. Neufeld, 2002, Phys. Rev. E 66, 066218.

Schwartz, I. B., and T. W. Carr, 1999, Phys. Rev. E 59, 6658.

Seoane, J. M., J. Aguirre, M. A. F. Sanjuán, and Y.-C. Lai, 2006, Chaos 16, 023101.

Seoane, J. M., and M. A. F. Sanjuán, 2008, Phys. Lett. A 372, 110.

Seoane, J. M., M. A. F. Sanjuán, and Y.-C. Lai, 2007, Phys.
Rev. E 76, 016208.

Simó, C., 1999, in Hamiltonian Systems with Three or More Degress of Freedom, edited by C. Simó (Kleuwer Academic, Dordrecht), pp. 223-241.

Siopis, C. V., H. E. Kandrup, and G. Contopoulos, 1997, Celest. Mech. Dyn. Astron. 65, 57.

Smale, S., 1967, Bull. Am. Math. Soc. 73, 747.

Smale, S., 1976, J. Math. Biol. 3, 5.

Smilansky, U., 1992, in The Classical and Quantum Theory of Chaotic Scattering, in Chaos and Quantum Physics, edited by M.-J. Giannoni, A. Voros, and J. Zinn-Justin (Elsevier, Amsterdam).

Solomon, T. H., E. R. Weeks, and H. L. Swinney, 1993, Phys. Rev. Lett. 71, 3975.

Solomon, T. H., E. R. Weeks, and H. L. Swinney, 1994, Physica D 76, 70.

Sommerer, J. C., 1994, Physica D 76, 85.

Sommerer, J. C., 1995, Johns Hopkins APL Tech. Dig. 16 (4), 333.

Sommerer, J. C., H.-C. Ku, and H. E. Gilreath, 1996, Phys. Rev. Lett. 77, 5055.

Sommerer, J. C., and E. Ott, 1993a, Science 259, 335.

Sommerer, J. C., and E. Ott, 1993b, Nature (London) 365, 138.

Sommerer, J. C., E. Ott, and T. Tél, 1997, Johns Hopkins APL Tech. Dig. 18 (2), 193.

Steklain, A. F., and P. S. Letelier, 2006, Phys. Lett. A 352, 398.

Strogatz, S. H., 1994, Nonlinear Dynamics and Chaos (Perseus Books, Cambridge, MA).

Suetani, H., and T. Horita, 2001, Chaos 11, 795.

Sullivan, D., 1982, C. R. Acad. Sci. (Paris) 294, 301.

Sweet, D., H. E. Nusse, and J. A. Yorke, 2001, Phys. Rev. Lett. 86, 2261.

Sweet, D., and E. Ott, 2000a, Phys. Lett. A 266, 134.

Sweet, D., and E. Ott, 2000b, Physica D 139, 1.

Sweet, D., E. Ott, and J. A. Yorke, 1999, Nature (London) 399, 315.

Sweet, D., B. W. Zeff, E. Ott, and D. P. Lathrop, 2001, Physica D 154, 207.

Tan, N., Y. Chen, and J. Xu, 2003, Chaos, Solitons Fractals 18, 17.

Tél, T., A. P. S. de Moura, C. Grebogi, and G. Károlyi, 2005, Phys. Rep. 413, 91.

Tél, T., A. Fulop, and T. Vicsek, 1989, Physica A 159, 155.

Tél, T., and M. Gruiz, 2006, Chaotic Dynamics (Cambridge University Press, Cambridge, England).

Terry, J. R., and P. Ashwin, 2000, Phys. Rev. Lett. 85, 472.

Thomas, W. H., C. Tynan, and C. Gibson, 1997, in Progress in Phycological Research, edited by F. E. Round and D. J. Chapman (Biopress, Bristol), Vol. 12, p. 283.

Thompson, J. M. T., 1992, Physica D 58, 260.

Thompson, J. M. T., and H. B. Stewart, 2002, Nonlinear Dynamics and Chaos, Geometrical Methods for Engineers and Scientists, 2nd ed. (Wiley, Chichester).

Toroczkai, Z., G. Károlyi, A. Péntek, T. Tél, and C. Grebogi, 1998, Phys. Rev. Lett. 80, 500.

Toroczkai, Z., G. Károlyi, A. Péntek, T. Tél, C. Grebogi, and J. A. Yorke, 1997, Physica A 239, 235.

Troll, G., 1996, Chaos, Solitons Fractals 7, 1929.

Truhlar, D. G., B. C. Garrett, and S. J. Klippenstein, 1996, J. Phys. Chem. 100, 12771.

Umberger, D. K., and J. D. Farmer, 1985, Phys. Rev. Lett. 55, 661.

Uzer, T., C. Jaffé, J. Palacián, P. Yanguas, and S. Wiggins, 2002, 
Nonlinearity 15, 957.

Vandermeer, J., 2004, Ecol. Modell. 176, 65.

Vandermeer, J., L. Stone, and B. Blasius, 2001, Chaos, Solitons Fractals 12, 265.

van der Pol, B., 1920, Radio Rev. 1, 704.

van der Pol, B., 1927, Philos. Mag. 3, 65.

van Dyke, M., 1982, An Album of Fluid Motion (The Parabolic Press, Stanford, CA).

Varvoglis, H., K. Katsonis, M. Savopulos, and G. Maynard, 2001, Phys. Scr. 63, 272.

Veselý, K., and J. Podolský, 2000, Phys. Lett. A 271, 368.

Viana, R. L., S. E. de S. Pinto, J. R. R. Barbosa, and C. Grebogi, 2003, Int. J. Bifurcation Chaos Appl. Sci. Eng. 13, 3235.

Viana, R. L., and C. Grebogi, 2001, Int. J. Bifurcation Chaos Appl. Sci. Eng. 11, 2689.

Viana, R. L., C. Grebogi, S. E. de S. Pinto, S. R. Lopes, A. M. Batista, and J. Kurths, 2003, Phys. Rev. E 68, 067204.

Viana, R. L., C. Grebogi, S. E. de S. Pinto, S. R. Lopes, A. M. Batista, and J. Kurths, 2005, Physica D 206, 94.

Virgin, L., 2000, Introduction to Experimental Nonlinear Dynamics (Cambridge University Press, Cambridge, England).

Vulović, V. Z., and R. E. Prange, 1986, Phys. Rev. A 33, 576.

Waalkens, H., A. Burbanks, and S. Wiggins, 2004a, J. Chem. Phys. 121, 6207.

Waalkens, H., A. Burbanks, and S. Wiggins, 2004b, J. Phys. A 37, L257.

Wagner, F., and U. Stroh, 1993, Plasma Phys. Controlled Fusion 35, 1321.

Wallace, A. D., 1955, Bull. Am. Math. Soc. 61, 95.

Wiggins, S., 1990, Introduction to Applied Dynamical Systems and Chaos (Springer, Berlin).
Wiggins, S., 1992, Chaotic Transport in Dynamical Systems (Springer, Berlin).

Wiggins, S., 1994, Normally Hyperbolic Invariant Manifolds in Dynamical Systems (Springer, New York).

Wiggins, S., L. Wiesenfeld, C. Jaffé, and T. Uzer, 2001, Phys. Rev. Lett. 86, 5478.

Williams, R. F., 1967, Topology 6, 473.

Williams, R. F., 1974, Publ. Math., Inst. Hautes Etud. Sci. 43, 169.

Williams, R. F., 1979, Publ. Math., Inst. Hautes Etud. Sci. 50, 101.

Woltering, M., and M. Markus, 1999, Phys. Lett. A 260, 453.

Woltering, M., and M. Markus, 2000a, Chem. Phys. Lett. 321, 473.

Woltering, M., and M. Markus, 2000b, Phys. Rev. Lett. 84, 630.

Wooton, A. J., B. Carreras, H. Matsumoto, K. McGuire, W. A. Peebles, C. P. Ritz, P. W. Terry, and S. J. Sweben, 1990, Phys. Fluids B 2, 2879.

Yamada, H., and T. Matsui, 1978, Phys. Fluids 21, 292.

Yamamoto, T., and K. Kaneko, 1998, Prog. Theor. Phys. 100, 1089.

Yang, H. L., 2000, Phys. Rev. E 62, R4509.

Yang, H. L., and A. S. Pikovsky, 1999, Phys. Rev. E 60, 5474.

Yoneyama, K., 1917, Tohoku Math. J. 11-12, 43.

Yorke, J. A., and E. D. Yorke, 1979, J. Stat. Phys. 21, 263.

You, Z., E. J. Kostelich, and J. A. Yorke, 1991, Int. J. Bifurcation Chaos Appl. Sci. Eng. 1, 605.

Yousefi, S., Y. Maistrenko, and S. Popovich, 2000, Discrete Dyn. Nat. Soc. 5, 161. 